\title{
The luminous host galaxy, faint supernova and rapid afterglow rebrightening of GRB 100418A
}

\author{
A. de Ugarte Postigo ${ }^{1,2}$, C. C. Thöne ${ }^{1}$, K. Bensch ${ }^{1}$, A. J. van der Horst ${ }^{3,4}$, D. A. Kann ${ }^{1}$, Z. Cano ${ }^{1}$, L. Izzo ${ }^{1}$,
} P. Goldoni ${ }^{5}$, S. Martín ${ }^{6,7}$, R. Filgas ${ }^{8}$, P. Schady ${ }^{9}$, J. Gorosabel ${ }^{10,11,1, \dagger}$, I. Bikmaev ${ }^{12,13}$, M. Bremer ${ }^{14}$, R. Burenin ${ }^{15,16}$, A. J. Castro-Tirado ${ }^{1}$, S. Covino ${ }^{17}$, J. P. U. Fynbo ${ }^{2,18}$, D. Garcia-Appadoo ${ }^{6}$, I. de Gregorio-Monsalvo ${ }^{6,7}$, M. Jelínek ${ }^{19}$,

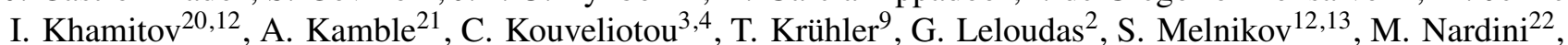
D. A. Perley ${ }^{23}$, G. Petitpas ${ }^{24}$, G. Pooley ${ }^{25}$, A. Rau ${ }^{9}$, E. Rol ${ }^{26,27}$, R. Sánchez-Ramírez ${ }^{28,1}$, R. L. C. Starling ${ }^{29}$, N. R. Tanvir ${ }^{29}$, K. Wiersema ${ }^{30}$, R. A. M. J. Wijers ${ }^{31}$, and T. Zafar ${ }^{32}$

(Affiliations can be found after the references)

Received 13 June 2018 / Accepted 24 July 2018

\begin{abstract}
Context. Long gamma-ray bursts (GRBs) give us the chance to study both their extreme physics and the star-forming galaxies in which they form. Aims. GRB 100418A, at a redshift of $z=0.6239$, had a bright optical and radio afterglow, and a luminous star-forming host galaxy. This allowed us to study the radiation of the explosion as well as the interstellar medium of the host both in absorption and emission.

Methods. We collected photometric data from radio to X-ray wavelengths to study the evolution of the afterglow and the contribution of a possible supernova (SN) and three $\mathrm{X}$-shooter spectra obtained during the first $60 \mathrm{~h}$.

Results. The light curve shows a very fast optical rebrightening, with an amplitude of $\sim 3$ magnitudes, starting $2.4 \mathrm{~h}$ after the GRB onset. This cannot be explained by a standard external shock model and requires other contributions, such as late central-engine activity. Two weeks after the burst we detect an excess in the light curve consistent with a SN with peak absolute magnitude $M_{V}=-18.5$ mag, among the faintest GRBSNe detected to date. The host galaxy shows two components in emission, with velocities differing by $130 \mathrm{~km} \mathrm{~s}^{-1}$, but otherwise having similar properties. While some absorption and emission components coincide, the absorbing gas spans much higher velocities, indicating the presence of gas beyond the star-forming regions. The host has a star formation rate of $\mathrm{SFR}=12.2 M_{\odot} \mathrm{yr}^{-1}$, a metallicity of $12+\log (\mathrm{O} / \mathrm{H})=8.55$, and a mass of $1.6 \times 10^{9} M_{\odot}$

Conclusions. GRB 100418A is a member of a class of afterglow light curves which show a steep rebrightening in the optical during the first day, which cannot be explained by traditional models. Its very faint associated SN shows that GRB-SNe can have a larger dispersion in luminosities than previously seen. Furthermore, we have obtained a complete view of the host of GRB 100418A owing to its spectrum, which contains a remarkable number of both emission and absorption lines.
\end{abstract}

Key words. gamma-ray burst: individual: GRB 100418A - supernovae: individual: GRB 100418A - galaxies: dwarf - ISM: abundances ISM: kinematics and dynamics

\section{Introduction}

Long gamma-ray bursts (GRBs) are produced during the dramatic death of very massive stars in which the core collapses, forming a black hole, while material is ejected through narrow polar jets (Rhoads 1997). In order to see the prominent gamma-ray emission, the observer's perspective needs to be within the opening angle of the jet. In the most accepted model (Piran 1999), the gamma rays are produced during internal collisions of material moving at ultrarelativistic speeds within the jets. When the ejecta collide with the material that surrounds the star, they are decelerated and at the same time intense synchrotron radiation is emitted. This radiation can be observed at all wavelengths between radio and X-rays, and is known as the forward shock. This electromagnetic emission is often observed for several days before it fades away in optical and X-rays, but can occasionally be followed

* This work makes use of data obtained at the following telescopes/observatories: VLT/Paranal (proposals 085.A-0009, 085.D0773), GTC/ORM (proposals GTC74-10A, GTCMULTIPLE2B17A), Keck/MK, Subaru/MK, 3.5m/CAHA, UKIRT/MK, WHT/ORM, RTT150/TUBITAK, Spitzer, PdBI/IRAM, WSRT/RO, Ryle/MRAO, and SMA/MK.

$\dagger$ Deceased. in radio for periods of weeks, months, or, in some exceptional cases, years. This synchrotron radiation is characterised by several power laws connected at characteristic frequencies (Sari et al. 1998). By studying their evolution we can determine some of the micro- and macro-physical parameters that drive the explosion. Occasionally the GRB afterglow can also have a reverse-shock component (Mészáros \& Rees 1999; Nakar \& Piran 2004), which originates within the shocked material that bounces back into the jet after the encounter of the forward shock with the circumstellar material. This reverse shock can be quite luminous but is typically shorter lived than the forward shock.

In this basic afterglow model, the light curves are also expected to evolve according to simple broken power laws. However, when sufficient sampling is available, the light curves often deviate from a simple evolution, showing flares, bumps, and wiggles (e.g. Jakobsson et al. 2004; de Ugarte Postigo et al. 2005) that require the addition of further parameters into the models. Energy injections either due to late central engine activity (Dai \& Lu 1998; Zhang \& Mészáros 2002) or to ejecta simultaneously emitted at different velocities (Rees \& Mészáros 1998; Sari \& Mészáros 2000), structured jets (Mészáros et al. 1998), patchy shells (Nakar \& Oren 2004), density fluctuations 
(Wang \& Loeb 2000; Ramirez-Ruiz et al. 2001), or double jets (Berger et al. 2003; Filgas et al. 2011; Kann et al. 2018) are some examples of models that try to explain some of the deviations from the standard model.

Gamma-ray burst afterglows are some of the most luminous events known in astronomy and can be observed at virtually any redshift. The furthest spectroscopically confirmed GRB was measured at $z=8.2$ (Tanvir et al. 2009; Salvaterra et al. 2009). Together with their clean synchrotron spectra, they are useful background light beacons for studying the absorption features of interstellar material within their host galaxies, the intergalactic medium, and any intervening material along their lines of sight. Furthermore, being produced by very massive stars, they are normally found in strongly star-forming (SF) galaxies, and within them, not far from their actual birthplaces, since their progenitor stars have rather short lives. This means that GRBs are great tools for stying SF galaxies throughout the Universe. Using GRBs to pinpoint these galaxies gives us the advantage of being able to find even the faintest dwarf SF galaxies (Hjorth et al. 2012; Perley et al. 2016a), which would be missed with regular surveys. As an additional advantage, the galaxies can be studied from within, in absorption, using the light of the afterglow, and later in emission, once the afterglow has faded away.

Since GRBs are the result of the collapse of massive stars, they naturally have an associated supernova (SN; Galama et al. 1998; Hjorth et al. 2003). The SNe associated with GRBs are normally classified as broad-lined Type Ic events, which are relativistic $\mathrm{SNe}$ with fast ejecta responsible for the broadening of the lines. They are also some of the most luminous events, similar in luminosity to Type Ia SNe, and only surpassed by the recently discovered superluminous SNe (Quimby et al. 2011). Their very high kinetic energy is the reason why they have occasionally been called hypernovae (Paczyński 1998). We now know that the characteristics of the SNe associated with GRBs do not significantly correlate with the energy released in gamma rays (Xu et al. 2013; Melandri et al. 2014, but see also Lü et al. 2018). However, we have observed a range of ejecta velocities and supernova luminosities whose origin is not yet fully understood. There are even a few cases of long GRBs where an associated supernovae has not been observed (Fynbo et al. 2006; Della Valle et al. 2006; Michałowski et al. 2018; Tanga et al. 2018), and a long ongoing debate is still trying to determine the reasons.

In this paper we present an extensive data set of observations of GRB 100418A and its host galaxy at a redshift of $z=0.6239$, covering photometric observations from radio to $\mathrm{X}$-rays. This data collection includes unpublished radio observations from GMRT and Ryle; mid-infrared imaging from Spitzer; optical and near-infrared (NIR) imaging from GTC, VLT, Keck, UKIRT, CAHA and WHT, as well as temporally resolved spectroscopic observations from the VLT/X-shooter. GRB 100418A had a bright radio counterpart that was detected for two years after the burst. The optical light curve showed strong deviations from the simple broken power law expected from the fireball model. GRB 100418A is also one of the few cases in which we have good enough data to study the host galaxy in detail, both in absorption and in emission. Finally, the data set that we collected allowed us to search for an associated supernova.

In Sect. 2 we present the observations and data analysis, Sect. 3 we discuss the characteristics of the afterglow evolution, Sect. 4 we explain our search for a supernova component, and Sect. 5 we deal with the host galaxy. In Sect. 6 we present the conclusions of our work. Throughout the paper we adopt a cosmology with a flat universe in which $H_{0}=67.8 \mathrm{~km} \mathrm{~s}^{-1} \mathrm{Mpc}^{-1}$, $\Omega_{m}=0.308$, and $\Omega_{\lambda}=0.692$ (Planck Collaboration XIII 2016). We will follow the convention $F_{v} \propto t^{\alpha} v^{\beta}$ to describe the temporal and spectral evolution of the afterglow. Uncertainties are given at $68 \%(1 \sigma)$ confidence level for one parameter of interest unless stated otherwise, whereas upper limits are given at the $3 \sigma$ confidence level.

\section{Observations}

\subsection{Swift observations}

GRB 100418A was detected by the Burst Alert Telescope (BAT) on-board the Neil Gehrels Swift Observatory (Swift hereafter) on 18 April 2010 at 21:10:08 UT (Marshall et al. 2010, $T_{0}$ hereafter). The gamma-ray emission was characterised by two overlapping peaks, with a duration of $T_{90}=7 \pm 1 \mathrm{~s}$ in the $15-350 \mathrm{keV}$ range (where $T_{90}$ is defined as the time during which $90 \%$ of the photons are collected, from $5 \%$ to $95 \%$ of the total emission), and a hardness ratio (HR) of $\mathrm{HR}=0.89$ (Ukwatta et al. 2010, the HR is defined as the ratio of the fluence in the $50-100 \mathrm{keV}$ band over the fluence in the $25-50 \mathrm{keV}$ band). This identifies GRB 100418A as a long GRB according to the classical division (Kouveliotou et al. 1993), but also as a prototypical intermediate burst, according to the definition of Horváth et al. (2010), with a probability of 0.998 of belonging to this class. The prompt emission was also detected in the soft X-ray band by MAXI SSC (Imatani et al. 2016).

Immediately after the trigger, Swift slewed towards the field to observe with its narrow field instruments. The X-ray Telescope (XRT) began observations $79.1 \mathrm{~s}$ after $T_{0}$ and detected a bright, rapidly fading counterpart. The Ultraviolet/Optical Telescope (UVOT) started observing $87 \mathrm{~s}$ after $T_{0}$ and identified an ultraviolet/optical counterpart at a position consistent with the $\gamma$-ray and X-ray transient. These two instruments performed continued observations of the source for over a month, when it became too faint to be detected by the XRT and its ultraviolet/optical emission was dominated by the host galaxy. In this paper we make use of the X-ray and ultraviolet/optical data publicly available from Swift (Evans et al. 2007, 2009; Marshall et al. 2011).

\subsection{Near-infrared and Optical imaging}

Following the detection of the GRB and its afterglow by Swift, observations were undertaken in the optical and NIR with ground-based telescopes (Pandey et al. 2010; Filgas et al. 2010; Updike et al. 2010; Antonelli et al. 2010; Cucchiara \& Fox 2010; Pearson et al. 2010; Klein et al. 2010; Bikmaev et al. 2010). For this work we gather imaging observations from observatories located across the world in order to obtain a dense coverage of the light-curve evolution.

Our earliest observations began $30 \mathrm{~min}$ after the burst at the $1.5 \mathrm{~m}$ Russian-Turkish Telescope (RTT150), located at TUBITAK National Observatory (Turkey) and were performed using an $R_{C}$ filter. This observatory followed the evolution of the source during the weeks that followed until the light was completely dominated by the host galaxy.

The $10.4 \mathrm{~m}$ Gran Telescopio Canarias (GTC) performed deep optical observations at four epochs between 3 and 90 days in search of the expected supernova component and to study the underlying host galaxy. Further optical images were obtained at different epochs with the European Southern Observatory's (ESO) Very Large Telescope equipped with 


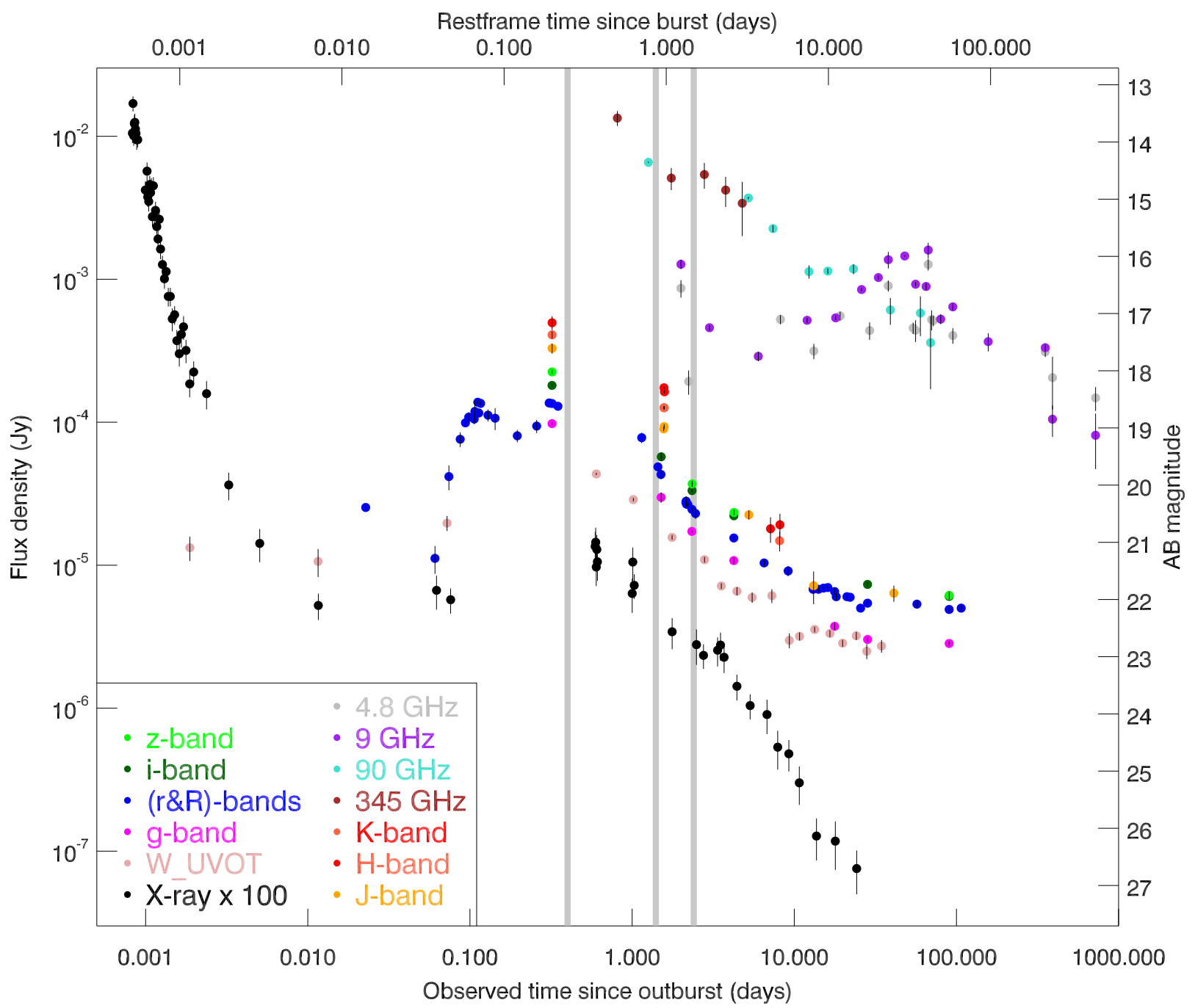

Fig. 1. Light curves of GRB 100418A from X-ray to radio in all bands with significant coverage. Grey vertical lines indicate the times of the $\mathrm{X}$-shooter spectral observations.

FORS2 and X-shooter (as part of the spectroscopic observations), with the $10 \mathrm{~m}$ Keck telescope with LRIS, and with the United Kingdom Infrared Telescope (UKIRT) using the Wide Field Camera (WFCam). The host galaxy was observed when the afterglow and supernovae components where no longer significant, between 3 months and 7 years after the GRB. Our data set includes optical observations from OSIRIS at the $10.4 \mathrm{~m} \mathrm{GTC}$ and LRIS at the $10 \mathrm{~m}$ Keck telescope, NIR data from Omega 2000 at the $3.5 \mathrm{~m}$ CAHA telescope and LIRIS at the $4.2 \mathrm{~m}$ WHT, and mid-infrared data from IRAC onboard the $0.85 \mathrm{~m}$ Spitzer space telescope. The summary of these host observations are shown in Table 5.

Data reduction was performed in a standard fashion using IRAF routines (Tody 1993). Photometric calibration was obtained from field stars present in the SDSS catalogue, 2MASS for the NIR data, and tabulated zero points for Spitzer. All the photometric observations are presented in Table A.1.

Last, we also include observations performed with the $8.2 \mathrm{~m}$ SUBARU telescope using FOCAS, data already published by Niino et al. (2012), as well as early observations of the afterglow by BOOTES-2 (Jelínek et al. 2016).

Throughout the paper we use the equatorial coordinates of the afterglow (J2000) determined from the PdBI millimetre data, with an uncertainty of 0.'15: RA=17:05:27.091, Dec $=+11: 27: 42.29$. Using these coordinates we can estimate the Galactic extinction along the line-of-sight to be $E(B-V)=$ 0.065 mag (Schlafly \& Finkbeiner 2011).

\subsection{Submillimetre, millimetre and radio observations}

Submillimetre (submm) data were obtained from the Submillimeter Array (SMA), in Hawaii (USA), starting $16 \mathrm{~h}$ after the burst. For this first observation we used 7 out of the 8 SMA antennas, under good weather conditions, with zenith opacities at $225 \mathrm{GHz}$ of $\tau \sim 0.06$ (precipitable water vapour, PWV $\sim 1 \mathrm{~mm}$ ). Titan and Neptune were used as flux calibrators and 3C454.3 as bandpass calibrator. Atmospheric gain was corrected by observing the nearby quasar J1751+096 every $15 \mathrm{~min}$. With these data we discovered a very bright counterpart (Martin et al. 2010) at a flux of $13.40 \pm 1.60 \mathrm{mJy}$. Observations continued over the following four nights, tracking the evolution of the afterglow until it became undetectable on 23 April.

Following the submm detection we observed the burst from Plateau de Bure Observatory (PdBI) of the Institut de Radioastronomie Millimétrique (IRAM). The observations were performed in the $3 \mathrm{~mm}$ band and cover the temporal range 
Table 1. Radio observations.

\begin{tabular}{cccc}
\hline \hline $\begin{array}{c}T-T_{0} \\
(\text { days })\end{array}$ & Observatory & $\begin{array}{c}\text { Frequency } \\
(\mathrm{GHz})\end{array}$ & $\begin{array}{c}\text { Flux } \\
(\mathrm{mJy})\end{array}$ \\
\hline 2.2315 & WSRT & 4.8 & $0.193 \pm 0.037$ \\
8.2150 & WSRT & 4.8 & $0.524 \pm 0.039$ \\
13.2010 & WSRT & 4.8 & $0.314 \pm 0.038$ \\
19.1850 & WSRT & 4.8 & $0.554 \pm 0.041$ \\
29.1575 & WSRT & 4.8 & $0.438 \pm 0.061$ \\
54.0895 & WSRT & 4.8 & $0.456 \pm 0.041$ \\
\hline 4.7669 & Ryle & 15.0 & $0.90 \pm 0.12^{a}$ \\
7.8410 & Ryle & 15.0 & $2.70 \pm 0.12^{a}$ \\
8.8539 & Ryle & 15.0 & $1.80 \pm 0.11^{a}$ \\
9.7480 & Ryle & 15.0 & $1.70 \pm 0.14^{a}$ \\
13.8820 & Ryle & 15.0 & $1.10 \pm 0.18^{a}$ \\
23.7130 & Ryle & 15.0 & $0.70 \pm 0.11^{a}$ \\
43.7400 & Ryle & 15.0 & $1.50 \pm 0.12^{a}$ \\
\hline 1.2603 & PdBI & 103.1 & $6.57 \pm 0.07$ \\
5.2114 & PdBI & 86.7 & $3.70 \pm 0.07$ \\
7.3930 & PdBI & 86.7 & $2.26 \pm 0.13$ \\
12.3284 & PdBI & 105.7 & $1.13 \pm 0.12$ \\
16.1079 & PdBI & 86.7 & $1.14 \pm 0.05$ \\
23.1766 & PdBI & 86.7 & $1.18 \pm 0.09$ \\
39.1444 & PdBI & 102.6 & $0.61 \pm 0.13$ \\
59.9582 & PdBI & 86.7 & $0.58 \pm 0.18$ \\
69.2371 & PdBI & 99.5 & $0.36 \pm 0.19$ \\
\hline 0.8110 & SMA & 350 & $13.4 \pm 1.6$ \\
1.7448 & SMA & 350 & $5.1 \pm 0.9$ \\
2.7891 & SMA & 350 & $5.4 \pm 1.1$ \\
3.7744 & SMA & 350 & $4.2 \pm 1.0$ \\
4.7754 & SMA & 350 & $3.4 \pm 1.4$ \\
\hline & & & \\
\hline
\end{tabular}

Notes. ${ }^{(a)}$ Blended with the nearby source NVSS J170527.66 +112706.9 .

between 1.3 and 70 days after the GRB. Both the SMA and the PdBI data were already published in the compilation of de Ugarte Postigo et al. (2012a).

Observations at $15 \mathrm{GHz}$ were performed by the Ryle telescope. However, these observations are affected by blending with a bright nearby source, NVSS J170527.66 +112706.9. The afterglow is well detected between day 7 and day 10, but the rest of the epochs are probably dominated by the contribution of the contaminating source.

The afterglow of GRB 100418A was detected with the Westerbork Synthesis Radio Telescope (WSRT) at $4.8 \mathrm{GHz}$ two days after the burst at a flux density of about $200 \mu \mathrm{Jy}$. WSRT subsequently followed up the evolution of this afterglow at $4.8 \mathrm{GHz}$ with a bandwidth of $8 \times 20 \mathrm{MHz}$ and the bright radio source $3 \mathrm{C} 286$ as a primary flux calibrator. The observations have been analysed using the Astronomical Image Processing Software (AIPS) ${ }^{1}$ and the Multichannel Image Reconstruction Image Analysis and Display (MIRIAD, Sault et al. 1995) software package.

The details of our observations and the results are shown in Table 1 . In addition to this, we use the available literature data obtained from the Very Large Array (VLA), Australia Telescope compact Array (ATCA) and Very Long Baseline Array (VLBA) at 4-9 GHz for our analysis (Moin et al. 2013).

1 http://www.aips.nrao.edu/
Table 2. Log of spectroscopic observations obtained with X-shooter at VLT.

\begin{tabular}{cccc}
\hline \hline $\begin{array}{c}T-T_{0} \\
\text { (days) }\end{array}$ & Arm & $\begin{array}{c}\text { Exposure } \\
(\mathrm{s})\end{array}$ & $\begin{array}{c}\text { Average } \\
S / N\end{array}$ \\
\hline \multirow{3}{*}{0.375} & UVB & $4 \times 1200$ & 35 \\
& VIS & $4 \times 1200$ & 43 \\
& NIR & $8 \times 600$ & 17 \\
\hline \multirow{3}{*}{1.470} & UVB & $4 \times 1200$ & 19 \\
& VIS & $4 \times 1200$ & 22 \\
& NIR & $8 \times 600$ & 6.9 \\
\hline \multirow{3}{*}{2.487} & UVB & $4 \times 1200$ & 10 \\
& VIS & $4 \times 1200$ & 13 \\
& NIR & $8 \times 600$ & 3.1 \\
\hline
\end{tabular}

\subsection{Spectroscopy}

As soon as the field became observable from Paranal Observatory (Chile), we obtained follow-up spectroscopic observations of the afterglow of GRB 100418A using X-shooter (Antonelli et al. 2010). X-shooter (Vernet et al. 2011) is a triple spectrograph capable of observing simultaneously a complete spectrum between 3000 and $24800 \AA$ at intermediate resolution. In our case, we used slit widths of of $1^{\prime \prime}, 0 .{ }^{\prime \prime} 9$ and $0 .{ }^{\prime \prime} 9$ in the UVB, VIS and NIR arms, respectively, with a $2 \times 1$ binning (binning in the spectral but not in the spatial direction), which delivers a resolution of 5100, 8800 and 5300 in each arm.

Thanks to the peculiar evolution of the afterglow, we were able to obtain a high signal-to-noise $(\mathrm{S} / \mathrm{N})$ ratio starting $8.37 \mathrm{~h}$ after the burst onset, when the afterglow was near its maximum brightness, at $R_{C}=18.1 \pm 0.1$ (Antonelli et al. 2010). This spectrum presents abundant absorption and emission features over the entire spectral range of X-shooter at a redshift of $z=0.6239 \pm 0.0002$, the redshift of the GRB.

The sustained brightness of the afterglow allowed further observations with $\mathrm{X}$-shooter over the following nights. In total, three epochs were obtained at 0.4, 1.5 and 2.5 days after the burst, with $\mathrm{S} / \mathrm{N}$ of 43,22 and 13 per spectral bin in the VIS arm, respectively (de Ugarte Postigo et al. 2011). Each one of the three observations consisted of 4 different exposures of $1200 \mathrm{~s}$ each; for the NIR arm each exposure was split in two individual ones of $600 \mathrm{~s}$ each. The exposures were taken using the nodding along the slit technique with an offset of $5^{\prime \prime}$ between exposures in a standard ABBA sequence. The log of spectroscopic observations is displayed in Table 2.

We processed the spectra using the $\mathrm{X}$-shooter data reduction pipeline (Goldoni et al. 2006; Modigliani et al. 2010) and further scripts, as described by Selsing et al. (2018). To perform flux calibration we extracted a spectrum from a staring observation of the flux standard GD153 (Bohlin et al. 1995) taken the same night for each observation. Each of the spectra were matched to the multiband photometry interpolated to the mean time of each spectrum. For each epoch we performed two different extractions. First we use optimal extraction, which is best for the study of the afterglow. Then we used one with a fixed aperture that allowed us to cover the full extension of the host galaxy. This second extraction provides us with better measurements for the study of the host. The spectra are publicly available through the GRBSpec database ${ }^{2}$ (de Ugarte Postigo et al. 2014).

\footnotetext{
2 http://grbspec.iaa.es
} 


\section{Afterglow analysis}

\subsection{Light curves}

The first five minutes of the X-ray light curve showed a very sharp decline (with a temporal index of $\alpha=-4.23_{-0.16}^{+0.17}$ Pagani et al. 2010), probably related to the tail of the prompt burst emission. This was followed by a flattening and subsequent increase in flux density, reaching a maximum around 0.5 days after the burst. After this, the light curve decayed with a steepening slope until it faded beyond the sensitivity limit of XRT 25 days after the burst.

The optical light curve was characterised by an early shallow decay, reaching a minimum at around $1.5 \mathrm{~h}$ after the burst, after which there was a very steep increase in brightness of a factor of ten within one hour. After this step in the light curve the brightness was sustained, with perhaps a shallow decay until around one day, when the light curve steepened and decayed for the following 2 weeks, when it became dominated by the contribution of the host galaxy.

The submm and mm light curves at $350 \mathrm{GHz}$ and $90 \mathrm{GHz}$ evolved both in a similar way. There was a fast decay between the first observation obtained 0.8 days after the burst and day 1.5, after which the light curve first flattened for a day and then resumed its decay until day seven, when a second plateau stabilised the flux density until day 23 . The light curve then decays and becomes too faint to be detected two months after the burst. At the time of discovery, this was the second brightest $\mathrm{mm} / \mathrm{submm}$ counterpart detected (after GRB 030329 , which peaked at $\sim 70 \mathrm{mJy}$; Sheth et al. 2003; Resmi et al. 2005), and is currently still the 4th (after GRB 171205A, de Ugarte Postigo et al. 2017 and GRB 100621A, Greiner et al. 2013), see Fig. 2.

At $4.8 \mathrm{GHz}$, the light curve rose during the two months of observations that were performed with the WSRT. However, oscillations in the flux are apparent during the first 3 weeks, probably due to scintillation, as is commonly seen at these frequencies (Goodman 1997). Observations from the literature (Moin et al. 2013) also showed a peak in radio that was reached at around two months, followed by a steady decay.

Figure 2 shows the brightness of GRB 100418A as compared to two samples of $\mathrm{mm} / \mathrm{submm}$ (de Ugarte Postigo et al. 2012a) and radio afterglows (Chandra \& Frail 2012). Although it is among the brightest events ever observed, the actual luminosity is not extraordinary, with a group of GRBs reaching luminosities almost an order of magnitude more than this event.

\subsection{Intrinsic dust extinction along the GRB line of sight}

We performed fits of the spectral energy distribution from NIR to X-rays at 0.4 days once the photometric data had been corrected for the foreground extinction stemming from our own Galaxy (Schlafly \& Finkbeiner 2011). We tested three intrinsic dust models (Small Magellanic Cloud - SMC, Large Magellanic Cloud - LMC, and Milky Way - MW), and underlying simple and broken power-law spectra. The statistically preferred fit was obtained with an SMC extinction law and single power law, although at this redshift all the other dust laws are not ruled out and give comparable results (Kann et al. 2006; Starling et al. 2007; Zafar et al. 2011; Schady et al. 2012). If a broken power law is forced, the break is found at energies larger than $\mathrm{X}$ rays, confirming the existence of a single power-law spectrum between optical and X-rays. We performed $\chi^{2}$ statistics for optical data and C-stat statistics for X-ray data. All errors are at $90 \%$

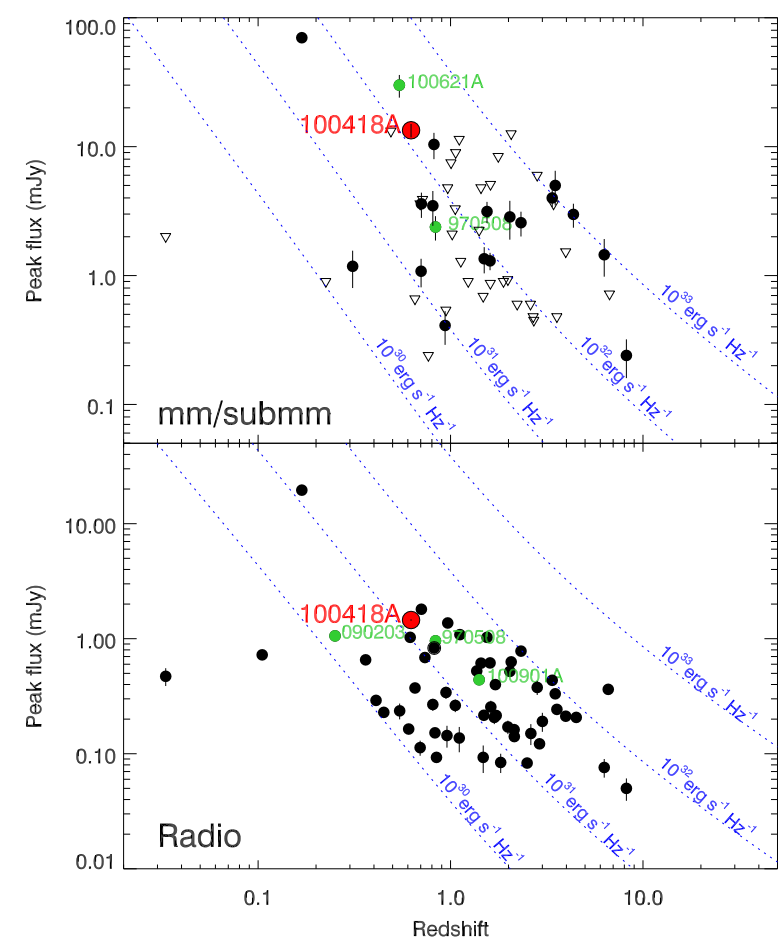

Fig. 2. Peak flux densities of GRB 100418 A as compared to the $\mathrm{mm} / \mathrm{submm}$ and radio samples from de Ugarte Postigo et al. (2012a) and Chandra \& Frail (2012). In blue we indicate lines of equal luminosity, with the most luminous events being found in the top right region of the plots. We have highlighted in green other GRBs where strong rebrightenings have been observed (see Sect. 3.3).

confidence. Our best fit implies an SMC extinction law with an $A_{V}=0.086 \pm 0.039 \mathrm{mag}$.

Several other works have already performed some kind of fit to estimate the line-of-sight extinction towards GRB 100418A. The values that we derived are consistent with the measurement recently performed by Zafar et al. (2018), who determined that the NIR to X-rays SED could be well explained by using a single power law and a featureless extinction curve with $R_{V}=$ $2.42_{-0.10}^{+0.08}$ and $A_{V}=0.12 \pm 0.03 \mathrm{mag}$. This is also in accordance with the findings of Marshall et al. (2011) who describe the data with a single power law, and an SMC extinction of $A_{V}=$ $0.056 \pm 0.048 \mathrm{mag}$. We also note that our fits are inconsistent with those performed by Japelj et al. (2015), who found their best solution with a broken power law and an $A_{V}=0.20_{-0.02}^{+0.03} \mathrm{mag}$. Each of these works used slightly different methods and calibrations.

\subsection{Broadband afterglow evolution within the fireball model}

GRB 100418A is one of the members of a growing class of GRBs that exhibit a fast rebrightening in their optical light curves within the first day after the gamma-ray trigger. Previous studies of this particular GRB have suggested different explanations for this feature, such as continuous injection of energy into the blast wave moving through an ambient medium, or an offaxis jet viewing angle (Marshall et al. 2011; Laskar et al. 2015). The injection of energy could be due to ejecta with a wide range of Lorentz factors or continued activity of the central engine, and previous multiwavelength studies have indicated that in the case of GRB 100418A the continued activity of the central engine is favoured because the derived physical parameters for the other energy injection model are not plausible (Marshall et al. 2011; 
Table 3. Determination of intrinsic extinction laws through SED fitting.

\begin{tabular}{ccccc}
\hline \hline $\begin{array}{c}\text { Ext. } \\
\text { law }\end{array}$ & $\begin{array}{c}A_{V} \\
(\mathrm{mag})\end{array}$ & $\begin{array}{c}N_{\mathrm{H}} \\
10^{22} \mathrm{~cm}^{-2}\end{array}$ & $\beta$ & $\chi^{2} /$ d.o.f. \\
\hline SMC & $0.086 \pm 0.039$ & $0.242_{-0.133}^{+0.170}$ & $-1.061_{-0.023}^{+0.024}$ & $90.25 / 95$ \\
LMC & $0.091 \pm 0.041$ & $0.243_{-0.134}^{+0.171}$ & $-1.062_{-0.023}^{+0.024}$ & $90.32 / 95$ \\
MW & $0.092 \pm 0.042$ & $0.242_{-0.133}^{+0.171}$ & $-1.062_{-0.023}^{+0.024}$ & $90.50 / 95$ \\
\hline
\end{tabular}

Notes. Fits shown here were performed with a single power-law spectrum.

Moin et al. 2013; Laskar et al. 2015). Previous studies, and in particular Laskar et al. (2015), performed a detailed broadband modelling of the evolution of this event, which we will not attempt to repeat here. However, they did not have or did not model the detailed optical to NIR range light curves between $\sim 0.1$ and $\sim 1$ day after the gamma-ray trigger. The light curve shows that after the very steep rise, there is a plateau that lasts a large fraction of the first day. Here we will focus on the steep rise, plateau, and subsequent "normal" afterglow decay, and possible explanations for the observed behaviour.

First of all, we determine the spectral energy distributions (SEDs) from NIR to X-ray frequencies for several epochs with good spectral coverage and for which the host galaxy contribution had been removed. This analysis was done at $0.60,1.77$ and 2.56 days, in a similar fashion to what was done in the previous section. We loaded the XRT spectra and NIR/optical magnitudes into XSPEC, and fit the SEDs with single and broken power-law functions. To account for optical extinction we used MW, SMC and LMC extinction models, and determined that there were no large differences with the different extinction laws due to the small amount of extinction and the low redshift. The main result is that the spectrum can be fit well with a single power law from NIR to X-ray frequencies at all epochs, and that a spectral break does not improve the fit. The spectral index is $\beta=-1.02 \pm 0.03$, $\beta=-1.05 \pm 0.06$ and $\beta=-1.00 \pm 0.05$ for each of the three epochs, respectively, indicating that there is no significant spectral evolution; most notably, that there is no spectral evolution from the plateau phase to the "normal" decay phase. Unfortunately there is insufficient spectral coverage during the lightcurve rise, and it is thus not possible to check for any spectral evolution at early times. For further considerations we adopt the spectral index of the 0.6 day epoch, i.e. $\beta=-1.02 \pm 0.03$, as the spectral index for all three light-curve phases.

To compare our light curve with a standard fireball model, we look at the evolution after day 1 , when the behaviour corresponds to that of a typical afterglow. Since the optical emission is affected by a strong contribution from the host galaxy and a possible supernova (see Sects. 4 and 5), we use the X-rays to perform a light curve evolution fit, as it is expected to contain only afterglow contribution. We find that beyond 1 day the Xray light curve is well described by a broken power-law fit with a break time at $t_{\mathrm{b}}=4.2 \pm 1.1$ day and temporal slope indices of $\alpha_{1}=-0.98 \pm 0.10$ and $\alpha_{2}=-1.91 \pm 0.16$. This break in the light curve is consistent with the jet break that we would expect to find in a collimated afterglow emission. Given that the spectral fits show that optical and X-ray data were within the same spectral segment, the X-ray afterglow light curve and the optical afterglow are expected to evolve in the same way. Indeed, in Sect. 4 we show that the optical and X-ray afterglow evolution are consistent after day 2 .

To further constrain the physics of the blast wave, we use the typical relations for the forward-shock model between the

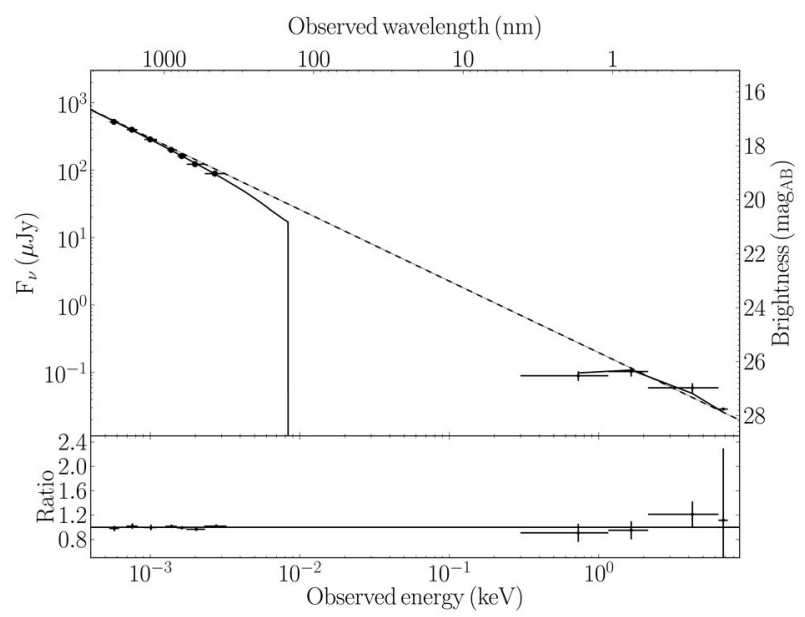

Fig. 3. Fit of the SED at 0.4 days from NIR to X-rays with a single power law and an SMC extinction law.

spectral index, the temporal indices, and the power-law index $p$ of the electron energy distribution (also known as the "closure relations", Gao et al. 2013). We find that the evolution is consistent with that of an afterglow expanding in the interstellar medium, where the cooling break would lie redwards of the opti$\mathrm{cal} / \mathrm{NIR}$ data. This is consistent with what has been previously proposed for this event (Marshall et al. 2011; Moin et al. 2013; Laskar et al. 2015). In such a scenario we can use the different spectral and temporal indices to derive the value of $p$ : From the post-break slope we obtain that $p=-\alpha_{2}=1.91 \pm 0.16$, from the pre-break decay $p=\left(2-4 \alpha_{1}\right) / 3=1.97 \pm 0.14$, and from the spectral slope $p=-2 \beta=2.04 \pm 0.06$. Combining them all, we can derive a best estimate of $p=2.02 \pm 0.05$. This value is not far from the value of $p=2.14$ estimated by Laskar et al. (2015) from broadband modelling and within typical values for GRB afterglows (e.g. Starling et al. 2008; Curran et al. 2010; Kann et al. 2018).

Given the value for $p$, we derive the rate of energy injection into the forward shock during the plateau phase. We adopt the convention from van Eerten (2014) that the injected luminosity is proportional to $t_{\mathrm{in}}^{q}$, with $t_{\text {in }}$ the energy injection timescale, i.e. the injected energy is proportional to $t_{\text {in }}^{1+q}$. We fit the opticalto-NIR light curves during the plateau (for which we assume a temporal slope of $0.07 \pm 0.07$ ), from 0.1 to 0.8 days after the burst, and find $q=-0.08 \pm 0.09$, i.e. energy injection at a constant rate. We note that this energy injection rate was not derived by earlier studies, because they did not have or did not model this part of the light curves.

The steep rise between 0.05 and 0.1 days is much harder to interpret within the standard forward-shock model. The temporal slope is $\alpha_{\text {rise }}=5.4 \pm 1.1$ (using as origin the GRB trigger time, $T_{0}$ ), and if one would interpret this as an energy injection, this would lead to $q=5.3 \pm 1$. , which seems to be quite extreme but not unfeasible. The energy increase in this time interval is a factor of $24_{-9}^{+15}$, while it is a factor of $6.6 \pm 0.4$ during the plateau phase. If one chooses to interpret the energy injection as ejecta with a wide range of Lorentz factors, the shallow decay phase can be interpreted in this way. In this theoretical framework, it is assumed that the amount of mass in the ejecta with a Lorentz factor greater than $\gamma$ is a power-law function of $\gamma$ with powerlaw index $s$ (e.g. Zhang et al. 2006). The value we derived for $q$ results in $s=4.5 \pm 0.5$ for a fireball expanding in a constant density medium, which is reasonable, but the value for $s$ is very poorly constrained for a wind density medium $\left(\rho \propto r^{-2}\right)$. Seeing 


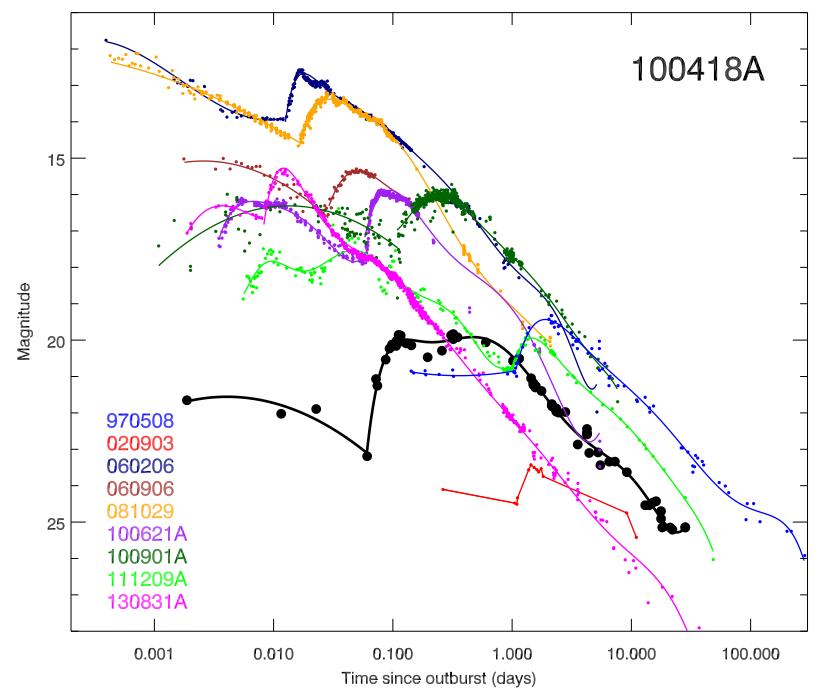

Fig. 4. Comparison of the optical light curve of GRB 100418A (in black) with other GRB afterglows showing steep rebrightenings. All the light curves have been shifted to a redshift of $z=1$ to allow for a direct comparison. When enough data are available the light curves have been fitted with two spline curves, one before the rebrightening and a second one after. When possible the contribution of the host galaxy has been subtracted.

these numbers, one would have a preference for the constant density scenario, as the $\mathrm{s}$ value is more reasonable. However, in the steep rise phase, the value for $s$ is unphysical (in both environment density scenarios), and therefore the latter phase cannot be interpreted in this way. Taking all of this into account, we agree with the general conclusion of previous studies that a model of continued activity of the central engine is favoured over ejecta with a wide range of Lorentz factors (Marshall et al. 2011; Moin et al. 2013; Laskar et al. 2015), but our detailed analysis of the light curves and spectra leads to different conclusions on the rate of energy injection and details of the physics of the forward shock.

In Fig. 4 we compare the light curve of GRB 100418A with those of other GRB light curves with a similar steep rebrightening (from Kann et al. 2006, 2010, 2016, 2018, and in prep.). In Table 4 we compare some light curve parameters of GRB 100418A with those of these other GRBs.

These steps have been detected for a number of GRB afterglows. They tend to happen during the first day and have an optical luminosity increase ranging between 1 and 3 magnitudes. They are characterised by a very steep rise slope with typical values of $3<\alpha<5$ but that can reach values of $\alpha \sim 15$. When enough multiband data has been available, a colour evolution has been evident (e.g. GRB 081029, Nardini et al. 2011; GRB 100621A, Greiner et al. 2013; GRB 111209A, Kann et al. 2018). The afterglow is normally bluer before the step. The temporal slope is steeper in the redder bands, and the amplitude of the step is also larger in the redder bands. This is consistent with the onset of a different spectral component, however the actual nature of these bumps is not yet clear.

\section{The faint supernova associated to GRB 100418 A}

The search for a supernova component in the light curve of GRB 100418A is strongly affected by the light of the host galaxy, which is more than a magnitude brighter than what we would expect for a contribution similar to SN $1998 \mathrm{bw}$ at this redshift.
Table 4. Comparison of the characteristics of light curve rebrightenings seen for other GRB afterglows.

\begin{tabular}{cccc}
\hline \hline GRB & $\alpha_{\text {rise }}$ & $\Delta$ mag & $t_{\text {rise }}$ (day) \\
\hline $100418 \mathrm{~A}$ & $5.4 \pm 1.1$ & $3.4 \pm 0.5$ & $0.062 \pm 0.005$ \\
\hline 970508 & $3.93 \pm 0.12$ & $1.41 \pm 0.12$ & $1.08 \pm 0.03$ \\
020903 & $2.97 \pm 0.10$ & $1.02 \pm 0.10$ & $1.10 \pm 0.02$ \\
060206 & $5.43 \pm 0.20$ & $1.27 \pm 0.12$ & $0.0125 \pm 0.0010$ \\
060906 & $2.02 \pm 0.28$ & $1.28 \pm 0.40$ & $0.029 \pm 0.002$ \\
081029 & $4.33 \pm 0.21$ & $1.19 \pm 0.14$ & $0.0165 \pm 0.0010$ \\
$100621 \mathrm{~A}$ & $15.8 \pm 1.32$ & $1.96 \pm 0.16$ & $0.058 \pm 0.002$ \\
$100901 \mathrm{~A}$ & $4.09 \pm 0.55$ & $1.45 \pm 0.25$ & $0.110 \pm 0.005$ \\
$111209 \mathrm{~A}$ & $3.74 \pm 0.18$ & $0.90 \pm 0.05$ & $1.007 \pm 0.010$ \\
$130831 \mathrm{~A}$ & $2.83 \pm 0.11$ & $1.41 \pm 0.14$ & $0.0082 \pm 0.0003$ \\
\hline
\end{tabular}

Notes. The values have been calculated after shifting all the light curves to $z=1$.

To get reliable photometry at the time when one could expect a $\mathrm{SN}$ to emerge, image subtraction using a host galaxy template is mandatory.

We used our deep GTC/OSIRIS images to obtain imagesubtracted magnitudes of the optical emission associated with GRB 100418A, using the final epoch in each filter as a template. Image subtraction was performed using an adaptation of the original ISIS programme (Alard \& Lupton 1998; Alard 2000) that was developed for the Hubble Space Telescope SN surveys by Strolger et al. (2004), and used in previous GRBSN studies (Cano et al. 2011, 2014, 2017a). A key advantage of this code is the option to specify a set of stamps that the programme uses when it calculates the point-spread function in each image. The image-subtraction technique was then optimised by varying the kernel mesh size and measuring the standard deviation $(\sigma)$ of the background counts in a nearby region in the image (where images with lower $\sigma$ values indicate that they are a better subtracted image). As a self-consistency check, we compared the magnitudes of the optical transient (OT, i.e. the combination of afterglow and $\mathrm{SN}$ emission) against those found by performing photometry on the un-subtracted images, converting the magnitudes into fluxes, and then mathematically subtracting the host flux. In images where the OT was detected in the subtracted image, excellent agreement was obtained with both methods, showing that the image-subtraction technique was well-optimised. Using this method, an OT was seen at the position of the GRB in the subtracted images, $28.3 \mathrm{~d}$ after the burst, or $17.4 \mathrm{~d}$ in rest frame, when the supernovae would be expected to dominate the emission with photometric measurements corresponding to $g^{\prime}=25.64 \pm 0.27 \mathrm{mag}, r^{\prime}=24.81 \pm 0.12 \mathrm{mag}$ and $i^{\prime}=23.79 \pm 0.08 \mathrm{mag}$.

The magnitudes calculated above are a contribution of both afterglow and supernova. To estimate the actual luminosity of the $\mathrm{SN}$, we need to determine the fraction of the light that comes from the afterglow at this time. To do this, we use the afterglow fit described in the previous section. In Fig. 5 we have used the fit of the X-ray data (in black) and applied it to the optical light curves, which are well reproduced by this fit beyond day 2 . At late times we see that the $g^{\prime}$-band emission measured through image subtraction (big dots) is consistent with being due to only afterglow, but that both $r^{\prime}$ and $i^{\prime}$ bands show a clear excess due to a supernova component. Comparing to the expected flux for a supernova with the same luminosity as SN 1998bw (corrected for a redshift of $z=0.6239$, dotted lines), we can already see that the supernova that we measure is significantly fainter than 


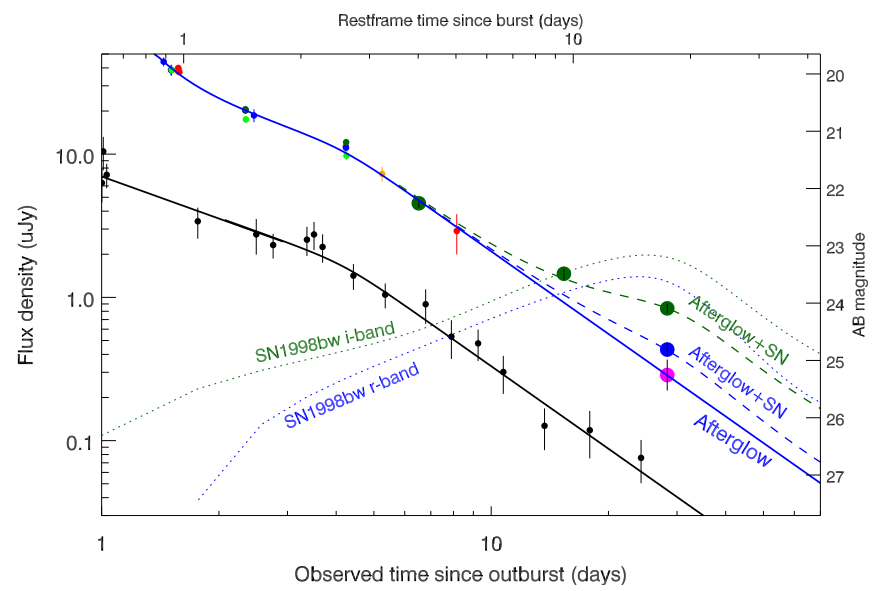

Fig. 5. Late light curve of GRB 100418A in X-rays and optical, where the contribution of the host galaxy has been removed. All the optical bands have been offset to the $r^{\prime}$-band for clarity. Only data with $\mathrm{S} / \mathrm{N}$ above 3, after host subtraction are plotted. At late times image subtraction techniques (thick dots) show that there is an excess of emission in the $r^{\prime}$ and $i^{\prime}$ bands, corresponding to a faint supernova. Solid lines correspond to the afterglow contribution in X-rays and optical. The dashed lines show the combined contribution of afterglow and supernova (for which we used SN 1998bw templates) scaled to match the late photometry. The dotted lines show the expected light curves for SN 1998bw at the redshift of GRB 100418A. Colour coding is equivalent to that of Fig. 1.

the usual standard for GRBs. We perform a similar analysis with the VLT/FORS data in the $I_{C}$ band on days 6 and 15, compared with the same filter on Keck/LIRIS. Although the subtraction is not as clean in this case due to the difference in instruments, the photometry of the residuals is consistent with the GTC results, indicating an excess in redder optical bands with respect to the afterglow.

We compare the luminosity of the SN of GRB 100418A with SN 1998bw by applying a multiplying factor to the supernova template and combining it with the afterglow emission to match the subtracted photometry. In this way, we find that in $i^{\prime}$-band the $\mathrm{SN}$ is $29 \%$ and in $r^{\prime}$-band $11 \%$ of the luminosity of SN 1998bw. By subtracting the known contribution of the afterglow to our photometry, we can estimate the magnitude of the supernova associated to GRB 100418A, at the time of the GTC observation, to be $g^{\prime}>25.82 \mathrm{mag}, r^{\prime}=$ $25.99 \pm 0.36 \mathrm{mag}$ and $i^{\prime}=24.52 \pm 0.12 \mathrm{mag}$. Although the epoch of the GTC observation is slightly after the maximum expected for a SN 1998bw-like event, the difference between the peak and the observed epoch (for the SN1998bw template) would have been of $0.09 \mathrm{mag}$ in the $r^{\prime}$-band and 0.04 in the $i^{\prime}$ band, meaning that these observations should correspond, within errors, to the peak magnitude of the supernova. They are, in any case, the best approximation that we can have for this event.

At a redshift of $z=0.6239$, the distance modulus of GRB 100418A corresponds to $\mu=42.91 \mathrm{mag}$. With this, we can calculate the absolute magnitudes corresponding to the observed bands to be, in rest frame wavelengths $M_{2900 \AA}>-17.09 \mathrm{mag}$, $M_{3800 \AA}=-16.92 \pm 0.36 \mathrm{mag}, M_{4600 \AA}=-18.40 \pm 0.12 \mathrm{mag}$. The observer frame $r^{\prime}$ and $i^{\prime}$ bands are roughly consistent with rest frame $u^{\prime}$ and $g^{\prime}$ bands, respectively.

The limited cadence of the optical light curves at this time limits how much we can say about the observational properties of the associated supernova, i.e. we cannot find the relative stretch and luminosity factors relative to the archetype

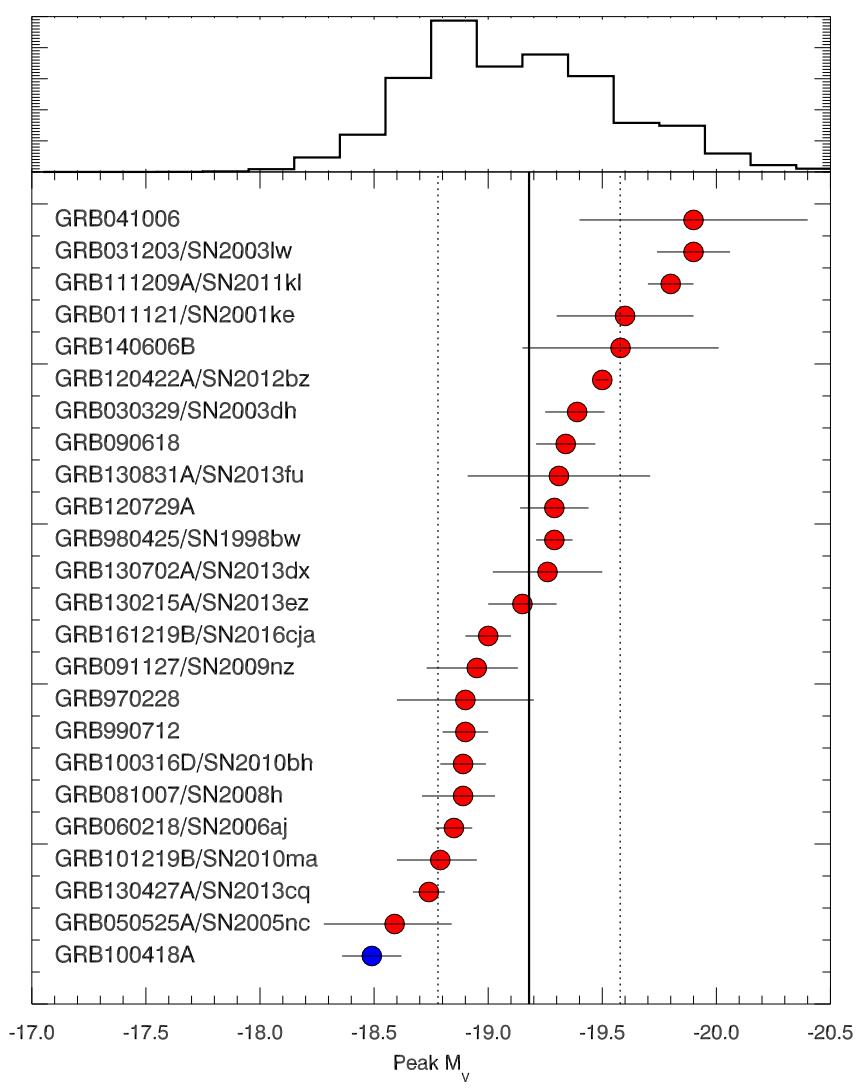

Fig. 6. Peak absolute magnitudes in the rest frame $V$-band of a sample of GRB-SNe. The vertical solid line indicates the average of the sample, whereas the dotted lines indicate the standard deviation from this value. Top panel: histogram of the $M_{V}$ distribution. GRB 100418A, shown in blue, is the faintest supernova within this sample.

GRB-SN 1998bw (e.g. Zeh et al. 2004; Cano 2014). However, we can compare the absolute magnitude of the GRB 100418A $\mathrm{SN}$ with the literature by assuming that the absolute magnitude derived from the $i^{\prime}$-band observation can be assimilated to a rest frame $V$-band, that once corrected for the line-of-sight extinction results in $M_{V}=-18.49 \pm 0.13 \mathrm{mag}$. For example, Richardson (2009) found, for a sample of 14 GRB-SNe, an average peak $V$-band magnitude of $\bar{M}_{V}=-19.0 \mathrm{mag}$ with a standard deviation of $0.8 \mathrm{mag}$. Moreover, referring to the sample of GRB-SNe by Li \& Hjorth (2014), the faintest GRB-SNe to date were SN 2010bh $\left(M_{V}=-18.89 \mathrm{mag}\right)$ and SN 2006aj $\left(M_{V}=\right.$ $-18.85 \mathrm{mag}$ ). As such, the SN associated with GRB 100418A is amongst the faintest, if not the faintest, GRB-SN yet detected.

Using data from Richardson (2009), Li \& Hjorth (2014), Cano et al. (2014, 2015, 2017a,b), Olivares (2015), Becerra et al. (2017), Kann et al. (2018; and references therein) we have either taken the peak $M_{V}$ calculated in the papers for the corresponding $\mathrm{SNe}$, or derived our own estimate, approximating from the closest band to the rest frame $V$-band. Figure 6 shows a comparison of the magnitudes of this sample. We obtain that the average value for the sample is $\bar{M}_{V}=-19.2 \mathrm{mag}$, with a standard deviation of $0.4 \mathrm{mag}$. The average value is slightly brighter than the one derived for the sample of Richardson (2009), and with a tighter standard deviation. We note that we did not use the $3 \mathrm{SNe}$ that were already marked in the sample of Richardson (2009) as highly uncertain. Within this sample, the SN associated with GRB 100418A would be the faintest detected to date. 


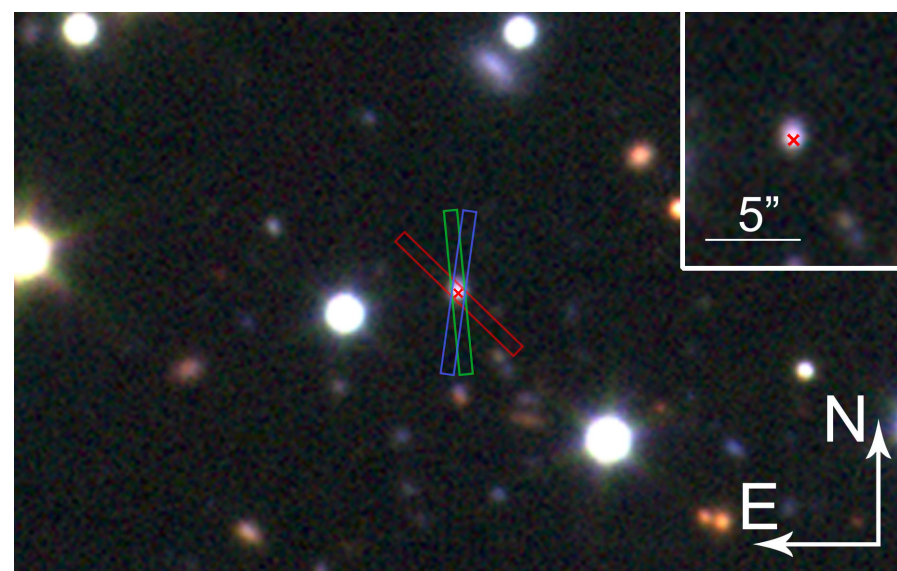

Fig. 7. Image of the host galaxy field and the three slit positions of the three different $\mathrm{X}$-shooter epochs (epoch 1 is red, epoch 2 green and epoch 3 is blue). The GRB position is indicated with a cross.

Table 5. Host galaxy magnitudes, in the $\mathrm{AB}$ system, corrected for Galactic extinction, together with the magnitudes derived from the model fit.

\begin{tabular}{cccc}
\hline \hline Filter & Telescope/Instrument & $\mathrm{AB}_{\text {obs }}$ mag & $\mathrm{AB}_{\text {model }}$ mag \\
\hline$u$ & $10.4 \mathrm{~m}$ GTC/OSIRIS & $22.86 \pm 0.06$ & 22.829 \\
$B$ & $10 \mathrm{~m}$ Keck/LRIS & $22.82 \pm 0.10$ & 22.817 \\
$g$ & $10.4 \mathrm{~m}$ GTC/OSIRIS & $22.75 \pm 0.09$ & 22.775 \\
$r$ & $10.4 \mathrm{~m}$ GTC/OSIRIS & $22.26 \pm 0.06$ & 22.288 \\
$i$ & $10.4 \mathrm{~m}$ GTC/OSIRIS & $21.95 \pm 0.06$ & 21.924 \\
$I$ & $10 \mathrm{~m}$ Keck/LRIS & $21.87 \pm 0.06$ & 21.898 \\
$z$ & $10.4 \mathrm{~m}$ GTC/OSIRIS & $21.94 \pm 0.07$ & 21.939 \\
$J$ & $3.5 \mathrm{~m}$ CAHA/O2000 & $21.89 \pm 0.14$ & 21.861 \\
$H$ & $3.5 \mathrm{~m}$ CAHA/O2000 & $21.92 \pm 0.30$ & 21.733 \\
$K_{S}$ & $4.2 \mathrm{~m}$ WHT/LIRIS & $21.55 \pm 0.21$ & 21.766 \\
$3.6 \mu \mathrm{m}$ & $0.85 \mathrm{~m}$ Spitzer/IRAC & $21.98 \pm 0.10$ & 21.981 \\
\hline
\end{tabular}

\section{Host galaxy analysis}

The host of GRB 100418A is a SF dwarf galaxy with a slight elongation in the north-south direction (see Fig. 7). The apparent physical size of the galaxy is $(7.3 \pm 0.6) \times(6.0 \pm 0.5) \mathrm{kpc}$ $\left(1\right.$.' $^{\prime} 5 \pm 0$.' $08 \times 0$. $^{\prime} 86 \pm 0$." 07 on the sky). Although the galaxy is compact in imaging, spectroscopy reveals that it consists of at least two SF knots aligned not far from the line-of-sight (see Fig. 11) but separated by $130 \mathrm{~km} \mathrm{~s}^{-1}$ in velocity space. The GRB was coincident with the brighter, redshifted, southernmost knot; the fainter elongated feature likely corresponds to the second, blueshifted knot, hence it is in the foreground of the main SF knot. The three X-shooter spectra were taken with slightly different slit angles, hence covering slightly different percentages of the knots. The brightness of both afterglow and emission features allows us to perform a detailed study of the host using both gas emission and interstellar medium (ISM) properties from absorption in the afterglow spectrum.

\subsection{SED fit of the host galaxy}

In Table 5 we list the observed magnitudes from late-time imaging of the host galaxy from $u$ to $3.6 \mu \mathrm{m}$ bands using several different telescopes. We used LePhare (v. 2.2, Arnouts et al. 1999; Ilbert et al. 2006) to fit the optical-to-NIR SED of the host to a set of galaxy templates based on the models from Bruzual \& Charlot (2003). The SED shape is well-reproduced

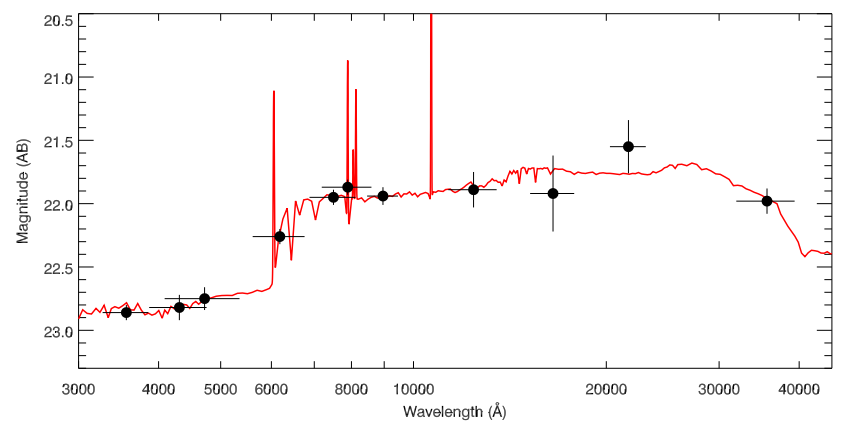

Fig. 8. Spectral energy distribution of GRB 100418A with the best fit from LePhare over-plotted in red.

$\left(\chi^{2} /\right.$ d.o.f. $\left.=2.3 / 8\right)$ by a galaxy template (see Fig. 8$)$ reddened by a Calzetti extinction law with $E(B-V)=0.1$ (Calzetti et al. $2000)$, an age of $203_{-82}^{+334} \mathrm{Myr}$, a mass of $\log \left(M_{*} / M_{\odot}\right)=9.20_{-0.13}^{+0.10}$, and a star formation rate of $\log \left(\mathrm{SFR} /\left(M_{\odot} \mathrm{yr}^{-1}\right)\right)=0.8_{-0.2}^{+0.3}$, implying a specific star formation rate of $\log \left(\mathrm{SSFR} \mathrm{yr}^{-1}\right)=-8.4_{-0.3}^{+0.4}$. The galaxy lies within the average value of GRB host masses at $z \sim 0.6$ but has a value for the SFR and hence the SSFR on the upper end of the distribution at that redshift (see e.g. Perley et al. 2016b; Vergani et al. 2015; Japelj et al. 2016).

\subsection{Absorbing gas along the line of sight}

The X-shooter spectra show absorption features commonly seen in GRB spectra, including Zn II, Cr II, Fe II, Mn II, Mg II, Mg I, Ti II, Ca II. In the first spectrum we also detect the Fe II* $\lambda 2396.36$ fine-structure line (see Table 6), which is thought to be excited by the GRB emission (Vreeswijk et al. 2007). This line, detected with an equivalent width of $0.13 \pm 0.2 \AA$ in the first epoch disappears at the time of the second spectrum, where we determine a $3-\sigma$ detection limit of $0.09 \AA$. This is consistent with the explanation of having been excited by the GRB, and allows us to unambiguously associate the GRB to the $z=0.6239$ redshift. In Table 6 we measured the combined absorption of each of the observed features in the first epoch, by calculating their equivalent widths. The line-strength diagram (following the prescription of de Ugarte Postigo et al. 2012b) in Fig. 9 shows that the absorption features are almost exactly on the average found for GRB afterglows, with the only exception being magnesium lines, which are slightly higher than average of the sample of de Ugarte Postigo et al. (2012b). We determine a line-strength parameter of LSP $=0.16 \pm 0.25$, which implies that the lines are consistent with the average of the sample (consistent with average value of $\operatorname{LSP}=0$ ) with a formal value that implies that they are stronger than those of $60 \%$ of the sample. We do not find a significant variation of the line strengths in the different epochs $(\mathrm{LSP}=0.04 \pm 0.32$ in the second epoch and LSP $=0.13 \pm 0.34$ in the third).

Using the first spectrum with the highest $\mathrm{S} / \mathrm{N}$ for the absorption lines we perform a Voigt-profile fitting of the absorption features, using FITLYMAN (Fontana \& Ballester 1995) in the MIDAS environment. The results of the fits are shown in Table 7. In the strongest features we identify up to five different velocity components at $v=0$ (component I), $-59,-173,-292$ and $-368 \mathrm{~km} \mathrm{~s}^{-1}$ (component V). Only the strongest absorption lines such as Fe II and $\mathrm{Mg}$ II $+\mathrm{Mg}$ I have absorption in all components (component $\mathrm{V}$ cannot be clearly identified in $\mathrm{Mg}$ I). Mn II, $\mathrm{Zn}$ II and $\mathrm{Cr}$ II are only present in components I and II, corresponding to the brighter SF region of the galaxy, although the velocities of $\mathrm{Zn}$ II and $\mathrm{Cr}$ II are 
Table 6. List of features identified in the spectra (detections $\gtrsim 3 \sigma$ ) along with their observer-frame equivalent widths.

\begin{tabular}{|c|c|c|c|}
\hline $\begin{array}{l}\lambda_{\text {obs }} \\
(\AA)\end{array}$ & $\begin{array}{c}\text { Feature } \\
(\AA)\end{array}$ & $z$ & $\begin{array}{c}\mathrm{EW}_{\mathrm{obs}} \\
(\AA)\end{array}$ \\
\hline 3290.12 & $\mathrm{Zn}$ пा 2026.14 & 0.6238 & $0.67 \pm 0.08$ \\
\hline 3338.59 & Cr п $\lambda 2056.26$ & 0.6236 & $0.26 \pm 0.05$ \\
\hline 3348.78 & Zn пл2062.66 & 0.6235 & $0.51 \pm 0.06$ \\
\hline 3354.91 & Crп $п 2066.16$ & 0.6237 & $0.19 \pm 0.04$ \\
\hline 3652.81 & Fe пा $\lambda 2249.88$ & 0.6236 & $0.40 \pm 0.05$ \\
\hline 3670.69 & Fe пा $\lambda 2260.78$ & 0.6236 & $0.38 \pm 0.04$ \\
\hline 3805.58 & Fe II $\lambda 2344.21$ & 0.6234 & $2.81 \pm 0.05$ \\
\hline 3854.97 & $\mathrm{Fe}$ пा $\lambda 2374.46$ & 0.6235 & $2.08 \pm 0.04$ \\
\hline 3868.05 & Fe пा $\lambda 2382.77$ & 0.6233 & $3.50 \pm 0.04$ \\
\hline 3891.45 & $\mathrm{Fe}_{\text {II }} * \lambda 2396.36$ & 0.6239 & $0.13 \pm 0.02$ \\
\hline 3934.57 & Са п $\lambda 3934.78$ & -0.0001 & $0.39 \pm 0.02$ \\
\hline 3969.04 & Са п $\lambda 3969.59$ & -0.0001 & $0.21 \pm 0.02$ \\
\hline 4183.91 & Mn пा $\lambda 2576.88$ & 0.6236 & $0.87 \pm 0.03$ \\
\hline 4199.24 & Fe п $\lambda 2586.65$ & 0.6234 & $3.02 \pm 0.03$ \\
\hline 4212.67 & Mn II $\lambda 2594.50$ & 0.6237 & $0.66 \pm 0.02$ \\
\hline 4221.02 & Fe пा $\lambda 2600.17$ & 0.6234 & $3.68 \pm 0.03$ \\
\hline 4231.96 & Mn пा $\lambda 2606.46$ & 0.6236 & $0.59 \pm 0.03$ \\
\hline 4538.95 & $\operatorname{Mg}_{\text {II } \lambda 2796.35}$ & 0.6232 & $5.53 \pm 0.04$ \\
\hline 4550.85 & $\operatorname{Mg}_{\text {II } \lambda 2803.53}$ & 0.6233 & $5.23 \pm 0.04$ \\
\hline 4631.90 & $\mathrm{Mg}_{\mathrm{I}} \lambda 2852.96$ & 0.6235 & $2.76 \pm 0.03$ \\
\hline 4990.98 & Ті пц $\lambda 073.88$ & 0.6237 & $0.12 \pm 0.02$ \\
\hline 5265.43 & Тi пा $\lambda 3242.93$ & 0.6237 & $0.17 \pm 0.02$ \\
\hline 5495.43 & Ті пा $\lambda 3384.74$ & 0.6236 & $0.15 \pm 0.02$ \\
\hline 5890.90 & Na 125891.58 & -0.0001 & $0.41 \pm 0.05$ \\
\hline 5897.12 & $\mathrm{Na} I \lambda 5897.56$ & -0.0001 & $0.23 \pm 0.04$ \\
\hline 6389.74 & Са пл 3934.78 & 0.6239 & $1.85 \pm 0.04$ \\
\hline 6446.29 & Са п $\lambda 3969.59$ & 0.6239 & $1.22 \pm 0.02$ \\
\hline
\end{tabular}

slightly shifted. Ca II has a somewhat different velocity structure than the other lines but also shows a weak component within the velocities corresponding to the second SF region.

The $\mathrm{Fe}_{\mathrm{II}}{ }^{*}$ fine-structure line is rather weak and only detected in the component at $v=0 \mathrm{~km} \mathrm{~s}^{-1}$ and disappears between the first and second epoch. This line was probably excited by the emission of the GRB via UV pumping or photo-excitation (see e.g. Vreeswijk et al. 2007, 2013; D'Elia et al. 2009). It also marks the closest velocity component to the GRB and hence determines by definition the redshift of the GRB and relative velocity $v=0 \mathrm{~km} \mathrm{~s}^{-1}$. We checked the different epochs for any variation in the strength of the resonant absorption lines but do not find any evidence. The absorbing column density of the disappearing $\mathrm{Fe}_{\mathrm{II}}{ }^{*}$ fine-structure line would show up as additional absorption in the corresponding resonant transition. However, the absorption in Fe II in this component is two dex higher than the $\mathrm{Fe}_{\text {II }}^{*}$ column density, is blended with component II, and even in the weakest transition of Fe II ( $\lambda 2260 \AA)$ mildly saturated. Hence making the uncertainty in the fit larger than any additional absorption in component I of Fe II (adding the absorbing column of $\mathrm{Fe}_{\mathrm{II}}{ }^{*}$ onto component I would add $<0.01$ dex to the column density of Fe II). The data of the third epoch have too low $\mathrm{S} / \mathrm{N}$ for any further constraints due to the decrease of the afterglow continuum.

\subsection{Host galaxy emission lines}

Already in the first spectrum, still dominated by the afterglow, the emission lines from the host galaxy are well-detected.
However, in the following analysis we use the spectrum of the third epoch at $2.5 \mathrm{~d}$ post-GRB, unless otherwise mentioned, which has higher $\mathrm{S} / \mathrm{N}$ in the emission lines due to a lower afterglow contribution and a better alignment of the slit comprising most of the flux of the host galaxy (see Fig. 11). We detect at least 17 emission lines as detailed in Table 8, including Balmer and Paschen lines, [O II $],\left[\mathrm{O}_{\mathrm{III}}\right],\left[\mathrm{Ne}_{\mathrm{II}}\right],\left[\mathrm{N}_{\mathrm{II}}\right],\left[\mathrm{S}_{\mathrm{II}}\right]$, [S $\left.\mathrm{S}_{\mathrm{III}}\right]$, and [He I]. These features all show two main velocity components which, for the higher $\mathrm{S} / \mathrm{N}$ lines, can be separated into further subcomponents, which we will detail in Sect. 5.6 Table 8 displays the measurement of the emission lines observed in the spectrum. For each of the two components, we show the full-width halfmaximum, central wavelength and flux resulting from a double Gaussian deblend procedure. The last two columns show the central wavelength and flux of the combined lines, measured without assuming any line profile.

Apart from the $\mathrm{He}_{\mathrm{I}} \lambda 10833$ line we do not detect any other transitions of He I such as the lines at $\lambda_{\text {rest }}=3819,4026,4471$, $4922,5019,5876,6678$ or $7065 \AA$ nor ionised He II at $\lambda 3204$, 4686 or $10126 \AA$. The He I $\lambda 10833$ emission line is the transition from the He-triplet state $2^{3} \mathrm{P}_{0}(1 s 2 p)$ to $2^{3} \mathrm{~S}(1 s 2 s)$ and can be populated either by collisions from the $2^{3} \mathrm{~S}$ or after recombination in the downward cascade from a higher excited state e.g. via emission of $\lambda \lambda 4471,5876$ or $7065 \AA$ photons, none of which we detect (for a Grotrian diagram see e.g. Benjamin et al. 1999). Due to the additional population by collisions, the ratio of e.g. He I 10833 to 4471 increases at higher densities and temperatures and can vary between a few and more than $40 \mathrm{~K}$ (Benjamin et al. 1999). We determine flux limits for He I 4771 and 7065 (the 5876 line is in the middle of a skyline) of $<1.01 \times 10^{-17}$ and $<1.06 \times 10^{-16} \mathrm{erg} \mathrm{s}^{-1} \mathrm{~cm}^{-2}$, respectively. The tighter limit for He 4771 together with the flux measured for combined He I 10833 (see Table 8) gives limits on temperatures and densities less than $5000 \mathrm{~K}$ and $n_{\mathrm{e}}=10^{2} \mathrm{~cm}^{-2}$ according to the values tabulated in Benjamin et al. (1999).

$\mathrm{He}_{\mathrm{I}}$ is only visible in emission a few Myr after the onset of the starburst which allows for a rather accurate age determination of the stellar population (González Delgado 1999). He I has been detected in a few nearby GRB hosts (GRB 060218, Wiersema et al. 2007; GRB 031203, Margutti et al. 2007; Guseva et al. 2011; GRB 100316D, Izzo et al. 2017; and possibly GRB 120422A, Schulze et al. 2014) but only in the VIS regime due to lack of high $\mathrm{S} / \mathrm{N}$ infrared spectra with the exception of the X-shooter spectrum of GRB 031203 (Guseva et al. 2011). However, the dependence of the $\lambda 10833$ transition on temperature and density (due to population by collisions) makes it very little suited as an age indicator for the stellar population in contrast to the VIS transitions of He I. He II, which we do not detect, requires not only a young population but also a strongly ionising radiation field and has been observed to be stronger at low metallicities (Schaerer 2003). Some have associated the emission of narrow (nebular) He II emission to Wolf-Rayet (WR) stars but they are not always spatially coincident and WR stars (Kehrig et al. 2015) are much rarer at low metallicities. For He II 3204 and 10126 we derive limits of $<1.53 \times 10^{-17}$ and $<9.3 \times 10^{-17} \mathrm{erg} \mathrm{s}^{-1} \mathrm{~cm}^{-2}$, respectively, He II $\lambda 4686$, unfortunately, is in the atmospheric A band, so no useful limits can be derived. A few other GRB hosts show He II emission (see e.g. Han et al. 2010) but at luminosities lower than inferred from our detection limit. As the afterglow was still bright during the last X-shooter epoch, the non-detection is not surprising nor constraining.

The electron density is commonly derived using the ratio of the $\left[\mathrm{S}_{\mathrm{II}}\right] \lambda \lambda 6716,6732$ or the $\left[\mathrm{O}_{\mathrm{II}}\right] \lambda \lambda 3727,3729$ doublets. 


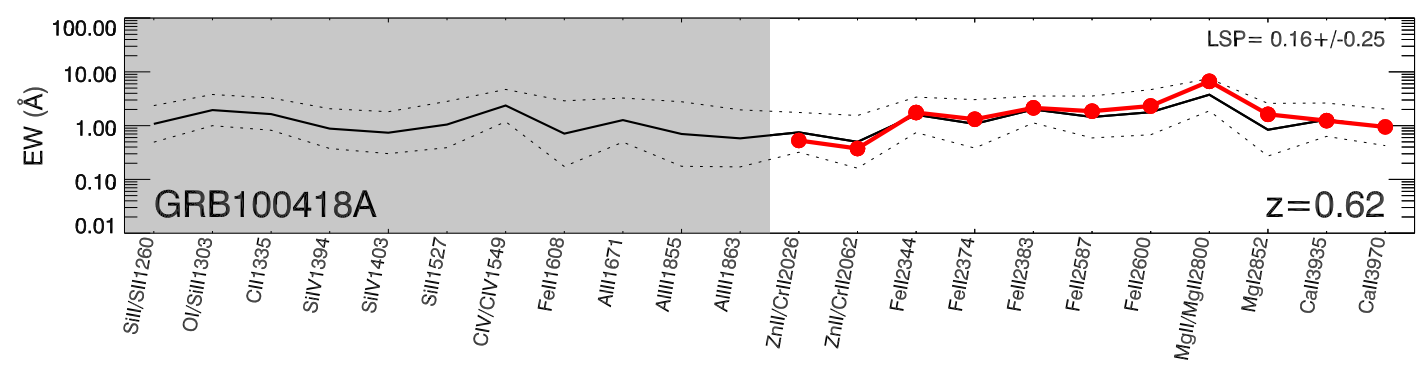

Fig. 9. Line strength diagram of the afterglow of GRB 100418A, following the prescription of de Ugarte Postigo et al. (2012b). This figure compares the strength of the spectral features of GRB 100418A (in red) with the average values of a sample of 69 spectra (in black).

Table 7. List of features identified in the first X-shooter spectrum (detections $\gtrsim 3 \sigma$ ).

\begin{tabular}{|c|c|c|c|c|c|c|c|c|c|c|c|}
\hline \multirow[b]{2}{*}{ Ion } & \multirow[b]{2}{*}{$\begin{array}{c}\text { Transitions } \\
(\AA)\end{array}$} & \multicolumn{2}{|c|}{ Component I } & \multicolumn{2}{|c|}{ Component II } & \multicolumn{2}{|c|}{ Component III } & \multicolumn{2}{|c|}{ Component IV } & \multicolumn{2}{|c|}{ Component V } \\
\hline & & $\begin{array}{c}\text { vel. } \\
\mathrm{km} \mathrm{s}^{-1}\end{array}$ & $\begin{array}{l}\log \mathrm{N} \\
\mathrm{cm}^{-2}\end{array}$ & $\begin{array}{c}\text { vel. } \\
\mathrm{km} \mathrm{s}^{-1}\end{array}$ & $\begin{array}{l}\log \mathrm{N} \\
\mathrm{cm}^{-2}\end{array}$ & $\begin{array}{c}\text { vel. } \\
\mathrm{km} \mathrm{s}^{-1}\end{array}$ & $\begin{array}{l}\log \mathrm{N} \\
\mathrm{cm}^{-2}\end{array}$ & $\begin{array}{c}\text { vel. } \\
\mathrm{km} \mathrm{s}^{-1}\end{array}$ & $\begin{array}{l}\log \mathrm{N} \\
\mathrm{cm}^{-2}\end{array}$ & $\begin{array}{c}\text { vel. } \\
\mathrm{km} \mathrm{s}^{-1}\end{array}$ & $\begin{array}{l}\log \mathrm{N} \\
\mathrm{cm}^{-2}\end{array}$ \\
\hline $\mathrm{Zn}$ II & 2026,2062 & +0 & $13.31 \pm 0.06$ & -59 & $13.05 \pm 0.21$ & -173 & $12.87 \pm 0.12$ & - & - & - & - \\
\hline Cr II & 056,2066 & +0 & $13.75 \pm 0.07$ & -59 & $13.53 \pm 0.14$ & -173 & $13.44 \pm 0.10$ & - & - & - & - \\
\hline $\mathrm{Fe}_{\text {II }}$ & $\begin{array}{c}2249,2260,2344,2374 \\
2382,2586,2600\end{array}$ & +0 & $15.19 \pm 0.06$ & -59 & $15.35 \pm 0.05$ & -173 & $14.49 \pm 0.03$ & -292 & $13.46 \pm 0.01$ & -368 & $12.96 \pm 0.06$ \\
\hline $\mathrm{Fe}$ II & 2396 & +0 & $12.86 \pm 0.05$ & - & - & - & - & - & - & - & - \\
\hline Mn II & $2576,2594,2606$ & +0 & $13.17 \pm 0.02$ & -59 & $13.22 \pm 0.02$ & -173 & $12.54 \pm 0.07$ & - & - & - & - \\
\hline $\mathrm{Mg}_{\text {II }}$ & 2796, 2803 & +0 & $16.00 \pm 0.17$ & -59 & $16.61 \pm 0.17$ & -173 & $14.71 \pm 0.15$ & -292 & $13.78 \pm 0.08$ & -368 & $13.06 \pm 0.03$ \\
\hline $\mathrm{Mg}_{\mathrm{I}}$ & 2852 & +0 & $13.10 \pm 0.02$ & -59 & $12.98 \pm 0.01$ & -173 & $12.58 \pm 0.01$ & -292 & $12.20 \pm 0.0 .3$ & - & - \\
\hline Ti II & $3073,3242,3384$ & -16 & $12.76 \pm 0.47$ & -76 & $12.73 \pm 0.29$ & - & - & - & - & - & - \\
\hline Ca II & 3934, 3969 & +40 & $13.38 \pm 0.03$ & +8 & $15.19 \pm 0.22$ & -55 & $12.89 \pm 1.00$ & -154 & $12.21 \pm 0.03$ & - & - \\
\hline
\end{tabular}

Since the $\left[\mathrm{O}_{\mathrm{II}}\right]$ is partially blended due to the extended components, we derive electron temperatures from the [S II] doublet using a recent recalibration of Osterbrock \& Ferland (2006) by Proxauf et al. (2014). At $T_{\mathrm{e}}=10,000 \mathrm{~K}$, the ratios of $I_{6716} / I_{6732}$ result in electron densities between $n_{\mathrm{e}} \sim 10^{3}-10^{2} \mathrm{~cm}^{-2}$ for the components and the entire host. For other temperatures, the electron density only changes by a small value. The result is consistent with the limit derived from the ratio of He I 10830/4471 (see above).

[S III] $\lambda \lambda 9069,9532$ can be used as indicator for excitation of the ISM and as a tracer to distinguish between HII regions and AGN activity (Diaz et al. 1985). We do not detect the 19069 line but the [S III] doublet has a fixed ratio of 2.486 (Aller 1984). The host of GRB 100418A lies on the edge of the region in the plot occupied by HII regions in Diaz et al. (1985) as the fluxes of [S III] and [S II] are comparable in value. For HII regions and starbursts, the flux of the [S III] $\lambda \lambda 9069,9532$ is usually higher than the flux of the [S II] doublet. This could either mean a low ionisation or an enhanced [S II] flux, indicative of shocked regions or AGN activity, which seems unlikely when placing the galaxy in the BPT diagram (see Fig. 12). We can also derive a sulphur abundance from $\mathrm{S}_{23}=\left([\mathrm{S}\right.$ III $] \lambda \lambda 9069,9532+\left[\mathrm{S}_{\text {II }}\right] \lambda \lambda 6017$, $6732) / \mathrm{H} \beta$ adopting the calibration $12+\log (\mathrm{S} / \mathrm{H})=6.540+2.071$ $\log \mathrm{S}_{23}+0.348 \log \mathrm{S}_{23}^{2}$ from Pérez-Montero et al. (2006) and obtain $12+\log (\mathrm{S} / \mathrm{O}) \sim 7.3$, a relatively high value but consistent with the high oxygen abundance in the host of GRB 100418A as the ratio S/O has been shown not to vary with metallicity, hence $\mathrm{S}$ and $\mathrm{O}$ are tightly related (see e.g. Kehrig et al. 2006).

[Ne III] is usually tightly related to [O III] $\lambda 5008$ as its isoelectronic equivalent, however, [Ne III] has a somewhat higher ionisation potential and excitation energy. Also, $\mathrm{Ne}$ and $\mathrm{O}$ are both $\alpha$ elements produced in Type II SNe and hence related to massive star formation. In both components and the total line flux, we measure $\log ([\mathrm{Ne} \mathrm{III}] /[\mathrm{O} I \mathrm{III}])=-1.09,-1.15$ and -1.13 , consistent with values around -1.1 found for local SDSS galaxies and independent of $T_{\mathrm{e}}$, stellar mass or metallicity (Izotov et al. 2006; Zeimann et al. 2015). The ratio of $\log ([\mathrm{Ne} \text { III }] /[\mathrm{O} \text { III }])_{\text {tot }}=-1.2$ is also consistent with the values found for nearby galaxies and the stellar mass derived in Sect. 5.1 of $\log M^{*}=9.2 M_{\odot}$. At redshifts $z>1$, both ratios are enhanced by $\sim 0.2$ dex, the reason for which is still debated, possibilities being Ne enhancement from WR stars, higher densities or higher oxygen depletion (Zeimann et al. 2015).

\subsection{Host galaxy extinction and SFR}

To derive the properties of the host galaxy we analysed the two main emission components, their sum, as well as the combined spectrum. The fluxes of the individual lines were determined by fitting them with Gaussian functions, whereas the measurements for the combined spectrum were obtained by measuring the flux above the continuum without assuming any line shape. Hence, the integrated flux gives us more accurate measurements but does not allow us to deblend the different components within the host. We estimated the interstellar extinction of the host galaxy from the Balmer decrement, adopting the Calzetti et al. (2000) attenuation curve with $R_{V}=4.05$ which is commonly observed in starburst galaxies. We assume a standard recombination model for SF galaxies and the Case B of HI recombination lines (Osterbrock \& Ferland 2006) corresponding to an electron temperature of $T=10^{4} \mathrm{~K}$ and density of $n_{\mathrm{e}}=10^{2} \mathrm{~cm}^{-3}$. This results in an extinction of $E(B-V)_{1,2 \text {, com }}=0.24 \pm 0.09$, $0.08 \pm 0.06,0.16 \pm 0.03 \mathrm{mag}$, for the first, the second component and combined flux including the entire galaxy, respectively. 
Table 8. List of emission lines and their fluxes measured in the different spectra.

\begin{tabular}{|c|c|c|c|c|}
\hline $\begin{array}{c}\text { Line } \\
\AA\end{array}$ & $\begin{array}{c}\text { Flux }_{1} \\
\left(10^{-17} \mathrm{erg} \mathrm{s}^{-1} \mathrm{~cm}^{-2}\right)\end{array}$ & $\begin{array}{c}\text { Flux }_{2} \\
\left(10^{-17} \mathrm{erg} \mathrm{s}^{-1} \mathrm{~cm}^{-2}\right) \\
\end{array}$ & $\begin{array}{c}\text { Flux }_{1+2} \\
\left(10^{-17} \mathrm{erg} \mathrm{s}^{-1} \mathrm{~cm}^{-2}\right)\end{array}$ & $\begin{array}{c}\text { Flux }_{\text {Comb }} \\
\left(10^{-17} \mathrm{erg} \mathrm{s}^{-1} \mathrm{~cm}^{-2}\right)\end{array}$ \\
\hline \multicolumn{5}{|c|}{ EPOCH 1} \\
\hline$[\mathrm{O}$ Іा] $] 3727.09$ & $6.04 \pm 0.55$ & $9.61 \pm 0.79$ & $15.65 \pm 0.96$ & $41.89 \pm 0.95$ \\
\hline [О п] $] \lambda 3729.87$ & $9.11 \pm 0.64$ & $14.78 \pm 0.80$ & $23.89 \pm 1.02$ & Blend \\
\hline$[\mathrm{Ne}$ пा] $] 3870.87$ & - & - & - & - \\
\hline H $6 \lambda 3890.15$ & $0.23 \pm 0.50$ & $1.78 \pm 0.63$ & $2.01 \pm 0.80$ & $2.48 \pm 0.81$ \\
\hline Н $5 \lambda 3971.20$ & - & - & - & - \\
\hline Н $4 \lambda 4102.89$ & $0.85 \pm 0.43$ & $1.90 \pm 0.54$ & $2.74 \pm 0.69$ & $2.96 \pm 0.60$ \\
\hline Н $3 \lambda 4341.68$ & $1.77 \pm 0.41$ & $3.37 \pm 0.52$ & $5.14 \pm 0.66$ & $5.16 \pm 0.93$ \\
\hline $\mathrm{H} \beta \lambda 4862.68$ & $4.46 \pm 0.46$ & $9.21 \pm 0.61$ & $13.67 \pm 0.76$ & $14.30 \pm 0.70$ \\
\hline$\left[\mathrm{O}_{\text {III }}\right] \lambda 4960.30$ & $3.35 \pm 0.42$ & $7.46 \pm 0.50$ & $10.81 \pm 0.65$ & $11.09 \pm 0.72$ \\
\hline [O III] $] \lambda 5008.24$ & $10.09 \pm 0.40$ & $20.21 \pm 0.62$ & $31.12 \pm 0.62$ & $32.76 \pm 0.69$ \\
\hline $\mathrm{H} \alpha \lambda 6564.61$ & $14.40 \pm 1.43$ & $25.07 \pm 2.02$ & $39.47 \pm 2.48$ & $41.19 \pm 1.25$ \\
\hline$\left[\mathrm{N}_{\text {II }}\right] \lambda 6585.28$ & $1.22 \pm 1.58$ & $2.35 \pm 2.44$ & $3.57 \pm 2.91$ & $(2.76 \pm 1.5)$ \\
\hline$[\mathrm{S}$ ІІ $] \lambda 6718.29$ & $1.34 \pm 1.47$ & $3.45 \pm 1.57$ & $4.80 \pm 2.16$ & $5.01 \pm 1.33$ \\
\hline$\left[\mathrm{S}_{\text {II }}\right] \lambda 6732.67$ & $0.94 \pm 1.34$ & $1.34 \pm 1.71$ & $2.27 \pm 2.16$ & $5.77 \pm 1.26$ \\
\hline$\left[\mathrm{S}_{\mathrm{III}}\right] \lambda 9533.20$ & $2.52 \pm 0.82$ & $4.88 \pm 0.72$ & $7.40 \pm 1.08$ & $6.57 \pm 1.28$ \\
\hline Не г $\lambda 10833.3$ & - & - & - & - \\
\hline $\operatorname{Pa} \gamma \lambda 10941.10$ & - & - & - & - \\
\hline \multicolumn{5}{|c|}{ EPOCH 2} \\
\hline$[\mathrm{O}$ п] $] \lambda 3727.09$ & $6.94 \pm 0.52$ & $14.28 \pm 0.78$ & $21.22 \pm 0.94$ & $53.32 \pm 1.24$ \\
\hline$[$ О п] $] \lambda 3729.87$ & $9.87 \pm 0.55$ & $19.03 \pm 0.79$ & $28.9 \pm 0.96$ & Blend \\
\hline$[\mathrm{Ne}$ II $] \lambda 3870.87$ & $1.31 \pm 0.41$ & $2.06 \pm 0.64$ & $3.37 \pm 0.76$ & $3.58 \pm 0.91$ \\
\hline H $6 \lambda 3890.15$ & $1.27 \pm 0.41$ & $1.54 \pm 0.58$ & $2.81 \pm 0.71$ & $3.24 \pm 0.41$ \\
\hline Н $5 \lambda 3971.20$ & - (Sky line) & $2.09 \pm 0.69$ & $>2.09 \pm 0.81$ & $2.16 \pm 0.33$ \\
\hline Н $4 \lambda 4102.89$ & $1.13 \pm 0.40$ & $3.09 \pm 0.56$ & $4.23 \pm 0.69$ & $4.82 \pm 0.57$ \\
\hline Н $3 \lambda 4341.68$ & $1.96 \pm 0.35$ & $4.68 \pm 0.49$ & $6.63 \pm 0.60$ & $7.20 \pm 0.30$ \\
\hline $\mathrm{H} \beta \lambda 4862.68$ & $5.10 \pm 0.41$ & $11.58 \pm 0.54$ & $16.68 \pm 0.68$ & $17.35 \pm 0.31$ \\
\hline [O III] $] \lambda 4960.30$ & $4.18 \pm 0.34$ & $9.19 \pm 0.48$ & $13.37 \pm 0.59$ & $14.99 \pm 0.45$ \\
\hline$\left[\mathrm{O}_{\mathrm{III}}\right] \lambda 5008.24$ & $13.36 \pm 0.33$ & $28.27 \pm 0.53$ & $41.62 \pm 0.62$ & $41.79 \pm 1.06$ \\
\hline $\mathrm{H} \alpha \lambda 6564.61$ & $14.75 \pm 1.65$ & $31.37 \pm 1.65$ & $46.12 \pm 2.87$ & $52.64 \pm 1.96$ \\
\hline$[\mathrm{N}$ пा] 6585.28 & $0.69 \pm 1.86$ & $1.64 \pm 2.95$ & $2.33 \pm 3.49$ & $4.23 \pm 1.02$ \\
\hline$[\mathrm{S}$ пI $] \lambda 6718.29$ & $3.08 \pm 1.68$ & $5.00 \pm 1.80$ & $8.07 \pm 2.46$ & $10.75 \pm 2.35$ \\
\hline$[\mathrm{S}$ II $] \lambda 6732.67$ & $1.50 \pm 1.54$ & $2.18 \pm 1.99$ & $3.68 \pm 2.52$ & $6.41 \pm 1.05$ \\
\hline$\left[\mathrm{S}_{\text {III }}\right] \lambda 9533.20$ & $3.13 \pm 0.75$ & $5.83 \pm 0.70$ & $8.97 \pm 1.03$ & $8.64 \pm 1.23$ \\
\hline Не І $\lambda 10833.3$ & $1.19 \pm 0.55$ & $1.03 \pm 0.92$ & $2.23 \pm 1.07$ & $1.69 \pm 1.00$ \\
\hline $\mathrm{Pa} \gamma \lambda 10941.10$ & - & - & - & - \\
\hline \multicolumn{5}{|c|}{ EPOCH 3} \\
\hline$[\mathrm{O}$ пा $] \lambda 3727.09$ & $7.26 \pm 0.43$ & $12.96 \pm 0.58$ & $20.22 \pm 0.72$ & $49.74 \pm 0.70$ \\
\hline$[\mathrm{O}$ п] $] \lambda 3729.87$ & $10.21 \pm 0.52$ & $17.92 \pm 0.66$ & $28.13 \pm 0.84$ & Blend \\
\hline$[\mathrm{Ne}$ II] $] 3870.87$ & $1.23 \pm 0.33$ & $2.17 \pm 0.47$ & $3.41 \pm 0.57$ & $3.28 \pm 0.42$ \\
\hline H $6 \lambda 3890.15$ & $0.24 \pm 0.37$ & $1.53 \pm 0.47$ & $1.77 \pm 0.60$ & $1.94 \pm 0.37$ \\
\hline H $5 \lambda 3971.20$ & $0.68 \pm 0.39$ & $1.22 \pm 0.50$ & $1.90 \pm 0.64$ & $2.02 \pm 0.37$ \\
\hline H $4 \lambda 4102.89$ & $0.84 \pm 0.32$ & $2.32 \pm 0.46$ & $3.16 \pm 0.56$ & $3.11 \pm 0.48$ \\
\hline Н $3 \lambda 4341.68$ & $1.87 \pm 0.28$ & $4.35 \pm 0.41$ & $6.22 \pm 0.50$ & $6.10 \pm 0.72$ \\
\hline $\mathrm{H} \beta \lambda 4862.68$ & $5.22 \pm 0.37$ & $10.79 \pm 0.49$ & $16.01 \pm 0.61$ & $16.37 \pm 0.46$ \\
\hline [O III] $] \lambda 4960.30$ & $3.99 \pm 0.32$ & $8.88 \pm 0.38$ & $12.87 \pm 0.50$ & $13.66 \pm 0.32$ \\
\hline$\left[\mathrm{O}_{\text {III }}\right] \lambda 5008.24$ & $12.51 \pm 0.30$ & $26.39 \pm 0.44$ & $38.90 \pm 0.53$ & $39.25 \pm 0.39$ \\
\hline $\mathrm{H} \alpha \lambda 6564.61$ & $19.75 \pm 1.65$ & $34.06 \pm 1.94$ & $53.82 \pm 2.55$ & $56.43 \pm 1.00$ \\
\hline$\left[\mathrm{N}_{\mathrm{II}}\right] \lambda 6585.28$ & $3.08 \pm 1.93$ & $5.91 \pm 2.42$ & $8.98 \pm 3.10$ & $6.03 \pm 1.19$ \\
\hline$[\mathrm{S}$ пI $] \lambda 6718.29$ & $1.99 \pm 1.28$ & $4.53 \pm 1.47$ & $6.52 \pm 1.95$ & $7.70 \pm 1.01$ \\
\hline$\left[\mathrm{S}_{\text {II }}\right] \lambda 6732.67$ & $2.64 \pm 1.53$ & $3.74 \pm 1.67$ & $6.38 \pm 2.27$ & $6.75 \pm 1.41$ \\
\hline$\left[\mathrm{S}_{\text {III }}\right] \lambda 9533.20$ & $2.26 \pm 0.80$ & $5.34 \pm 0.68$ & $7.60 \pm 1.05$ & $8.33 \pm 1.01$ \\
\hline Не г $\lambda 10833.3$ & $1.49 \pm 0.52$ & $2.10 \pm 0.93$ & $3.59 \pm 1.07$ & $4.11 \pm 1.02$ \\
\hline Ра $\gamma \lambda 10941.10$ & $0.47 \pm 0.87$ & $1.26 \pm 0.97$ & $1.73 \pm 1.30$ & $2.77 \pm 1.00$ \\
\hline
\end{tabular}

Notes. Fluxes are corrected for Galactic extinction and normalised to the photometry. No intrinsic extinction corrections have been applied.

We adopt the extinction value determined from the 3 rd epoch for all spectra, since the higher $\mathrm{S} / \mathrm{N}$ ratio gives us a better accuracy.

We use the luminosity of $\mathrm{H} \alpha$ and the $\mathrm{O}$ II $\lambda 3727,3729$ nebular lines as the tracers of SFR by converting the luminosities of the two lines following the Kennicutt et al. (1994) relations. For this calculation we assume a Salpeter initial mass function
(IMF) $\left(\mathrm{N}(\mathrm{m}) \propto \mathrm{m}^{-2.35}\right)$ with masses of $0.1-100 M_{\odot}$, solar metallicity, and continuous star formation. The $\mathrm{H} \alpha$ recombination line directly couples the nebular emission with massive star formation, but has some significant limitations: The method is especially sensitive to extinction and IMF uncertainties and works under the assumption that the ionised gas is a tracer of all the 
Table 9. Properties for the two main host regions and the entire host complex derived from different emission lines in the spectra of the third epoch.

\begin{tabular}{|c|c|c|c|}
\hline Property & Region 1 & Region 2 & Combined host \\
\hline SFR H $\alpha\left(M_{\odot} \mathrm{yr}^{-1}\right)$ & $3.8 \pm 0.3$ & $8.3 \pm 0.5$ & $12.2 \pm 0.6$ \\
\hline$E(B-V)(\mathrm{mag})$ & $0.24 \pm 0.09$ & $0.08 \pm 0.06$ & $0.16 \pm 0.03$ \\
\hline $12+\log (\mathrm{O} / \mathrm{H})$ & $8.53_{-0.13}^{+0.14}$ & $8.63_{-0.05}^{+0.05}$ & $8.545_{-0.015}^{+0.016}$ \\
\hline$n_{\mathrm{e}}\left(\mathrm{cm}^{-2}\right)$ & $10^{-0.13}$ & $1.02 \times 10^{2}$ & $1.08 \times 10^{2}$ \\
\hline$S_{23}$ & $1.75 \pm 0.28$ & $2.11 \pm 0.16$ & $1.82 \pm 0.14$ \\
\hline $\log \left[\mathrm{N}_{\mathrm{II}}\right] / \mathrm{H} \alpha$ & $-1.07 \pm 0.27$ & $-1.03 \pm 0.18$ & $-1.17 \pm 0.15$ \\
\hline $\log \left[\mathrm{S}_{\mathrm{II}}\right] / \mathrm{H} \alpha$ & $-0.63 \pm 0.20$ & $-0.61 \pm 0.09$ & $-0.59 \pm 0.08$ \\
\hline $\log \left[\mathrm{O}_{\mathrm{III}}\right] / \mathrm{H} \beta$ & $0.35 \pm 0.03$ & $0.43 \pm 0.03$ & $0.36 \pm 0.02$ \\
\hline $\log \mathrm{N} / \mathrm{O}$ & $-0.37 \pm 0.27$ & $-0.34 \pm 0.18$ & $-0.35 \pm 0.15$ \\
\hline
\end{tabular}

Notes. For details, we refer to Sects. 5.3 and 5.4. The afterglow was coincident with Region 2.

massive star formation. For the escape fraction of ionising radiation an upper limit of 3\% has been established (Leitherer et al. 1995; Tanvir et al. 2018). The properties of galaxies derived using a Salpeter Initial Mass Function (IMF) slope are consistent with the stellar population observed in nearby galaxies (Massey 1998) so we expect that the chosen IMF slope does not affect the results significantly.

In contrast, the forbidden [O II] $\lambda 3727,3729$ doublet is not directly coupled to the ionising flux but is sensitive to the gas abundance and ionisation state and the excitation of [O II] can be calibrated empirically through $\mathrm{H} \alpha$. The mean ratio of $[\mathrm{O}$ II $] \lambda 3727 / \mathrm{H} \alpha$ in an individual galaxy varies between 0.5 and 1.0 dex reflecting the differences between samples used to derive the SFR calibration from [O II] $\lambda 3727$ (Gallagher et al. 1989; Kennicutt 1992). Here we adopt the average calibration. The values in both SFRs reported below have been calculated after correcting for extinction. We then obtain a SFR of $12.2 \pm 0.6 M_{\odot} \mathrm{yr}^{-1}$ from $\mathrm{H} \alpha$ and $6.085 M_{\odot} \mathrm{yr}^{-1}$ from [O II] for the entire host galaxy. This discrepancy is probably due to an overcorrection of the extinction in the $[\mathrm{OII}]$ line, which being in the blue is more affected by extinction uncertainty. Because of this, we use the $\mathrm{H} \alpha$ value as the reference one. We note that this SFR is consistent with the value determined from the SED fit, but significantly above the one determined by Krühler et al. (2015), who estimate $\mathrm{SFR}=4.2_{-0.8}^{+1.0} M_{\odot} \mathrm{yr}^{-1}$. The difference with the values from Krühler et al. (2015), who used the same data set are due to a different extraction of the spectrum and matched photometry flux calibration correction, since the extinction is equal in both cases.

\subsection{Host galaxy abundances and metallicity}

In order to estimate metallicities $(\mathrm{Z})$ we used the Python code pyMCZ (Bianco et al. 2016) that determines oxygen abundance using strong emission-lines standard metallicity diagnostics. The code derives the statistical oxygen abundance confidence region via Monte Carlo simulations. Various emission-line ratios are used in up to 15 theoretical/empirical/combined metallicity calibrations. For the metallicity analysis of the host we used the following strong emission-line fluxes: [O II] $] 3727 / 3729$, $\mathrm{H} \beta,\left[\mathrm{O}_{\text {III }}\right] \lambda \lambda 4959,5007, \mathrm{H} \alpha,\left[\mathrm{N}_{\text {II }}\right] \lambda 6584,\left[\mathrm{~S}_{\text {II }}\right] \lambda \lambda 6717,6731$, [S III] $\lambda^{2}$ 9532. [O III] $\lambda 4363$ is not detected in the spectra, likely due to the relatively high metallicity of the system (see below). The values of line fluxes were corrected for both Galactic and intrinsic reddening before calculating the metallicities. The final

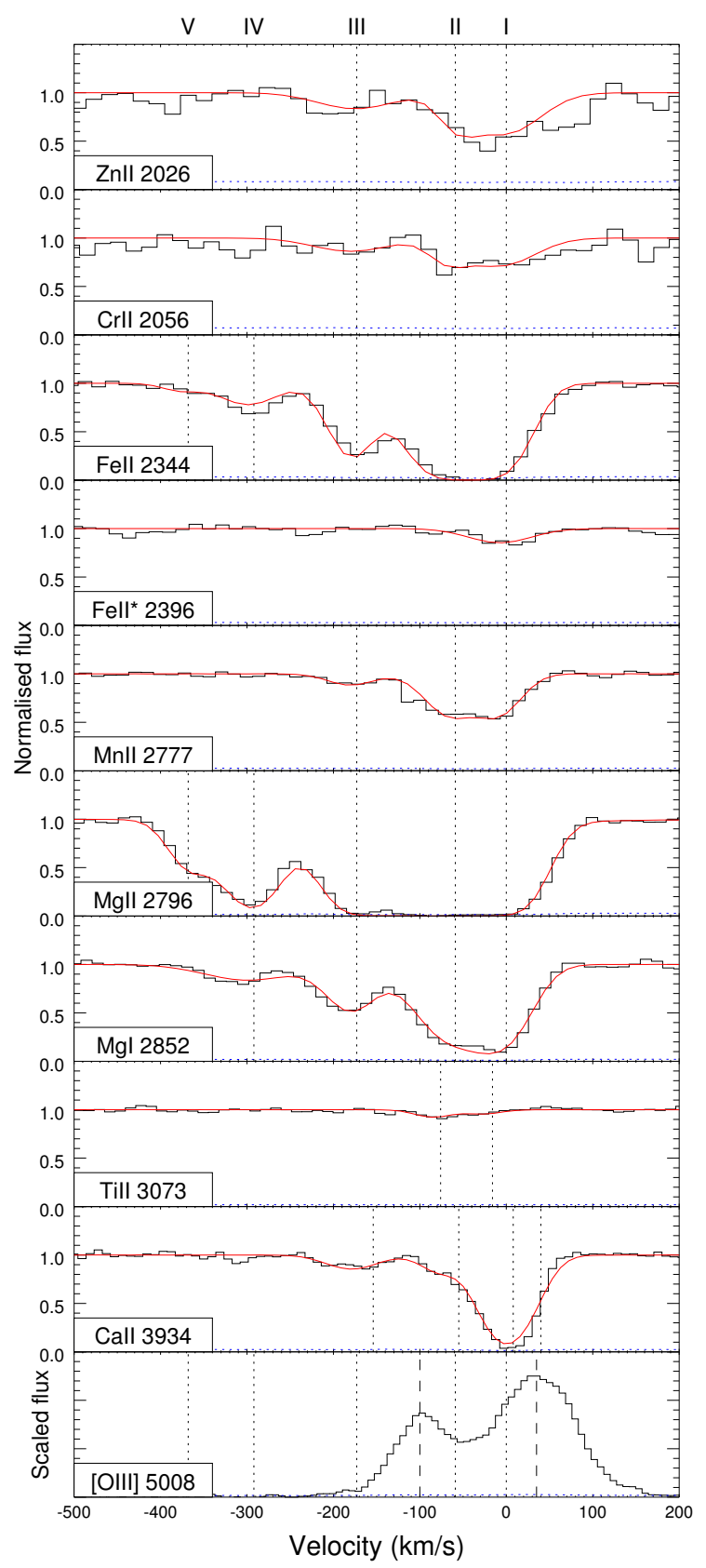

Fig. 10. Voigt profile fitting of several absorption features, and the [O III] $\lambda 5008$ emission in the lower panel. The five main absorption components are indicated with roman numbers at the top. In the bottom panel we have also indicated the two main emission components with dashed lines.

adopted metallicity for the three different components are listed in Table 9. The full set of metallicity measurements using different calibrators can be found in Table A.3 in the appendix. For a detailed discussion on the different calibrators and their abbreviations below we refer to the references in the table.

There has been a long-standing debate on which diagnostic should be adopted to have the best metallicity estimate. It is also known that there are systematic offsets between different calibrators (see e.g. Kewley \& Ellison 2008). In order to properly compare the oxygen abundances provided by different methods using different sets of emission lines we used the metallicity conversion determined by Kewley \& Ellison (2008) 


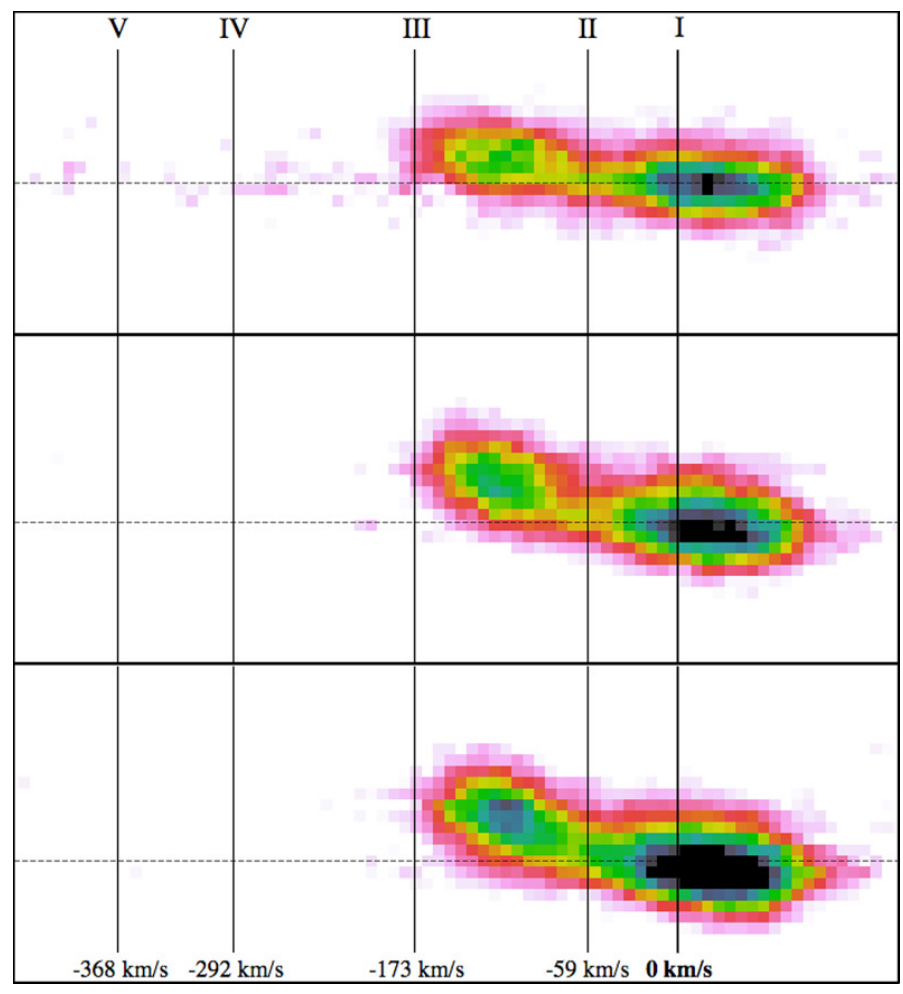

Fig. 11. Section of the $2 \mathrm{D}$ spectra showing the $\left[\mathrm{O}_{\mathrm{III}}\right] \lambda 5008.24$ emission line, with continuum subtracted, for each of the three spectral epochs (from top to bottom panels). The spatial axis is vertical, while the spectral dispersion is horizontal. The northernmost part of the slit is to the top (see Fig. 7 for each slit orientation). The dashed line marks the position of the GRB afterglow in the spatial direction. Horizontal lines indicate the relative velocities of the 5 main absorption components, some of which are related to the emission features.

which allows the different metallicities to be converted into the same base calibration (in this case Kewley \& Dopita (2002), henceforth KD02). We present the converted metallicities in the bottom part of Table A.3 for each epoch. All but one method give results within 0.1 dex after converting to the base metallicity. The only exception is the M91 calibration where we use the $\left[\mathrm{N}_{\text {II }}\right] \lambda 6584 /\left[\mathrm{O}_{\mathrm{II}}\right] \lambda 3727$ line ratio in order to break the R23 degeneracy. This method directly depends on the very weak $[\mathrm{N}$ II] $\lambda 6584$ line and indeed, the weaker this line is, independent of the value of the $\left[\mathrm{N}_{\mathrm{II}}\right] \lambda 6584 /\left[\mathrm{O}_{\mathrm{II}}\right] \lambda 3727$ ratio, the more likely it is to lead to the lower branch of R23 results (with much lower metallicities).

We finally adopt as values for metallicity those estimated from the 3rd epoch, which has the least afterglow contamination. The mean values after the base metallicity conversion are $12+\log (\mathrm{O} / \mathrm{H})=8.53_{-0.13}^{+0.14}, 8.63 \pm 0.05$ and $8.55 \pm$ 0.02 , for the $1 \mathrm{st}$ and the 2 nd component and for the entire galaxy (combined flux), respectively. Hence, despite the part of the galaxy hosting the GRB having a slightly lower metallicity, the metallicities of both parts are consistent within errors.

In Fig. 12 we plot the position of the two SF regions in a BPT diagram, together with other long and short GRB hosts and GRB sites, colour-coded by redshift. There is no obvious trend with redshift among long GRB hosts in their location in the BPT diagram, indicating that the ionisation conditions in GRB hosts might not be redshift-dependent. The two parts of the host galaxy are located well among other long GRB hosts and sites within the SF branch of the BPT diagram, but not in a very extreme region.

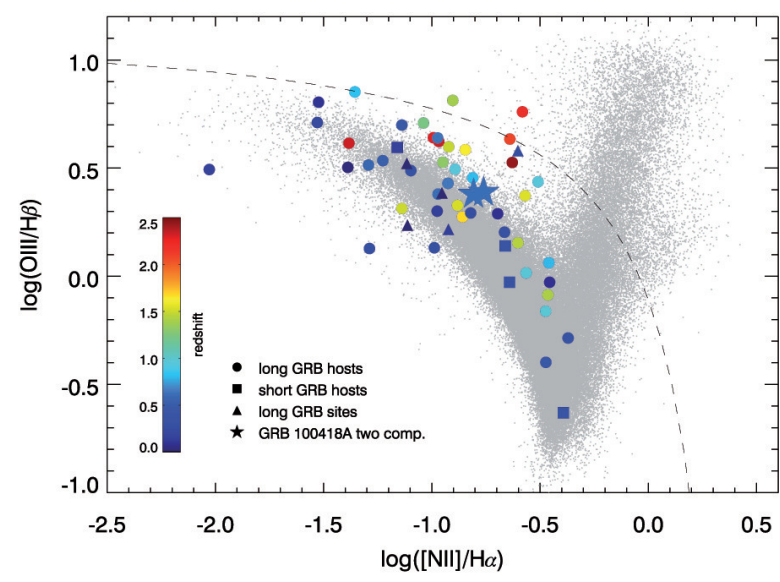

Fig. 12. BPT diagram for long and short GRB hosts and GRB sites from the literature compared to SDSS galaxies (grey dots) and the two SF regions in the host of GRB 100418A (stars). The redshift of the hosts is colour-coded in the plotted symbols. Emission line fluxes used are taken from Christensen et al. (2008), Berger (2009), Han et al. (2010), Levesque et al. (2010), Perley et al. (2012), Schulze et al. (2014), Thöne et al. (2014), Krühler et al. (2015), Stanway et al. (2015), Vergani et al. (2017), Izzo et al. (2017).

As mentioned above, the line ratios clearly indicate excitation by massive stars and not by an AGN. Both parts of the host are at a very similar location, as is their metallicity and other properties, which means that they are two SF regions in the same galaxy and not an interacting system that would usually show larger differences.

In Fig. 13 we plot the mass-metallicity relation for GRB hosts, including the two SF regions of the host. In contrast to many other long GRB hosts, which usually fall below the massmetallicity relation, the reason for which has been extensively discussed in many papers (see e.g. Vergani et al. 2017), the host of GRB 100418A is fully consistent with the low-redshift massmetallicity relation for SDSS galaxies. This is surprising considering that low metallicity - at least at the GRB site - seems to be a common trait among GRB hosts. GRB progenitor models require them to have high angular momentum to produce an accretion disk and launching a jet upon core collapse. However, they need to have retained their fast rotation throughout a phase where they lost their outer layers, which had to have happened given the fact that their accompanying $\mathrm{SNe}$ are of Type Ic. The metallicity of the host of 100418A is almost solar and only a few other hosts have had such relatively high metallicities (see Fig. 13). Why the host was still able to produce a GRB progenitor we can only speculate about, e.g. the star could have been stripped of its outer layers by a binary companion, a model that has gained popularity in the last years given that most massive stars are in binary systems (Sana et al. 2012; Langer 2012).

\subsection{Host-galaxy kinematics}

The resolution of $\mathrm{X}$-shooter and the simultaneous detection of emission and absorption lines in a single spectrum allows us to have a closer look at the galaxy kinematics. The structure in the emission lines with two main knots is visible in all three epochs but with slightly different relative strength and spatial extent/orientation due to the different orientation of the slit (see also Fig. 11). In imaging we do not see any hint of a double component, only a small elongation in $\mathrm{N}-\mathrm{S}$ direction, hence the two regions are aligned close to our line-of-sight. As expected, the 


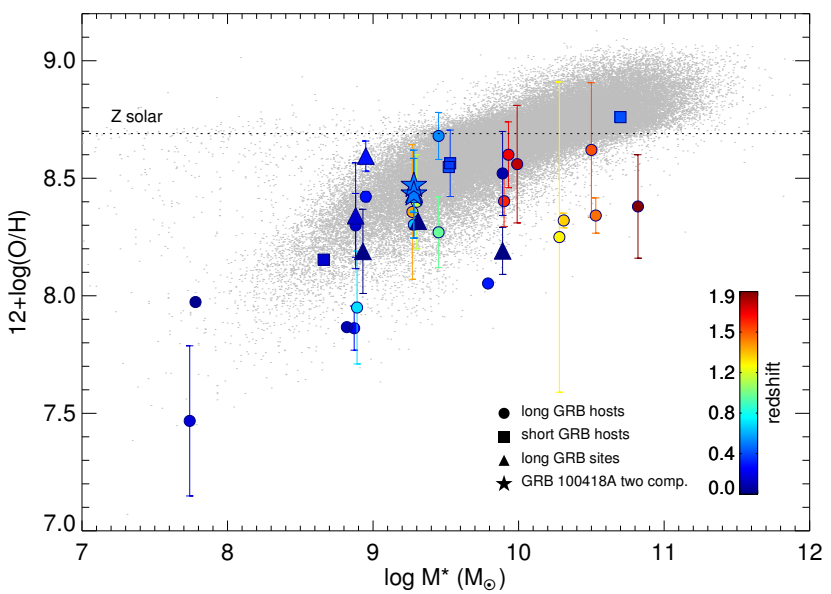

Fig. 13. Mass-metallicity relation for GRB hosts and the two SF regions in the host of 100418A. Grey dots are galaxies from the SDSS DR 10. References are the same as in Fig. 12 with some additional metallicities not derived via the N2 calibrator taken from Vergani et al. (2017) and Krühler et al. (2015).

components in epoch 2 and 3 are very similar to each other since their slit orientation is very similar and they are also the two slits most aligned with the orientation of the galaxy. Epoch 1 differs somewhat due to an orientation almost perpendicular to the orientation of the galaxy. As we concluded above, the very similar abundances in both knots implies that the two main components are likely just two large SF regions in the host and not a pair of dwarf galaxies in close interaction.

We fit several Gaussian components to the strongest emission lines of $\mathrm{H} \beta$ and $[\mathrm{O} \mathrm{III}]$ as proxies for neutral and ionised gas. $\mathrm{H} \alpha$ is in the NIR arm of X-shooter and has a lower resolution and $\mathrm{S} / \mathrm{N}$, hence we use $\mathrm{H} \beta$ instead. In the following we name the two main emission components "blue" and "red blob" according to their relative line-of-sight velocities. The red blob is likely the SF region hosting the actual GRB and has stronger emission lines. In Fig. 14 we plot the fit to the $\mathrm{H} \beta$ and [O $\mathrm{III}]$ lines which we describe below as well as the two lines together with $\mathrm{He}_{\mathrm{I}}$ and $[\mathrm{Ne} \mathrm{III}]$ emission to compare their relative kinematics. We do not perform component fits for the latter two due to their much lower $\mathrm{S} / \mathrm{N}$.

The blue blob is largely dominated by one single emission component, while the red blob can be divided in three narrow components with FWHM between 30 and $40 \mathrm{~km} \mathrm{~s}^{-1}$. In the red wing of the red blob, an additional broad component with a $\sigma$ of $80 \mathrm{~km} \mathrm{~s}^{-1}$ is needed while the situation is less clear in the blue wing, at least for $\mathrm{H} \beta$ which could be affected by stellar absorption as can be seen in the spectra, however, the $\mathrm{S} / \mathrm{N}$ is too low and the spectra go below zero, making an analysis of this possible absorption complicated. [O III], however, does show an equivalent broad component in the blue wing of the blue blob and possibly even some emission beyond that. Broad components, interpreted as indications for outflows, have been found for the highly SF "Green Pea" galaxies (Amorín et al. 2012), albeit with higher FWHM. We also need an additional component in the "bridge" between the two blobs, which we fit with a single broader component, probably a blend of several narrow components, but our resolution does not allow us to disentangle those. The components of epoch $2+3$ in both $\mathrm{H} \beta$ and [O III] are slightly shifted to the red, especially in the blue blob and components change relative strength in the red blob. Epoch 1 is hence probably probing some blue shifted gas in the blue blob which is outside the main N-S orientation of the host.
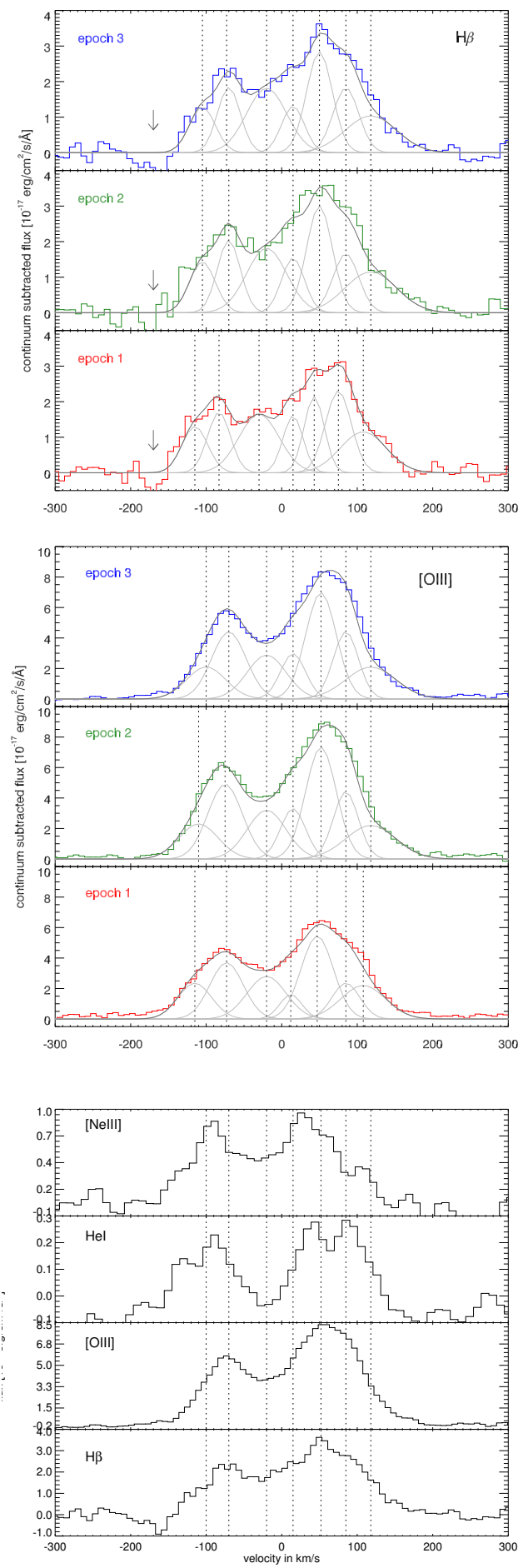

Fig. 14. Gaussian fits to the $\mathrm{H} \beta$ (top panel) and [O III] (middle panel) emission lines at the three epochs of X-shooter spectra analysed here. The arrows in the plot of $\mathrm{H} \beta$ indicate a possible stellar absorption component that we do not fit since the flux goes to negative values. The bottom panel shows [ $\mathrm{Ne}$ III] and [He I] emission lines from the third epoch compared to $\mathrm{H} \beta /\left[\mathrm{O}_{\mathrm{III}}\right]$ and the main (narrow) components fitted in $\mathrm{H} \beta$ indicated for comparison.

Another surprising observation can be done comparing [Ne III] and $\mathrm{He}_{\mathrm{I}}$ with $\mathrm{H} \beta$ and [O III], shown in Fig. 14. For both [Ne III] and $\mathrm{He}$ I, the blue blob is clearly blue shifted compared to the blue blob in $\left[\mathrm{O}\right.$ III] and $\mathrm{H} \beta$. For $\mathrm{He}_{\mathrm{I}}$, the components of the red blob match more or less to the ones of [O III] and $\mathrm{H} \beta$, however, the relative strength is different, with the main emission 
component of [O III] and $\mathrm{H} \beta$ being weaker in He I. For [Ne III], the main component in the red blob is clearly blueshifted compared to $\mathrm{H} \beta$. As mentioned in Sect. 5.3, [Ne III] and [O III] are isoelectric equivalents, have similar ionisation energies, and are both produced in the $\alpha$ process in massive stars, hence they are usually expected and have been observed to be co-spatial and their ratio is constant with metallicity (Izotov et al. 2011). One possibility to change their observed strength is extinction, which affects [O III] more than [Ne III], however, $\mathrm{H} \alpha$ shows the same shape as $\mathrm{H} \beta$ in our spectra, ruling out this possibility. Another way is an enhanced production of the $\mathrm{Ne}^{22}$ isotope in e.g. WR stars, which has been observed in $\mathrm{z} \sim 2$ galaxies (Zeimann et al. $2015)$. However, the flux ratio of $[\mathrm{Ne}$ III $] /\left[\mathrm{O}_{\mathrm{III}}\right]$ is consistent with the normal ratio observed in SDSS galaxies, only the position of the line is different, rather pointing to a difference in e.g. ionisation processes or differential outflows than a large difference in the element production. He $\mathrm{I}$ is only found in very young populations but dependent on temperature and density, hence the different relative strength compared to $\mathrm{H} \beta$ might simply be a reflection of different conditions throughout the host.

For the host of GRB 100418A we can furthermore compare absorption components from cold gas in the GRB sightline with the emitting gas from the galaxy. As mentioned in Sect. 5.2, the absorbing gas probably has a different composition in the different components. Also, absorption and emission components mostly do not trace each other: only the two components with the highest redshift are consistent with the component of the "bridge" and one of the components in the red blob. The other absorption components span a much larger velocity range to the blue of the host complex with a total span of $>500 \mathrm{~km} \mathrm{~s}^{-1}$. The largest velocities are reached in Fe II and $\mathrm{Mg}$ I and $\mathrm{Mg}$ II while the equally strong Ca II only shows absorption within the velocity range of the host emission (see Fig. 11). Mg II is often detected in extended halos of galaxies at large impact factors (see e.g. Churchill et al. 2005; Werk et al. 2012) hence the absorption could correspond to cold gas around the galaxy complex or be indicative of outflows from the SF region, in addition to possibly observed outflows in the emitting gas (see above). A similar difference in velocity span between absorption and emission had been detected for the host of GRB 030329 (Thöne et al. 2007) and this was interpreted as a starburst wind driving metal rich gas out of the very low mass host.

\section{Conclusions}

In this paper we present a complete data set on GRB 100418A and its host galaxy, at a redshift of $z=0.6239$. Observations include data ranging from radio to X-rays, with particularly high quality VLT/X-shooter spectroscopy, obtained at three epochs of the afterglow evolution. These spectra show a large number of both absorption and emission features that allow us to perform an extensive analysis of the host galaxy of this event.

The afterglow is characterised by a very rapid rebrightening in the optical, approximately $2.4 \mathrm{~h}$ after the burst onset, with a brightness increase of $3.4 \pm 0.5 \mathrm{mag}$. Similar very steep rebrightenings have been previously observed in other GRB afterglows during the first day after the burst. While the overall broadband evolution of the afterglow can be satisfactorily explained within the standard fireball model, the spectral slope of $\alpha_{\text {rise }}=5.4 \pm 1.1$ during the rebrightening rise cannot, and requires another interpretation. Although a complete, satisfactory explanation for these steep light curve rebrightenings is still lacking our observations favour an intense and delayed central engine activity.

Late analysis of the light curve indicates the presence of a faint supernova with an absolute magnitude of $M_{V}=-18.49 \pm$
$0.13 \mathrm{mag}$ at the time when one would expect the supernova to reach its maximum. The bright host galaxy, which is over 2.5 magnitudes brighter than the supernova at its peak time complicates this analysis. The SN associated with GRB 100418A is among the faintest GRB-supernova detected to date. We caution that the limited available data at the time of the supernova does not allow us to make a full analysis of its evolution and characteristics. Such a faint supernova is indicative of the varied characteristics of events of this type.

The host of GRB 100418A is a prototypical GRB host at $z \sim 0.6$, but with higher SFR and SSFR and a metallicity $(12+\log (\mathrm{O} / \mathrm{H})=8.55)$ consistent with the local mass-metallicity relation. While it appears very compact in imaging, the spectral resolution of $\mathrm{X}$-shooter and the detection of a large number of bright emission lines allow us to determine both its emissionand absorption-line kinematics. The compact galaxy resolves into two distinct knots with similar properties suggesting that they are two large SF regions of a dwarf galaxy and not a system in a late stage of merging. The absorption lines span a velocity range of $>500 \mathrm{~km} \mathrm{~s}^{-1}$, while the hot emitting gas only reaches a total velocity of $\sim 300 \mathrm{~km} \mathrm{~s}^{-1}$. Both broad wings in the emission lines and the high velocities of the absorption lines point to outflows of metal-enriched gas from the intense star formation in the host, similar to what has been observed for green pea galaxies and some GRB hosts.

Acknowledgements. AdUP, CCT, KB, DAK, LI, and ZC acknowledge support from the Spanish Ministry of Economy and Competitivity under grant number AYA 2014-58381-P; in addition, AdUP and CCT from Ramón y Cajal fellowships (RyC-2012-09975 and RyC-2012-09984). DAK also acknowledges financial support from Juan de la Cierva Incorporación fellowship IJCI-2015-26153. RF acknowledges support from European Regional Development Fund-Project "Engineering applications of microworld physics" (No. CZ.02.1.01/0.0/0.0/16_019/0000766). IB, IKh, RB, and SM acknowledge TUBITAK, IKI, KFU, and AST for partial support in using RTT150. This work was partially funded by the subsidy 3.6714.2017/8.9 allocated to Kazan Federal University for the state assignment in the sphere of scientific activities. TK acknowledges support from the DFG cluster of excellence "Origin and Structure of the Universe". PS acknowledges support through the Sofja Kovalevskaja Award from the Alexander von Humboldt Foundation of Germany. RSR acknwoledges support from ASI (Italian Space Agency) through Contract n. 2015046-R.0 and from European Union Horizon 2020 Programme under the AHEAD project (grant agreement n. 654215). The Cosmic Dawn Center is funded by the DNRF. Based on observations made with the Gran Telescopio Canarias (GTC), installed in the Spanish Observatorio del Roque de los Muchachos of the Instituto de Astrofísica de Canarias, in the island of La Palma. The Submillimeter Array is a joint project between the Smithsonian Astrophysical Observatory and the Academia Sinica Institute of Astronomy and Astrophysics and is funded by the Smithsonian Institution and the Academia Sinica. This work is based on observations carried out under project number U051 with the IRAM Plateau de Bure Interferometer. IRAM is supported by INSU/CNRS (France), MPG (Germany), and IGN (Spain). Some of the data presented herein were obtained at the W. M. Keck Observatory, which is operated as a scientific partnership among the California Institute of Technology, the University of California, and the National Aeronautics and Space Administration. The Observatory was made possible by the generous financial support of the W. M. Keck Foundation. This work is based on observations made with the Spitzer Space Telescope, which is operated by the Jet Propulsion Laboratory, California Institute of Technology under a contract with NASA. Support for this work was provided by NASA through an award issued by JPL/Caltech. The UKIRT data were pipeline processed at the Cambridge Astronomical Survey Unit, and are archived at the Wide Field Astronomy Unit at the Royal Observatory Edinburgh This work made use of data supplied by the UK Swift Science Data Centre at the University of Leicester.

\section{References}

Alard, C. 2000, A\&AS, 144, 363

Alard, C., \& Lupton, R. H. 1998, ApJ, 503, 325

Aller, L. H. 1984, in Physics of Thermal Gaseous Nebulae, ed. L. H. Aller, Astrophys. Space Sci. Lib., 112

Amorín, R., Vílchez, J. M., Hägele, G. F., et al. 2012, ApJ, 754, L22 
Antonelli, L. A., Maund, J. R., Palazzi, E., et al. 2010, GRB Coordinates Network, 10620,

Arnouts, S., Cristiani, S., Moscardini, L., et al. 1999, MNRAS, 310, 540

Becerra, R. L., Watson, A. M., Lee, W. H., et al. 2017, ApJ, 837, 116

Benjamin, R. A., Skillman, E. D., \& Smits, D. P. 1999, ApJ, 514, 307

Berger, E. 2009, ApJ, 690, 231

Berger, E., Kulkarni, S. R., Pooley, G., et al. 2003, Nature, 426, 154

Bianco, F. B., Modjaz, M., Oh, S. M., et al. 2016, Astron. Comput., 16, 54

Bikmaev, I., Khamitov, I., Melnikov, S., et al. 2010, GRB Coordinates Network, 10635

Bohlin, R. C., Colina, L., \& Finley, D. S. 1995, AJ, 110, 1316

Bruzual, G., \& Charlot, S. 2003, MNRAS, 344, 1000

Calzetti, D., Armus, L., Bohlin, R. C., et al. 2000, ApJ, 533, 682

Cano, Z. 2014, ApJ, 794, 121

Cano, Z., Bersier, D., Guidorzi, C., et al. 2011, ApJ, 740, 41

Cano, Z., de Ugarte Postigo, A., Pozanenko, A., et al. 2014, A\&A, 568, A19

Cano, Z., de Ugarte Postigo, A., Perley, D., et al. 2015, MNRAS, 452, 1535

Cano, Z., Izzo, L., de Ugarte Postigo, A., et al. 2017a, A\&A, 605, A107

Cano, Z., Wang, S.-Q., Dai, Z.-G., \& Wu, X.-F. 2017b, Adv. Astron., 2017, 8929054

Chandra, P., \& Frail, D. A. 2012, ApJ, 746, 156

Christensen, L., Vreeswijk, P. M., Sollerman, J., et al. 2008, A\&A, 490, 45

Churchill, C. W., Kacprzak, G. G., \& Steidel, C. C. 2005, in IAU Colloq. 199 Probing Galaxies through Quasar Absorption Lines, eds. P. Williams, C. G. Shu, \& B. Menard, 24

Cucchiara, A., \& Fox, D. B. 2010, GRB Coordinates Network, 10624

Curran, P. A., Evans, P. A., de Pasquale, M., Page, M. J., \& van der Horst, A. J. 2010, ApJ, 716, L135

Dai, Z. G., \& Lu, T. 1998, A\&A, 333, L87

D’Elia, V., Fiore, F., Perna, R., et al. 2009, ApJ, 694, 332

Della Valle, M., Chincarini, G., Panagia, N., et al. 2006, Nature, 444, 1050

Denicoló, G., Terlevich, R., \& Terlevich, E. 2002, MNRAS, 330, 69

de Ugarte Postigo, A., Castro-Tirado, A. J., Gorosabel, J., et al. 2005, A\&A, 443, 841

de Ugarte Postigo, A., Thöne, C. C., Goldoni, P., Fynbo, J. P. U., \& X-shooter GRB Collaboration 2011, Astron. Nachr., 332, 297

de Ugarte Postigo, A., Lundgren, A., Martín, S., et al. 2012a, A\&A, 538, A44

de Ugarte Postigo, A., Fynbo, J. P. U., Thöne, C. C., et al. 2012b, A\&A, 548, A11

de Ugarte Postigo, A., Blazek, M., Janout, P., et al. 2014, in Software and Cyberinfrastructure for Astronomy III, Proc. SPIE, 9152, 91520B

de Ugarte Postigo, A., Schulze, S., Bremer, M., et al. 2017, GRB Coordinates Network, 22187

Diaz, A. I., Pagel, B. E. J., \& Terlevich, E. 1985, MNRAS, 214, 41P

Evans, P. A., Beardmore, A. P., Page, K. L., et al. 2007, A\&A, 469, 379

Evans, P. A., Beardmore, A. P., Page, K. L., et al. 2009, MNRAS, 397, 1177

Filgas, R., Klose, S., \& Greiner, J. 2010, GRB Coordinates Network, 10617

Filgas, R., Krühler, T., Greiner, J., et al. 2011, A\&A, 526, A113

Fontana, A., \& Ballester, P. 1995, The Messenger, 80, 37

Fynbo, J. P. U., Watson, D., Thöne, C. C., et al. 2006, Nature, 444, 1047

Galama, T. J., Vreeswijk, P. M., van Paradijs, J., et al. 1998, Nature, 395, 670

Gallagher, J. S., Hunter, D. A., \& Bushouse, H. 1989, AJ, 97, 700

Gao, H., Lei, W.-H., Zou, Y.-C., Wu, X.-F., \& Zhang, B. 2013, New Astron. Rev., 57, 141

Goldoni, P., Royer, F., François, P., et al. 2006, Proc. SPIE, 6269, 62692K

González Delgado, R. M. 1999, ApJS, 125, 479

Goodman, J. 1997, New Astron., 2, 449

Greiner, J., Krühler, T., Nardini, M., et al. 2013, A\&A, 560, A70

Guseva, N. G., Izotov, Y. I., Fricke, K. J., \& Henkel, C. 2011, A\&A, 534, A84

Han, X. H., Hammer, F., Liang, Y. C., et al. 2010, A\&A, 514, A24

Hjorth, J., Sollerman, J., Møller, P., et al. 2003, Nature, 423, 847

Hjorth, J., Malesani, D., Jakobsson, P., et al. 2012, ApJ, 756, 187

Horváth, I., Bagoly, Z., Balázs, L. G., et al. 2010, ApJ, 713, 552

Ilbert, O., Arnouts, S., McCracken, H. J., et al. 2006, A\&A, 457, 841

Imatani, R., Tomida, H., Nakahira, S., et al. 2016, PASJ, 68, S29

Izotov, Y. I., Stasińska, G., Meynet, G., Guseva, N. G., \& Thuan, T. X. 2006 A\&A, 448, 955

Izotov, Y. I., Guseva, N. G., \& Thuan, T. X. 2011, ApJ, 728, 161

Izzo, L., Thöne, C. C., Schulze, S., et al. 2017, MNRAS, 472, 4480

Jakobsson, P., Hjorth, J., Ramirez-Ruiz, E., et al. 2004, New Astron., 9, 435

Japelj, J., Covino, S., Gomboc, A., et al. 2015, A\&A, 579, A74

Japelj, J., Vergani, S. D., Salvaterra, R., et al. 2016, A\&A, 590, A129

Jelínek, M., Castro-Tirado, A. J., Cunniffe, R., et al. 2016, Adv. Astron., 2016, 192846

Jester, S., Schneider, D. P., Richards, G. T., et al. 2005, AJ, 130, 873

Kann, D. A., Klose, S., \& Zeh, A. 2006, ApJ, 641, 993

Kann, D. A., Klose, S., Zhang, B., et al. 2010, ApJ, 720, 1513

Kann, D. A., Schady, P., Olivares, F. E., et al. 2016, A\&A, submitted
[arXiv:1606.06791]

Kann, D. A., Schady, P., Olivares, E., F., , et al. 2018, A\&A, 617, A122

Kehrig, C., Vílchez, J. M., Telles, E., Cuisinier, F., \& Pérez-Montero, E. 2006, A\&A, 457, 477

Kehrig, C., Vílchez, J. M., Pérez-Montero, E., et al. 2015, ApJ, 801, L28

Kennicutt, Jr., R. C. 1992, ApJ, 388, 310

Kennicutt, Jr., R. C., Tamblyn, P., \& Congdon, C. E. 1994, ApJ, 435, 22

Kewley, L. J., \& Dopita, M. A. 2002, ApJS, 142, 35

Kewley, L. J., \& Ellison, S. L. 2008, ApJ, 681, 1183

Klein, C. R., Morgan, A. N., Perley, D. A., \& Bloom, J. S. 2010, GRB Coordinates Network, 10627

Kobulnicky, H. A., \& Kewley, L. J. 2004, ApJ, 617, 240

Kouveliotou, C., Meegan, C. A., Fishman, G. J., et al. 1993, ApJ, 413, L101

Krühler, T., Malesani, D., Fynbo, J. P. U., et al. 2015, A\&A, 581, A125

Langer, N. 2012, ARA\&A, 50, 107

Laskar, T., Berger, E., Margutti, R., et al. 2015, ApJ, 814, 1

Leitherer, C., Ferguson, H. C., Heckman, T. M., \& Lowenthal, J. D. 1995, ApJ, 454, L19

Levesque, E. M., Kewley, L. J., Berger, E., \& Zahid, H. J. 2010, AJ, 140, 1557

Li, X., \& Hjorth, J. 2014, ArXiv e-prints [arXiv:1407. 3506]

Lü, H.-J., Lan, L., Zhang, B., et al. 2018, ApJ, 862, 130

Maiolino, R., Nagao, T., Grazian, A., et al. 2008, A\&A, 488, 463

Margutti, R., Chincarini, G., Covino, S., et al. 2007, A\&A, 474, 815

Marino, R. A., Rosales-Ortega, F. F., Sánchez, S. F., et al. 2013, A\&A, 559, A114

Marshall, F. E., Beardmore, A. P., Gelbord, J. M., et al. 2010, GRB Coordinates Network, 10612

Marshall, F. E., Antonelli, L. A., Burrows, D. N., et al. 2011, ApJ, 727, 132

Martin, S., Petitpas, G., de Ugarte Postigo, A., et al. 2010, GRB Coordinates Network, 10630

Massey, P. 1998, ApJ, 501, 153

McGaugh, S. S. 1991, ApJ, 380, 140

Melandri, A., Pian, E., D'Elia, V., et al. 2014, A\&A, 567, A29

Mészáros, P., \& Rees, M. J. 1999, MNRAS, 306, L39

Mészáros, P., Rees, M. J., \& Wijers, R. A. M. J. 1998, ApJ, 499, 301

Michałowski, M. J., Xu, D., Stevens, J., et al. 2018, A\&A, 616, A169

Modigliani, A., Goldoni, P., Royer, F., et al. 2010, Proc. SPIE, 7737, 773728

Moin, A., Chandra, P., Miller-Jones, J. C. A., et al. 2013, ApJ, 779, 105

Nakar, E., \& Oren, Y. 2004, ApJ, 602, L97

Nakar, E., \& Piran, T. 2004, MNRAS, 353, 647

Nardini, M., Greiner, J., Krühler, T., et al. 2011, A\&A, 531, A39

Niino, Y., Hashimoto, T., Aoki, K., et al. 2012, PASJ, 64, 115

Olivares, E., F., Greiner, J., Schady, P., et al. 2015, A\&A, 577, A44

Osterbrock, D. E., \& Ferland, G. J. 2006, Astrophysics of Gaseous Nebulae and Active Galactic Nuclei (Sausalito, CA: University Science Books)

Paczyński, B. 1998, ApJ, 494, L45

Pagani, C., Beardmore, A. P., \& Marshall, F. E. 2010, GRB Coordinates Network, 10622

Pandey, S. B., Zheng, W., Xiao, L., \& Guver, T. 2010, GRB Coordinates Network, 10613

Pearson, R., Moody, J. W., \& Pace, C. 2010, GRB Coordinates Network, 10626 Pérez-Montero, E., Díaz, A. I., Vílchez, J. M., \& Kehrig, C. 2006, A\&A, 449, 193

Perley, D. A., Modjaz, M., Morgan, A. N., et al. 2012, ApJ, 758, 122

Perley, D. A., Krühler, T., Schulze, S., et al. 2016a, ApJ, 817, 7

Perley, D. A., Tanvir, N. R., Hjorth, J., et al. 2016b, ApJ, 817, 8

Pettini, M., \& Pagel, B. E. J. 2004, MNRAS, 348, L59

Pilyugin, L. S., Vílchez, J. M., \& Thuan, T. X. 2010, ApJ, 720, 1738

Piran, T. 1999, Phys. Rep., 314, 575

Planck Collaboration XIII 2016, A\&A, 594, A13

Proxauf, B., Öttl, S., \& Kimeswenger, S. 2014, A\&A, 561, A10

Quimby, R. M., Kulkarni, S. R., Kasliwal, M. M., et al. 2011, Nature, 474, 487

Ramirez-Ruiz, E., Dray, L. M., Madau, P., \& Tout, C. A. 2001, MNRAS, 327, 829

Rees, M. J., \& Mészáros, P. 1998, ApJ, 496, L1

Resmi, L., Ishwara-Chandra, C. H., Castro-Tirado, A. J., et al. 2005, A\&A, 440, 477

Rhoads, J. E. 1997, ApJ, 487, L1

Richardson, D. 2009, AJ, 137, 347

Salvaterra, R., Della Valle, M., Campana, S., et al. 2009, Nature, 461, 1258

Sana, H., de Mink, S. E., de Koter, A., et al. 2012, Science, 337, 444

Sari, R., \& Mészáros, P. 2000, ApJ, 535, L33

Sari, R., Piran, T., \& Narayan, R. 1998, ApJ, 497, L17

Sault, R. J., Teuben, P. J., \& Wright, M. C. H. 1995, in Astronomical Data Analysis Software and Systems IV, eds. R. A. Shaw, H. E. Payne, \& J. J. E. Hayes, ASP Conf. Ser., 77, 433

Schady, P., Dwelly, T., Page, M. J., et al. 2012, A\&A, 537, A15

Schaerer, D. 2003, A\&A, 397, 527

Schlafly, E. F., \& Finkbeiner, D. P. 2011, ApJ, 737, 103 
Schulze, S., Malesani, D., Cucchiara, A., et al. 2014, A\&A, 566, A102

Selsing, J., Malesani, D., Goldoni, P., et al. 2018, A\&A, submitted [arXiv:1802.07727]

Sheth, K., Frail, D. A., White, S., et al. 2003, ApJ, 595, L33

Stanway, E. R., Levan, A. J., Tanvir, N., et al. 2015, MNRAS, 446, 3911

Starling, R. L. C., Wijers, R. A. M. J., Wiersema, K., et al. 2007, ApJ, 661, 787

Starling, R. L. C., van der Horst, A. J., Rol, E., et al. 2008, ApJ, 672, 433

Strolger, L.-G., Riess, A. G., Dahlen, T., et al. 2004, ApJ, 613, 200

Tanga, M., Krühler, T., Schady, P., et al. 2018, A\&A, 615, A136

Tanvir, N. R., Fox, D. B., Levan, A. J., et al. 2009, Nature, 461, 1254

Tanvir, N. R., Fynbo, J. P. U., de Ugarte Postigo, A., et al. 2018, ArXiv e-prints [arXiv:1805.07318]

Thöne, C. C., Greiner, J., Savaglio, S., \& Jehin, E. 2007, ApJ, 671, 628

Thöne, C. C., Christensen, L., Prochaska, J. X., et al. 2014, MNRAS, 441, 2034

Tody, D. 1993, in Astronomical Data Analysis Software and Systems II, eds. R.

J. Hanisch, R. J. V. Brissenden, \& J. Barnes, ASP Conf. Ser., 52, 173

Ukwatta, T. N., Barthelmy, S. D., Baumgartner, W. H., et al. 2010, GRB Coordinates Network, 10615

Updike, A. C., Hartmann, D. H., \& Murphy, B. 2010, GRB Coordinates Network, 10619

van Eerten, H. 2014, MNRAS, 442, 3495

Vergani, S. D., Salvaterra, R., Japelj, J., et al. 2015, A\&A, 581, A102

Vergani, S. D., Palmerio, J., Salvaterra, R., et al. 2017, A\&A, 599, A120

Vernet, J., Dekker, H., D'Odorico, S., et al. 2011, A\&A, 536, A105

Vreeswijk, P. M., Ledoux, C., Smette, A., et al. 2007, A\&A, 468, 83

Vreeswijk, P. M., Ledoux, C., Raassen, A. J. J., et al. 2013, A\&A, 549, A22

Wang, X., \& Loeb, A. 2000, ApJ, 535, 788

Werk, J. K., Prochaska, J. X., Thom, C., et al. 2012, ApJS, 198, 3

Wiersema, K., Savaglio, S., Vreeswijk, P. M., et al. 2007, A\&A, 464, 529

$\mathrm{Xu}, \mathrm{D}$., de Ugarte Postigo, A., Leloudas, G., et al. 2013, ApJ, 776, 98

Zafar, T., Watson, D., Fynbo, J. P. U., et al. 2011, A\&A, 532, A143

Zafar, T., Watson, D., Møller, P., et al. 2018, MNRAS, 479, 1542

Zaritsky, D., Kennicutt, Jr., R. C., \& Huchra, J. P. 1994, ApJ, 420, 87

Zeh, A., Klose, S., \& Hartmann, D. H. 2004, ApJ, 609, 952

Zeimann, G. R., Ciardullo, R., Gebhardt, H., et al. 2015, ApJ, 798, 29

Zhang, B., \& Mészáros, P. 2002, ApJ, 566, 712

Zhang, B., Fan, Y. Z., Dyks, J., et al. 2006, ApJ, 642, 354

1 Instituto de Astrofísica de Andalucía, Glorieta de la Astronomía s/n, 18008 Granada e-mail: deugarte@iaa.es

2 Dark Cosmology Centre, Niels Bohr Institute, Juliane Maries Vej 30, Copenhagen Ø 2100, Denmark

3 The George Washington University, 725 21st street, NW Washington DC 20052, USA

4 GWU/Astronomy, Physics and Statistics Institute of Sciences (APSIS), USA

5 Astroparticules et Cosmologie, Université Paris Diderot, CNRS/IN2P3, CEA/Irfu, Observatoire de Paris, Sorbonne Paris Cité, 10 rue Alice Domon et Léonie Duquet, 75205 Paris Cedex 13, France

6 Joint ALMA Observatory, Alonso de Córdova 3107, Vitacura 763 0355, Santiago, Chile

7 European Southern Observatory, Alonso de Córdova 3107, Vitacura, Casilla 19001, Santiago 19, Chile
${ }^{8}$ Institute of Experimental and Applied Physics, Czech Technical University in Prague, Horska 3a/22, 12800 Prague, Czech Republic

9 Max-Planck-Institut für Extraterrestrische Physik, Giessenbachstraße, 85748 Garching, Germany

10 Unidad Asociada Grupo Ciencias Planetarias UPV/EHUIAA/CSIC, Departamento de Física Aplicada I, E.T.S. Ingeniería, Universidad del País-Vasco UPV/EHU, Alameda de Urquijo s/n, 48013 Bilbao, Spain

11 Ikerbasque, Basque Foundation for Science, Alameda de Urquijo 36-5, 48008 Bilbao, Spain

12 Kazan Federal University, Kremlevskaya Str., 18, 420008 Kazan, Russia

13 Academy of Sciences of Tatarstan, Bauman Str., 20, 420111 Kazan, Russia

14 Institut de Radioastronomie Millimétrique (IRAM), 300 rue de la Piscine, 38406 Saint Martin d'Héres, France

15 Space Research Institute, Russian Academy of Sciences, Profsoyuznaya 84/32, 117997 Moscow, Russia

16 National Research University Higher School of Economics, Moscow, Russia

17 INAF-Osservatorio Astronomico di Brera, Via E. Bianchi 46, 23807 Merate, LC, Italy

18 Cosmic Dawn Center, Niels Bohr Institute, University of Copenhagen, Juliane Maries Vej 30, 2100 Copenhagen Ø, Denmark

19 Astronomical Institute of the Czech Academy of Sciences, Frikova 298, 25165 Ondrejov, Czech Republic

20 TUBITAK National Observatory, Akdeniz Universitesi Yerleskesi, 07058 Antalya, Turkey

21 Harvard-Smithsonian Center for Astrophysics, 60 Garden Street, Cambridge, MA 02138, USA

22 Universitá degli studi di Milano-Bicocca, Piazza della Scienza 3, 20126 Milano, Italy

23 Astrophysics Research Institute, Liverpool John Moores University, IC2, Liverpool Science Park, 146 Brownlow Hill, Liverpool L3 5RF, UK

24 Harvard-Smithsonian Center for Astrophysics, Submillimeter Array, 645 North A'ohoku Place, Hilo, HI 96720, USA

25 Cavendish Laboratory, JJ Thomson Avenue, Cambridge CB3 OHE, UK

26 Monash Centre for Astrophysics, Monash University, PO Box 27, Clayton, Victoria 3800, Australia

27 School of Physics and Astronomy, Monash University, PO Box 27, Clayton, Victoria 3800, Australia

28 INAF-Istituto Astrofisica e Planetologia Spaziali, Via Fosso Cavaliere 100, 00133 Rome, Italy

29 Department of Physics and Astronomy, University of Leicester, University Road, Leicester LE1 7RH, UK

30 Department of Physics, University of Warwick, Coventry CV4 7AL, UK

31 Anton Pannekoek Institute for Astronomy, University of Amsterdam, PO Box 94249, 1090 GE Amsterdam, The Netherlands

32 Australian Astronomical Observatory, PO Box 915, North Ryde, NSW 1670, Australia 
Appendix A: Additional figure and tables

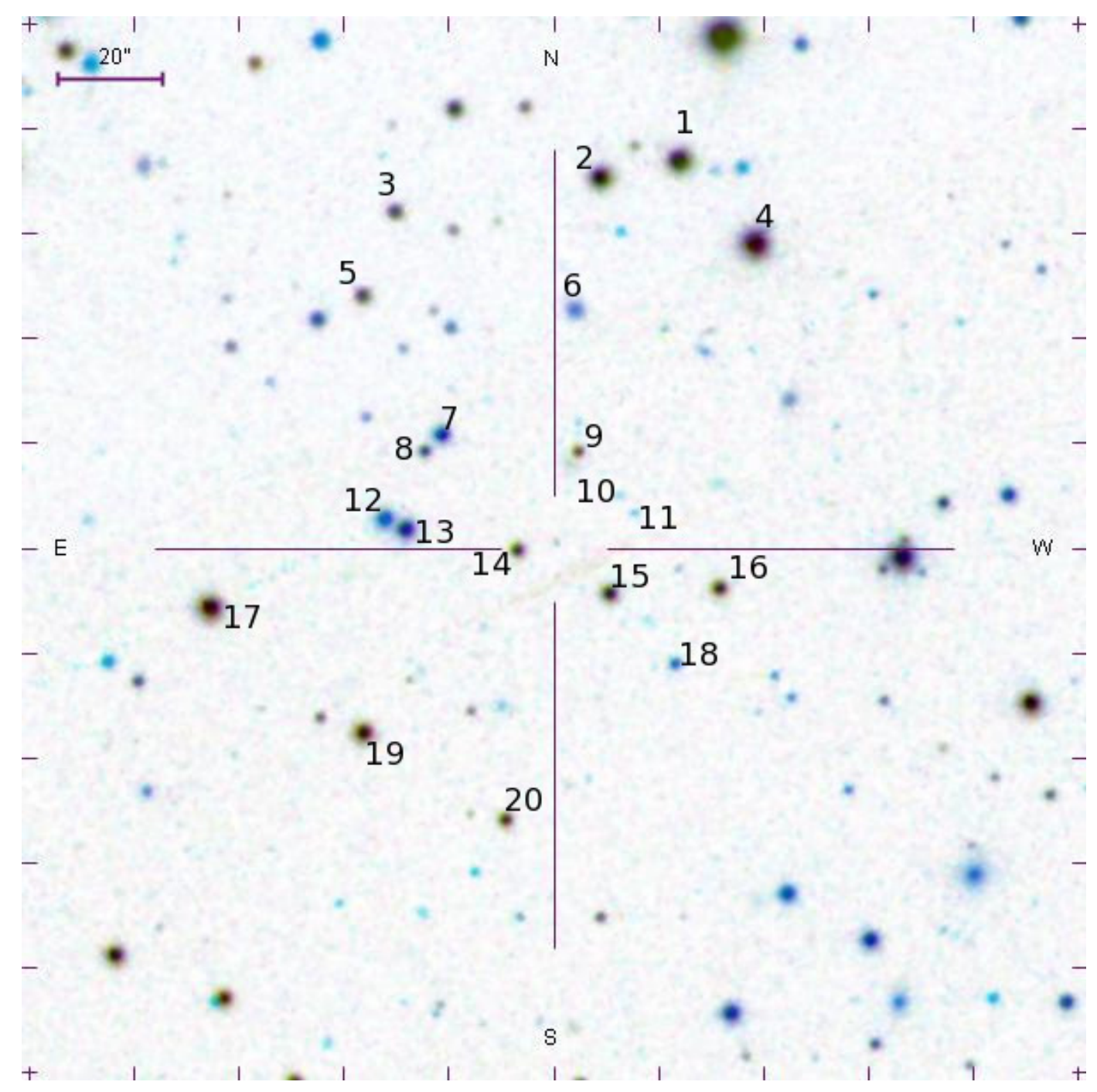

Fig. A.1. Calibration stars used for the optical photometry. 
Table A.1. Optical/NIR observations.

\begin{tabular}{|c|c|c|c|c|}
\hline $\begin{array}{l}T-T_{0} \\
\text { (days) }\end{array}$ & Telescope & Band & Magnitude & $\begin{array}{c}\text { Flux density } \\
(\mu \mathrm{Jy})\end{array}$ \\
\hline 0.07464 & Swift/UVOT & $u v w 2$ & $22.19 \pm 0.25$ & $7.92 \pm 2.06$ \\
\hline 6.24391 & Swift/UVOT & $u v w 2$ & $24.03 \pm 0.30$ & $1.45 \pm 0.47$ \\
\hline 8.33782 & Swift/UVOT & $u v w 2$ & $23.54 \pm 0.22$ & $2.28 \pm 0.52$ \\
\hline 0.01117 & Swift/UVOT & uvm2 & $21.56 \pm 0.34$ & $15.47 \pm 5.71$ \\
\hline 0.06275 & Swift/UVOT & uvm2 & $21.78 \pm 0.22$ & $12.65 \pm 2.89$ \\
\hline 0.07939 & Swift/UVOT & uvm2 & $21.55 \pm 0.20$ & $15.65 \pm 3.20$ \\
\hline 5.25524 & Swift/UVOT & uvm2 & $23.07 \pm 0.19$ & $3.86 \pm 0.73$ \\
\hline 7.89360 & Swift/UVOT & uvm2 & $23.59 \pm 0.23$ & $2.39 \pm 0.56$ \\
\hline 0.01145 & Swift/UVOT & $u v w l$ & $21.42 \pm 0.32$ & $14.90 \pm 5.19$ \\
\hline 0.06513 & Swift/UVOT & $u v w 1$ & $22.01 \pm 0.29$ & $8.61 \pm 2.64$ \\
\hline 0.08176 & Swift/UVOT & $u v w 1$ & $21.77 \pm 0.29$ & $10.72 \pm 3.27$ \\
\hline 2.59007 & Swift/UVOT & $u v w 1$ & $22.25 \pm 0.11$ & $6.89 \pm 0.70$ \\
\hline 6.43383 & Swift/UVOT & $u v w 1$ & $23.52 \pm 0.31$ & $2.14 \pm 0.72$ \\
\hline 8.35208 & Swift/UVOT & $u v w 1$ & $23.68 \pm 0.30$ & $1.85 \pm 0.58$ \\
\hline 0.00187 & Swift/UVOT & white & $21.46 \pm 0.15$ & $12.57 \pm 1.91$ \\
\hline 0.01035 & Swift/UVOT & white & $21.67 \pm 0.17$ & $10.43 \pm 1.73$ \\
\hline 0.07226 & Swift/UVOT & white & $21.14 \pm 0.11$ & $16.98 \pm 1.73$ \\
\hline 0.60178 & Swift/UVOT & white & $20.18 \pm 0.02$ & $40.85 \pm 0.65$ \\
\hline 1.01772 & Swift/UVOT & white & $20.67 \pm 0.02$ & $26.18 \pm 0.58$ \\
\hline 1.76396 & Swift/UVOT & white & $21.30 \pm 0.03$ & $14.60 \pm 0.39$ \\
\hline 2.79714 & Swift/UVOT & white & $21.83 \pm 0.04$ & $8.95 \pm 0.36$ \\
\hline 3.54998 & Swift/UVOT & white & $22.20 \pm 0.04$ & $6.35 \pm 0.22$ \\
\hline 4.43120 & Swift/UVOT & white & $22.26 \pm 0.05$ & $6.03 \pm 0.30$ \\
\hline 5.49919 & Swift/UVOT & white & $22.41 \pm 0.07$ & $5.24 \pm 0.33$ \\
\hline 7.27573 & Swift/UVOT & white & $22.40 \pm 0.10$ & $5.32 \pm 0.50$ \\
\hline 9.32466 & Swift/UVOT & white & $23.01 \pm 0.09$ & $3.01 \pm 0.26$ \\
\hline 10.77969 & Swift/UVOT & white & $23.15 \pm 0.06$ & $2.65 \pm 0.15$ \\
\hline 13.03649 & Swift/UVOT & white & $23.11 \pm 0.05$ & $2.77 \pm 0.14$ \\
\hline 2.3256 & $10.4 \mathrm{mGTC} / \mathrm{OSIRIS}$ & $u^{\prime}$ & $21.55 \pm 0.05$ & $11.58 \pm 0.53$ \\
\hline 4.2138 & $10.4 \mathrm{mGTC} / \mathrm{OSIRIS}$ & $u^{\prime}$ & $21.68 \pm 0.06$ & $10.27 \pm 0.57$ \\
\hline 90.1587 & $10.4 \mathrm{mGTC} / \mathrm{OSIRIS}$ & $u^{\prime}$ & $23.17 \pm 0.06$ & $2.60 \pm 0.14$ \\
\hline 0.07524 & Swift/UVOT & $u$ & $20.98 \pm 0.19$ & $19.86 \pm 3.74$ \\
\hline 5.76062 & Swift/UVOT & $u$ & $22.32 \pm 0.36$ & $5.78 \pm 2.28$ \\
\hline 7.94797 & Swift/UVOT & $u$ & $23.03 \pm 0.23$ & $2.99 \pm 0.72$ \\
\hline 0.06988 & Swift/UVOT & $b$ & $20.27 \pm 0.20$ & $35.81 \pm 7.36$ \\
\hline 6.90466 & Swift/UVOT & $b$ & $22.27 \pm 0.34$ & $5.72 \pm 2.14$ \\
\hline 8.55971 & Swift/UVOT & $b$ & $22.24 \pm 0.36$ & $5.83 \pm 2.26$ \\
\hline 1067.3543 & 10.0mKeck/LRIS & $B$ & $23.23 \pm 0.10$ & $2.63 \pm 0.22$ \\
\hline 1.50975 & 8.2mVLT/X-shooter & $g^{\prime}$ & $20.45 \pm 0.09$ & $29.95 \pm 2.49$ \\
\hline 2.3355 & 10.4mGTC/OSIRIS & $g^{\prime}$ & $21.05 \pm 0.02$ & $17.23 \pm 0.32$ \\
\hline 4.2347 & 10.4mGTC/OSIRIS & $g^{\prime}$ & $21.56 \pm 0.06$ & $10.77 \pm 0.60$ \\
\hline 17.7392 & 10.0mKeck/LRIS & $g^{\prime}$ & $22.71 \pm 0.06$ & $3.74 \pm 0.21$ \\
\hline 28.3172 & 10.4mGTC/OSIRIS & $g^{\prime}$ & $22.94 \pm 0.04$ & $3.02 \pm 0.11$ \\
\hline 90.1744 & 10.4mGTC/OSIRIS & $g^{\prime}$ & $23.01 \pm 0.04$ & $2.83 \pm 0.10$ \\
\hline 2647.101 & 10.4mGTC/OSIRIS & $g^{\prime}$ & $22.99 \pm 0.09$ & $2.87 \pm 0.24$ \\
\hline 6.5145 & 8.2mVLT/FORS2 & V & $21.86 \pm 0.04$ & $7.87 \pm 0.29$ \\
\hline 0.34872 & 8.2mVLT/X-shooter & $r^{\prime}$ & $18.79 \pm 0.04$ & $128.94 \pm 4.75$ \\
\hline 1.44281 & 8.2mVLT/X-shooter & $r^{\prime}$ & $19.85 \pm 0.07$ & $48.57 \pm 3.13$ \\
\hline 1.50698 & $8.2 \mathrm{mVLT} / \mathrm{X}$-shooter & $r^{\prime}$ & $19.98 \pm 0.08$ & $43.09 \pm 3.18$ \\
\hline 2.3394 & 10.4mGTC/OSIRIS & $r^{\prime}$ & $20.59 \pm 0.04$ & $24.57 \pm 0.91$ \\
\hline 2.46029 & 8.2mVLT/X-shooter & $r^{\prime}$ & $20.66 \pm 0.09$ & $23.04 \pm 1.91$ \\
\hline 4.2391 & 10.4mGTC/OSIRIS & $r^{\prime}$ & $21.09 \pm 0.04$ & $15.50 \pm 0.57$ \\
\hline 28.3049 & 10.4mGTC/OSIRIS & $r^{\prime}$ & $22.23 \pm 0.04$ & $5.42 \pm 0.20$ \\
\hline 90.1826 & 10.4mGTC/OSIRIS & $r^{\prime}$ & $22.34 \pm 0.04$ & $4.90 \pm 0.18$ \\
\hline 2646.1457 & 10.4mGTC/OSIRIS & $r^{\prime}$ & $22.43 \pm 0.05$ & $4.51 \pm 0.21$ \\
\hline
\end{tabular}

Notes. Magnitudes are in the $\mathrm{AB}$ system, without correction for galactic extinction, whereas the flux densities have been corrected for it. 
Table A.1. continued.

\begin{tabular}{|c|c|c|c|c|}
\hline $\begin{array}{l}T-T_{0} \\
\text { (days) }\end{array}$ & Telescope & Band & Magnitude & $\begin{array}{c}\text { Flux density } \\
(\mu \mathrm{Jy})\end{array}$ \\
\hline 0.02276 & RTT150 & $R_{C}$ & $20.55 \pm 0.05$ & $25.33 \pm 1.17$ \\
\hline 0.06085 & RTT150 & $R_{C}$ & $21.44 \pm 0.26$ & $11.16 \pm 3.08$ \\
\hline 0.09378 & RTT150 & $R_{C}$ & $19.07 \pm 0.04$ & $99.01 \pm 3.65$ \\
\hline 0.09825 & RTT150 & $R_{C}$ & $18.97 \pm 0.09$ & $108.57 \pm 9.00$ \\
\hline 0.10723 & RTT150 & $R_{C}$ & $18.87 \pm 0.18$ & $119.04 \pm 20.83$ \\
\hline 0.11173 & RTT150 & $R_{C}$ & $18.71 \pm 0.03$ & $137.94 \pm 3.81$ \\
\hline 0.11637 & RTT150 & $R_{C}$ & $18.73 \pm 0.03$ & $135.42 \pm 3.74$ \\
\hline 1.14583 & RTT150 & $R_{C}$ & $19.33 \pm 0.09$ & $77.93 \pm 6.46$ \\
\hline 2.14583 & RTT150 & $R_{C}$ & $20.46 \pm 0.07$ & $27.52 \pm 1.77$ \\
\hline 2.15417 & RTT150 & $R_{C}$ & $20.44 \pm 0.09$ & $28.03 \pm 2.32$ \\
\hline 2.16250 & RTT150 & $R_{C}$ & $20.49 \pm 0.05$ & $26.77 \pm 1.23$ \\
\hline 2.20833 & RTT150 & $R_{C}$ & $20.50 \pm 0.03$ & $26.53 \pm 0.73$ \\
\hline 5.12917 & RTT150 & $R_{C}$ & $20.95 \pm 0.20$ & $17.53 \pm 3.55$ \\
\hline 6.5172 & 8.2mVLT/FORS & $R_{C}$ & $21.52 \pm 0.06$ & $10.36 \pm 0.57$ \\
\hline 9.16667 & RTT150 & $R_{C}$ & $21.66 \pm 0.09$ & $9.11 \pm 0.76$ \\
\hline 13.06250 & RTT150 & $R_{C}$ & $21.98 \pm 0.08$ & $6.79 \pm 0.50$ \\
\hline 14.10417 & RTT150 & $R_{C}$ & $21.98 \pm 0.05$ & $6.79 \pm 0.31$ \\
\hline 15.13750 & RTT150 & $R_{C}$ & $21.96 \pm 0.03$ & $6.91 \pm 0.19$ \\
\hline 16.10417 & RTT150 & $R_{C}$ & $21.95 \pm 0.03$ & $6.98 \pm 0.19$ \\
\hline 17.7395 & 10.0mKeck/LRIS & $R_{C}$ & $22.02 \pm 0.06$ & $6.53 \pm 0.36$ \\
\hline 18.14583 & RTT150 & $R_{C}$ & $22.11 \pm 0.06$ & $6.02 \pm 0.33$ \\
\hline 21.12500 & RTT150 & $R_{C}$ & $22.11 \pm 0.05$ & $6.02 \pm 0.28$ \\
\hline 22.10417 & RTT150 & $R_{C}$ & $22.12 \pm 0.05$ & $5.97 \pm 0.27$ \\
\hline 25.6361 & 8.2mSubaru/FOCAS & $R_{C}$ & $22.31 \pm 0.05$ & $5.00 \pm 0.23$ \\
\hline 57.07083 & RTT150 & $R_{C}$ & $22.24 \pm 0.06$ & $5.34 \pm 0.30$ \\
\hline 106.96389 & RTT 150 & $R_{C}$ & $22.31 \pm 0.05$ & $5.01 \pm 0.23$ \\
\hline 1.51242 & 8.2mVLT/X-shooter & $i^{\prime}$ & $19.63 \pm 0.07$ & $53.65 \pm 3.69$ \\
\hline 2.3419 & 10.4mGTC/OSIRIS & $i^{\prime}$ & $20.22 \pm 0.03$ & $33.24 \pm 0.92$ \\
\hline 4.2435 & 10.4mGTC/OSIRIS & $i^{\prime}$ & $20.66 \pm 0.03$ & $22.16 \pm 0.61$ \\
\hline 28.3131 & 10.4mGTC/OSIRIS & $i^{\prime}$ & $21.86 \pm 0.04$ & $7.34 \pm 0.27$ \\
\hline 90.1961 & 10.4mGTC/OSIRIS & $i^{\prime}$ & $22.07 \pm 0.04$ & $6.04 \pm 0.22$ \\
\hline 2646.1187 & 10.4mGTC/OSIRIS & $i^{\prime}$ & $21.90 \pm 0.06$ & $7.07 \pm 0.39$ \\
\hline 6.5197 & $8.2 \mathrm{mVLT} / \mathrm{FORS}$ & $I_{C}$ & $21.06 \pm 0.03$ & $15.14 \pm 0.42$ \\
\hline 15.3697 & 8.2mVLT/FORS & $I_{C}$ & $21.40 \pm 0.03$ & $11.07 \pm 0.31$ \\
\hline 1067.7231 & 10.0mKeck/LRIS & $I_{C}$ & $21.98 \pm 0.06$ & $6.49 \pm 0.36$ \\
\hline 2.3460 & 10.4mGTC/OSIRIS & $z^{\prime}$ & $20.07 \pm 0.05$ & $37.05 \pm 1.71$ \\
\hline 4.2504 & 10.4mGTC/OSIRIS & $z^{\prime}$ & $20.57 \pm 0.05$ & $23.38 \pm 1.08$ \\
\hline 90.2058 & 10.4mGTC/OSIRIS & $z^{\prime}$ & $22.02 \pm 0.07$ & $6.15 \pm 0.40$ \\
\hline 2647.1253 & 10.4mGTC/OSIRIS & $z^{\prime}$ & $22.03 \pm 0.07$ & $6.09 \pm 0.39$ \\
\hline 1.56641 & 3.8mUKIRT/WFCam & $J$ & $19.07 \pm 0.04$ & $89.70 \pm 3.30$ \\
\hline 1.57644 & 3.8mUKIRT/WFCam & $J$ & $19.03 \pm 0.04$ & $93.07 \pm 3.43$ \\
\hline 5.24920 & $3.5 \mathrm{mCAHA} / \mathrm{O} 2000$ & $J$ & $20.57 \pm 0.08$ & $22.51 \pm 1.66$ \\
\hline 13.15972 & 3.5mCAHA/O2000 & $J$ & $21.81 \pm 0.25$ & $7.18 \pm 1.85$ \\
\hline 41.13148 & $3.5 \mathrm{mCAHA} / \mathrm{O} 2000$ & $J$ & $21.94 \pm 0.13$ & $6.37 \pm 0.82$ \\
\hline 1.56928 & 3.8mUKIRT/WFCam & $H$ & $18.68 \pm 0.03$ & $126.24 \pm 3.49$ \\
\hline 1.57978 & 3.8mUKIRT/WFCam & $H$ & $18.68 \pm 0.03$ & $126.24 \pm 3.49$ \\
\hline 8.11896 & 3.5mCAHA/O2000 & $H$ & $21.01 \pm 0.16$ & $14.82 \pm 2.32$ \\
\hline 1.57269 & 3.8mUKIRT/WFCam & $K_{S}$ & $18.32 \pm 0.02$ & $174.10 \pm 3.21$ \\
\hline 1.58329 & 3.8mUKIRT/WFCam & $K_{S}$ & $18.39 \pm 0.03$ & $163.23 \pm 4.51$ \\
\hline 7.13634 & $3.5 \mathrm{mCAHA} / \mathrm{O} 2000$ & $K_{S}$ & $20.78 \pm 0.20$ & $17.98 \pm 3.64$ \\
\hline 8.15765 & 3.5mCAHA/O2000 & $K_{S}$ & $20.71 \pm 0.19$ & $19.18 \pm 3.71$ \\
\hline 1007.3888 & 4.2mWHT/LIRIS & $K_{S}$ & $21.57 \pm 0.21$ & $8.71 \pm 1.69$ \\
\hline 408.5937 & Spitzer & $3.6 \mu \mathrm{m}$ & $21.98 \pm 0.10$ & $5.86 \pm 0.57$ \\
\hline
\end{tabular}


Table A.2. Calibration stars used for photometry in Sloan bands (AB) and Johnson/Cousins (Vega), as indicated in Fig. A.1.

\begin{tabular}{|c|c|c|c|c|c|}
\hline Star & $u$ & $g$ & $r$ & $i$ & $z$ \\
\hline 1 & $18.40 \pm 0.02$ & $17.07 \pm 0.01$ & $16.53 \pm 0.01$ & $16.33 \pm 0.01$ & $16.24 \pm 0.01$ \\
\hline 2 & $18.94 \pm 0.02$ & $17.47 \pm 0.01$ & $16.93 \pm 0.01$ & $16.73 \pm 0.01$ & $16.62 \pm 0.01$ \\
\hline 3 & $21.20 \pm 0.11$ & $19.48 \pm 0.01$ & $18.82 \pm 0.01$ & $18.56 \pm 0.01$ & $18.42 \pm 0.03$ \\
\hline 4 & $17.35 \pm 0.01$ & $15.82 \pm 0.01$ & $15.27 \pm 0.01$ & $15.09 \pm 0.01$ & $15.00 \pm 0.01$ \\
\hline 5 & $20.99 \pm 0.09$ & $19.36 \pm 0.01$ & $18.74 \pm 0.01$ & $18.49 \pm 0.01$ & $18.31 \pm 0.03$ \\
\hline 6 & - & $20.80 \pm 0.04$ & $19.21 \pm 0.02$ & $18.65 \pm 0.01$ & $18.29 \pm 0.05$ \\
\hline 7 & $21.63 \pm 0.15$ & $19.15 \pm 0.01$ & $17.82 \pm 0.01$ & $17.27 \pm 0.01$ & $16.97 \pm 0.01$ \\
\hline 8 & $22.06 \pm 0.21$ & $20.16 \pm 0.02$ & $19.35 \pm 0.01$ & $18.98 \pm 0.01$ & $18.80 \pm 0.04$ \\
\hline 9 & $21.13 \pm 0.10$ & $19.87 \pm 0.02$ & $19.54 \pm 0.02$ & $19.33 \pm 0.02$ & $19.34 \pm 0.07$ \\
\hline 10 & - & - & $22.69 \pm 0.20$ & $21.60 \pm 0.11$ & - \\
\hline 11 & - & $23.79 \pm 0.33$ & $22.27 \pm 0.13$ & $21.09 \pm 0.07$ & $20.95 \pm 0.28$ \\
\hline 12 & $21.78 \pm 0.17$ & $19.01 \pm 0.01$ & $17.62 \pm 0.01$ & $16.91 \pm 0.01$ & $16.67 \pm 0.01$ \\
\hline 13 & $20.82 \pm 0.08$ & $18.31 \pm 0.01$ & $17.07 \pm 0.01$ & $16.59 \pm 0.01$ & $16.31 \pm 0.01$ \\
\hline 14 & $20.15 \pm 0.05$ & $19.00 \pm 0.01$ & $18.58 \pm 0.01$ & $18.37 \pm 0.01$ & $18.35 \pm 0.03$ \\
\hline 15 & $19.74 \pm 0.04$ & $18.34 \pm 0.01$ & $17.77 \pm 0.01$ & $17.54 \pm 0.01$ & $17.38 \pm 0.02$ \\
\hline 16 & $19.51 \pm 0.03$ & $18.52 \pm 0.01$ & $18.11 \pm 0.01$ & $17.92 \pm 0.01$ & $17.84 \pm 0.02$ \\
\hline 17 & $17.88 \pm 0.01$ & $16.54 \pm 0.01$ & $16.06 \pm 0.01$ & $15.76 \pm 0.01$ & $15.79 \pm 0.01$ \\
\hline 18 & - & $20.97 \pm 0.03$ & $19.62 \pm 0.02$ & $18.94 \pm 0.01$ & $18.68 \pm 0.04$ \\
\hline 19 & $18.65 \pm 0.02$ & $17.54 \pm 0.01$ & $17.13 \pm 0.01$ & $16.97 \pm 0.01$ & $16.88 \pm 0.01$ \\
\hline 20 & $20.21 \pm 0.05$ & $18.95 \pm 0.01$ & $18.54 \pm 0.01$ & $18.37 \pm 0.01$ & $18.33 \pm 0.03$ \\
\hline Star & $U$ & $B$ & $V$ & $R_{C}$ & $I_{C}$ \\
\hline 1 & $17.65 \pm 0.04$ & $17.49 \pm 0.03$ & $16.74 \pm 0.02$ & $16.30 \pm 0.03$ & $15.89 \pm 0.02$ \\
\hline 2 & $18.16 \pm 0.04$ & $17.89 \pm 0.03$ & $17.14 \pm 0.02$ & $16.70 \pm 0.03$ & $16.29 \pm 0.02$ \\
\hline 3 & $21.41 \pm 0.12$ & $19.95 \pm 0.03$ & $19.08 \pm 0.02$ & $18.58 \pm 0.03$ & $18.11 \pm 0.03$ \\
\hline 4 & $16.56 \pm 0.04$ & $16.24 \pm 0.03$ & $15.49 \pm 0.02$ & $15.07 \pm 0.03$ & $14.68 \pm 0.02$ \\
\hline 5 & $20.30 \pm 0.10$ & $19.81 \pm 0.03$ & $18.98 \pm 0.02$ & $18.49 \pm 0.03$ & $18.03 \pm 0.03$ \\
\hline 7 & $20.93 \pm 0.16$ & $19.87 \pm 0.03$ & $18.36 \pm 0.02$ & $17.54 \pm 0.03$ & $16.77 \pm 0.02$ \\
\hline 8 & $21.29 \pm 0.21$ & $20.69 \pm 0.04$ & $19.67 \pm 0.02$ & $19.05 \pm 0.03$ & $18.47 \pm 0.04$ \\
\hline 9 & $21.31 \pm 0.11$ & $20.21 \pm 0.04$ & $19.67 \pm 0.02$ & $19.22 \pm 0.04$ & $18.80 \pm 0.07$ \\
\hline 12 & $21.04 \pm 0.17$ & $19.76 \pm 0.03$ & $18.18 \pm 0.02$ & $17.19 \pm 0.03$ & $16.27 \pm 0.02$ \\
\hline 13 & $20.08 \pm 0.09$ & $19.00 \pm 0.03$ & $17.57 \pm 0.02$ & $16.83 \pm 0.03$ & $16.14 \pm 0.02$ \\
\hline 14 & $19.39 \pm 0.06$ & $19.37 \pm 0.03$ & $18.74 \pm 0.02$ & $18.29 \pm 0.03$ & $17.87 \pm 0.03$ \\
\hline 15 & $18.98 \pm 0.06$ & $18.77 \pm 0.03$ & $17.99 \pm 0.02$ & $17.24 \pm 0.03$ & $17.08 \pm 0.02$ \\
\hline 16 & $18.74 \pm 0.05$ & $18.86 \pm 0.02$ & $18.27 \pm 0.02$ & $17.88 \pm 0.02$ & $17.48 \pm 0.02$ \\
\hline 17 & $17.10 \pm 0.04$ & $16.94 \pm 0.03$ & $16.25 \pm 0.02$ & $15.70 \pm 0.03$ & $15.19 \pm 0.02$ \\
\hline 19 & $17.85 \pm 0.04$ & $17.88 \pm 0.03$ & $17.29 \pm 0.02$ & $16.93 \pm 0.03$ & $16.56 \pm 0.02$ \\
\hline 20 & $19.42 \pm 0.06$ & $19.32 \pm 0.02$ & $18.70 \pm 0.02$ & $18.29 \pm 0.02$ & $17.91 \pm 0.03$ \\
\hline
\end{tabular}

Notes.The transformation from Sloan to Johnson/Cousins was done using the Jester et al. (2005) transformations.

Table A.3. Host galaxy metallicities obtained with different calibrators (top part of each epoch) and a subsample of those converted to a base metallicitiy according to Kewley \& Dopita (bottom part of each epoch).

\begin{tabular}{|c|c|c|c|c|c|}
\hline Calibrator & Lines (Support lines) & $\begin{array}{c}Z_{\mathrm{Flux} 1} \\
(12+\log (\mathrm{O} / \mathrm{H}))\end{array}$ & $\begin{array}{c}Z_{\mathrm{Flux2}} \\
(12+\log (\mathrm{O} / \mathrm{H}))\end{array}$ & $\begin{array}{c}Z_{\mathrm{F} 1+\mathrm{F} 2} \\
(12+\log (\mathrm{O} / \mathrm{H}))\end{array}$ & $\begin{array}{c}Z_{\mathrm{FComb}} \\
(12+\log (\mathrm{O} / \mathrm{H}))\end{array}$ \\
\hline \multicolumn{6}{|c|}{ EPOCH 1} \\
\hline $\mathrm{D} 02^{1}$ & $\mathrm{~N} 2$ & $8.401-0.277+0.162$ & $8.409-0.276+0.174$ & $8.370-0.266+0.168$ & $8.264-0.243+0.162$ \\
\hline $\mathrm{Z} 94^{2}$ & $\mathrm{R}_{23}$ & $8.497-0.033+0.076$ & $8.688-0.015+0.028$ & $8.621-0.016+0.025$ & $8.604-0.006+0.008$ \\
\hline M91 ${ }^{3}$ & $\mathrm{R}_{23}, \mathrm{O} 3 \mathrm{O} 2(\mathrm{~N} 2, \mathrm{~N} 2 \mathrm{O} 2)$ & $8.266-0.046+0.256$ & $8.614-0.633+0.035$ & $8.553-0.491+0.015$ & $8.105-0.013+0.010$ \\
\hline $\mathrm{PP} 04 \mathrm{~N}_{2} \mathrm{Ha}^{4}$ & $\mathrm{~N} 2$ & $8.296-0.140+0.098$ & $8.300-0.138+0.100$ & $8.280-0.130+0.090$ & $8.209-0.102+0.061$ \\
\hline $\mathrm{PP} 04 \mathrm{O} 3 \mathrm{~N}^{4}$ & $\mathrm{~N} 2, \mathrm{O} 3 / \mathrm{H} \beta$ & $8.259-0.115+0.058$ & $8.263-0.108+0.058$ & $8.246-0.108+0.056$ & $8.193-0.090+0.047$ \\
\hline $\mathrm{P} 10 \mathrm{ONS}^{5}$ & {$\left[\mathrm{~N}_{\text {II }}\right] \lambda 6584 / \mathrm{H} \beta, \mathrm{O} 3 / \mathrm{H} \beta$} & $8.452-0.154+0.085$ & $8.540-0.159+0.094$ & $8.514-0.159+0.090$ & $8.408-0.113+0.072$ \\
\hline $\mathrm{P} 10 \mathrm{ON}^{5}$ & $\begin{array}{c}{[\mathrm{O} \text { II }] \lambda 3727 / \mathrm{H} \beta,\left[\mathrm{S}_{\text {II }}\right] / \mathrm{H} \beta,} \\
\mathrm{N} 2, \mathrm{O} 3 / \mathrm{H} \beta,\left[\mathrm{O}_{\text {II }}\right] \lambda 3727 / \mathrm{H} \beta\end{array}$ & $7.977-0.374+0.210$ & $8.071-0.387+0.230$ & $8.004-0.386+0.219$ & $7.707-0.287+0.186$ \\
\hline
\end{tabular}

Notes. The final metallicity applied for each region (bottom line of the table) was derived from the mean of the recalibrated metallicities in the bottom part of the third epoch. ${ }^{(*)}$ This method chooses the optimal among given: M91, KD02 N2O2, KD02 N2Ha, KD04 R23, [N2, N2O2] diagnostics (Kewley \& Ellison 2008).

References. ( ${ }^{(1)}$ Denicoló et al. (2002), (2) Zaritsky et al. (1994), ${ }^{(3)}$ McGaugh (1991), ${ }^{(4)}$ Pettini \& Pagel (2004), (5) Pilyugin et al. (2010), ${ }^{(6)}$ Maiolino et al. (2008), ${ }^{(7)}$ Marino et al. (2013), ${ }^{(8)}$ Kewley \& Dopita (2002), ${ }^{(9)}$ Kobulnicky \& Kewley (2004), Kewley \& Ellison (2008). 
Table A.3. continued.

\begin{tabular}{|c|c|c|c|c|c|}
\hline Calibrator & Lines (Support lines) & $\begin{array}{c}Z_{\text {Flux } 1} \\
(12+\log (\mathrm{O} / \mathrm{H}))\end{array}$ & $\begin{array}{c}Z_{\text {Flux2 }} \\
(12+\log (\mathrm{O} / \mathrm{H}))\end{array}$ & $\begin{array}{c}Z_{\mathrm{F} 1+\mathrm{F} 2} \\
(12+\log (\mathrm{O} / \mathrm{H}))\end{array}$ & $\begin{array}{c}Z_{\mathrm{FComb}} \\
(12+\log (\mathrm{O} / \mathrm{H}))\end{array}$ \\
\hline $\mathrm{M} 08 \mathrm{~N}^{2} \mathrm{Ha}^{6}$ & $\mathrm{~N} 2$ & $8.597-0.273+0.093$ & $8.591-0.259+0.099$ & $8.539-0.266+0.151$ & $8.386-0.226+0.117$ \\
\hline $\mathrm{M} 08 \mathrm{O}^{2} \mathrm{O}^{6}$ & $\mathrm{O} 3 \mathrm{O} 2$ & $709-0.059+0.027$ & $8.575-0.042+0.021$ & $8.615-0.031+0.019$ & $8.626-0.014+0.010$ \\
\hline $\mathrm{M} 13 \mathrm{O} 3 \mathrm{~N} 2^{7}$ & {$[\mathrm{~N}$ II $] \lambda 6584 / \mathrm{H} \beta, \mathrm{O} 3 / \mathrm{H} \beta$} & $8.219-0.077+0.039$ & $8.220-0.074+0.039$ & $8.208-0.073+0.039$ & $8.175-0.060+0.032$ \\
\hline $\mathrm{M} 13 \mathrm{~N} 2^{7}$ & {$\left[\mathrm{~N}_{\text {II }}\right] \lambda 6584 / \mathrm{H} \beta$} & $8.292-0.160+0.089$ & $8.298-0.165+0.089$ & $8.276-0.161+0.087$ & $8.201-0.131+0.077$ \\
\hline $\mathrm{KD} 02 \mathrm{~N} 2 \mathrm{O} 2^{8}$ & $\mathrm{~N} 2 \mathrm{O} 2$ & $.389-0.747+0.095$ & $8.579-0.194+0.090$ & $8.487-0.200+0.096$ & $8.293-0.733+0.109$ \\
\hline KK04 N2Ha9 & $\mathrm{N} 2, \mathrm{q},(\mathrm{N} 2 \mathrm{O} 2)$ & $616-0.182+0.099$ & $8.687-0.224+0.126$ & $8.625-0.194+0.118$ & $8.478-0.140+0.097$ \\
\hline KK04 R239 & $\mathrm{R}_{23}, \mathrm{q},(\mathrm{N} 2, \mathrm{~N} 2 \mathrm{O} 2)$ & $438-0.040+0.217$ & $8.759-0.573+0.035$ & $8.691-0.433+0.016$ & $8.288-0.010+0.008$ \\
\hline $\mathrm{KD} 2 \mathrm{comb}^{9}$ & COMBINED* & $342-0.039+0.130$ & $8.554-0.474+0.111$ & $8.450-0.292+0.126$ & $8.196-0.011+0.009$ \\
\hline KK04 & $\mathrm{R}_{23}, \mathrm{q},(\mathrm{N} 2, \mathrm{~N}$ & $326-0.038+0.208$ & $8.657-0.628+0.038$ & $8.583-0.464+0.017$ & $8.188-0.0$ \\
\hline Z94 & $\mathrm{R}_{23}$ & $3+0.098$ & $8.659-0.015+0.028$ & $590-0.017+0.027$ & $8.572-0.007+0.009$ \\
\hline M91 & $\mathrm{R}_{23}, \mathrm{O} 3 \mathrm{O} 2(\mathrm{~N} 2, \mathrm{~N} 2 \mathrm{O} 2)$ & $737-0.197+1.098$ & $8.682-0.957+0.053$ & $8.580-0.903+0.028$ & $6.889-0.082+0.063$ \\
\hline D02 & $\mathrm{N} 2$ & $551-0.234+0.137$ & $8.558-0.236+0.149$ & $8.526-0.214+0.135$ & $8.448-0.162+0.108$ \\
\hline PP04O3N & $2, \mathrm{O} 3 / \mathrm{H} \beta$ & +0.051 & $5+0.051$ & & \\
\hline & & +0.106 & $8.566-0$ & & $8.475-0$ \\
\hline \multicolumn{6}{|c|}{ EPOCH 2} \\
\hline & N2 & $8.367-0.311+0.162$ & $8.331-0.348+0.184$ & $8.287-0.311+0.188$ & $8.322-0.139+0.119$ \\
\hline $\mathrm{Z} 94^{2}$ & $\mathrm{R}_{23}$ & $8.478-0.032+0.076$ & $8.619-0.018+0.030$ & $8.571-0.018+0.027$ & $8.565-0.012+0.016$ \\
\hline M91 ${ }^{3}$ & $\mathrm{R}_{23}, \mathrm{O} 3 \mathrm{O} 2(\mathrm{~N} 2, \mathrm{~N} 2 \mathrm{O} 2)$ & $8.290-0.038+0.218$ & $8.556-0.498+0.045$ & $8.142-0.025+0.418$ & $8.141-0.021+0.016$ \\
\hline $\mathrm{PP} 04 \mathrm{~N} 2 \mathrm{Ha}^{4}$ & $\mathrm{~N} 2$ & $8.262-0.147+0.104$ & $8.240-0.155+0.107$ & $8.228-0.156+0.091$ & $8.238-0.035+0.026$ \\
\hline PP04 O & $\mathrm{N} 2, \mathrm{O} 3 / \mathrm{H} \beta$ & $21-0.126+0.067$ & $8.210-0.135+0.072$ & & $8.211-0.028+0.019$ \\
\hline P10 ONS 5 & $\begin{array}{r}{\left[\mathrm{N}_{\text {III }}\right] \lambda 658} \\
{[\mathrm{O} \text { II }] \lambda 3727}\end{array}$ & & & & \\
\hline $\mathrm{P} 10 \mathrm{ON}^{5}$ & & & $7.957-0.408+0.275$ & & \\
\hline M08 & $\mathrm{N} 2$ & & 49 & & \\
\hline M08 & $\mathrm{O} 3 \mathrm{O} 2$ & $3+0.027$ & 21 & & $14+0.010$ \\
\hline M13 & {$\left[\mathrm{N}_{\mathrm{II}}\right] \lambda 6584 / \mathrm{H} \beta, \mathrm{O} 3 / \mathrm{H} \beta$} & & & 45 & 015 \\
\hline M13 I & {$\left[\mathrm{N}_{\text {III }}\right] \lambda 6584 / \mathrm{H} \beta$} & 0.098 & +0.108 & $8.216-$ & 8.23 \\
\hline $\mathrm{KD} 02 \mathrm{~N}$ & $\mathrm{~N} 2 \mathrm{O} 2$ & $8.311-0.741+0.117$ & $8.455-0$. & $8.366-0.7$ & $8.319-0$. \\
\hline KK04 N & $\mathrm{N} 2, \mathrm{q},(\mathrm{N} 2 \mathrm{O} 2)$ & $617-0.146+0.099$ & $8.600-0.2$ & $8.564-0$. & $8.488-0$ \\
\hline KK04 R239 & $\mathrm{R}_{23}, \mathrm{q},(\mathrm{N} 2, \mathrm{~N} 2 \mathrm{O} 2)$ & $.465-0.034+0.165$ & $8.691-0.432+0.048$ & $8.321-0.020+0.372$ & $8.319-0$. \\
\hline $\mathrm{KD} 2 \mathrm{comb}^{9}$ & & $.359-0.025+0.022$ & $8.173-0.019+0.385$ & $8.223-0.018+0.232$ & $8.230-0.0$ \\
\hline KK04 & & $3+0.161$ & $3+0.051$ & & +0.012 \\
\hline Z94 & & & +0.032 & 31 & +0.019 \\
\hline M91 & $\mathrm{R}_{23}, \mathrm{O} 3 \mathrm{O} 2(\mathrm{~N} 2, \mathrm{~N} 2 \mathrm{O} 2)$ & $3+0.877$ & +0.082 & $7.114-0$. & $2+0.093$ \\
\hline D02 & N2 & 29 & +0.138 & & \\
\hline $\mathrm{PP} 04 \mathrm{O} 3 \mathrm{~N} 2$ & $2, \mathrm{O} 3 / \mathrm{H} \beta$ & +0.057 & -0.061 & & +0.016 \\
\hline & $\mathrm{N} 2$ & $26-0.151+0.107$ & $8.504-0$ & 887 & +0.025 \\
\hline \multicolumn{6}{|c|}{ EPOCH 3} \\
\hline $\mathrm{D} 02^{1}$ & N2 & $8.529-0.197+0.129$ & $8.557-0.138+0.106$ & $8.545-0.134+0.108$ & $8.408-0.123+0.115$ \\
\hline $\mathrm{Z}^{2} 4^{2}$ & $\mathrm{R}_{23}$ & $.488-0.035+0.081$ & $8.621-0.017+0.030$ & $8.578-0.017+0.028$ & $8.572-0.010+0.013$ \\
\hline M91 ${ }^{3}$ & $\mathrm{R}_{23}, \mathrm{O} 3 \mathrm{O} 2(\mathrm{~N} 2, \mathrm{~N} 2 \mathrm{O} 2)$ & $8.449-0.030+0.046$ & $8.576-0.016+0.026$ & $8.539-0.015+0.023$ & $8.532-0.009+0.011$ \\
\hline $\mathrm{PP} 04 \mathrm{~N}_{2} \mathrm{Ha}^{4}$ & $\mathrm{~N} 2$ & $.392-0.124+0.072$ & $8.414-0.091+0.057$ & $8.405-0.076+0.049$ & $8.294-0.031+0.024$ \\
\hline & & & & & +0.016 \\
\hline & $\begin{array}{c}{\left[\mathrm{N}_{\mathrm{II}}\right] \lambda 6584 / \mathrm{H} \beta, \mathrm{O} 3 / \mathrm{H} \beta} \\
{\left[\mathrm{O}_{\mathrm{II}}\right] \lambda 3727 / \mathrm{H} \beta,\left[\mathrm{S}_{\mathrm{II}}\right] / \mathrm{H} \beta,}\end{array}$ & & & & $8.635-0.025+0.017$ \\
\hline & $2, \mathrm{O} 3 / \mathrm{H} \beta,\left[\mathrm{O}_{\text {II }}\right] \lambda 3727 / \mathrm{H} \beta$ & $51+0.103$ & $8.590-0.17$ & $8.534-0.145+0$. & $8.177-0.073+0.050$ \\
\hline $\mathrm{M} 08 \mathrm{~N}^{2} \mathrm{Ha}^{6}$ & $\mathrm{~N} 2$ & $.684-0.179+0.071$ & $8.699-0$ & $8.689-c$ & +0.038 \\
\hline $\mathrm{M} 08 \mathrm{O} 3 \mathrm{O} 2^{6}$ & $\mathrm{O} 3 \mathrm{O} 2$ & $.688-0.060+0.024$ & $8.565-0$. & $30+0.017$ & $8.623-0$ \\
\hline $\mathrm{M} 13 \mathrm{O} 3 \mathrm{~N} 2^{7}$ & {$\left[\mathrm{~N}_{\mathrm{II}}\right] \lambda 6584 / \mathrm{H} \beta, \mathrm{O} 3 / \mathrm{H} \beta$} & $.251-0.051+0.024$ & $8.256-0$. & $29+0.016$ & $8.213-0$. \\
\hline M13 N2 $2^{7}$ & {$\left[\mathrm{~N}_{\text {III }}\right] \lambda 6584 / \mathrm{H} \beta$} & $.371-0.110+0.059$ & $8.388-0.078+0.051$ & $8.381-0.065+0.050$ & $8.292-0.044+0.043$ \\
\hline $\mathrm{KD} 02 \mathrm{~N} 2 \mathrm{O} 2^{8}$ & $\mathrm{~N} 2 \mathrm{O} 2$ & $8.600-0.089+0.033$ & $8.734-0.049+0.022$ & $8.689-0.045+0.021$ & $8.522-0.032+0.021$ \\
\hline KK04 N2Ha & $\mathrm{N} 2, \mathrm{q},(\mathrm{N} 2 \mathrm{O} 2)$ & $8.746-0.152+0.064$ & $8.856-0.111+0.052$ & $8.813-0.094+0.046$ & $8.643-0.063+0.045$ \\
\hline KK04 R239 & $\mathrm{R}_{23}, \mathrm{q},(\mathrm{N} 2, \mathrm{~N} 2 \mathrm{O} 2)$ & $8.569-0.035+0.052$ & $8.711-0.017+0.027$ & $8.671-0.017+0.025$ & $8.662-0.010+0.013$ \\
\hline KD02 comb $^{9}$ & COMBINED* & $.598-0.109+0.035$ & $8.734-0.051+0.022$ & $8.689-0.046+0.021$ & $8.522-0.032+0.021$ \\
\hline KK04 & $\mathrm{R}_{23}, \mathrm{q},(\mathrm{N} 2, \mathrm{~N} 2 \mathrm{O} 2)$ & & & & $8.553-0.011+0.014$ \\
\hline Z94 & $\mathrm{R}_{23}$ & $8.432-0.046+0.106$ & $8.590-0.018+0.032$ & $8.542-0.019+0.032$ & $8.536-0.012+0.015$ \\
\hline M91 & $\mathrm{R}_{23}, \mathrm{O} 3 \mathrm{O} 2(\mathrm{~N} 2, \mathrm{~N} 2 \mathrm{O} 2)$ & $8.354-0.077+0.117$ & $8.620-0.027+0.044$ & $8.553-0.029+0.044$ & $8.540-0.018+0.022$ \\
\hline D02 & $\mathrm{N} 2$ & $8.670-0.199+0.130$ & $8.699-0.144+0.111$ & $8.686-0.138+0.111$ & $8.557-0.105+0.098$ \\
\hline PP04O3N2 & $\mathrm{N} 2, \mathrm{O} 3 / \mathrm{H} \beta$ & $.577-0.069+0.031$ & $8.583-0.046+0.024$ & $8.579-0.038+0.021$ & $8.525-0.019+0.014$ \\
\hline PP04N2Ha & $\mathrm{N} 2$ & $8.672-0.146+0.085$ & $8.697-0.108+0.067$ & $8.687-0.090+0.058$ & $8.560-0.033+0.026$ \\
\hline Mean EPOCH 3 & & $8.527-0.133+0.135$ & $8.632-0.054+0.054$ & $8.602-0.068+0.068$ & $8.545-0.015+0.016$ \\
\hline
\end{tabular}

